Aus der Abteilung Allgemeinmedizin

(Prof. Dr. med. M. M. Kochen, MPH, FRCGP)

im Zentrum Innere Medizin

der Medizinischen Fakultät der Universität Göttingen

\title{
Epidemiologie und Behandlung von Kreuzschmerzen in der Hausarztpraxis
}

\author{
INAUGURAL - DISSERTATION \\ zur Erlangung des Doktorgrades \\ der Medizinischen Fakultät \\ der Georg-August-Universität zu Göttingen
}

vorgelegt von

Katja Kögel

aus

Annaberg-Buchholz

Göttingen 2007 
De k a n:

Prof. Dr. med. C. Frömmel

I. Berichterstatter: $\quad$ Prof. Dr. med. M. M. Kochen, MPH, FRCGP

II. Berichterstatterlin:

III. Berichterstatterlin:

Tag der mündlichen Prüfung: 
Inhaltsverzeichnis

Seite

$\begin{array}{ll}\text { 1. } & \text { Einleitung }\end{array}$

2. Stand der Forschung 10

2.1 Definition 10

2.2 Epidemiologie 11

2.3 Klassifikation, Symptome und mögliche Ursachen 14

2.3.1 Akute unkomplizierte Kreuzschmerzen 15

2.3.2 Radikuläre Kreuzschmerzen 15

2.3.3 Komplizierte Kreuzschmerzen und abwendbar gefährliche Verläufe 16

2.3.4 Ätiologie 16

$\begin{array}{lll}2.4 & \text { Behandlung } & 17\end{array}$

$2.5 \quad$ Arzt-Patient-Beziehung 24

3. Material und Methoden 27

$\begin{array}{lll}3.1 & \text { Studiendesign } & 27\end{array}$

$\begin{array}{lll}3.2 & \text { Patientenfragebogen } & 28\end{array}$

3.3 Fragebogen zum 1. Telefoninterview 29

3.4 Fragebogen zum 2. Telefoninterview 30

3.5 Das Schmerztagebuch 31

3.6 Pilotstudie 32

3.7 Auswahl der Arztpraxen 32

3.8 Auswahl der Patienten und Ablauf der Patientenbefragung 33

$\begin{array}{lll}3.9 & \text { Auswertung } & 34\end{array}$

4. Ergebnisse $\quad 35$

4.1 Teilnahmebereitschaft an der Studie $\quad 35$

4.1.1 Ärzte 35

4.1.2 Patientenbeteiligung 36

4.2 Epidemiologie von Kreuzschmerzen in der hausärztlichen Praxis 37

$\begin{array}{lll}\text { 4.2.1 Praxisprävalenz } & 37\end{array}$

4.2.2 Punktprävalenz nach dem Follow-up 39

$\begin{array}{lll}4.2 .3 & \text { Inzidenz } & 39\end{array}$ 
$\begin{array}{lll}\text { 4.2.4 } & \text { Rezidivrate } & 39\end{array}$

4.3 Inanspruchnahme von Versorgungsleistungen 40

4.3.1 Hausärztliche Behandlung $(n=49) \quad 40$

4.3.1.1 Verschreibung von Medikamenten 41

4.3.1.2 Verabreichung von Injektionen $\quad 42$

4.3.1.3 Durchführung weiterer Behandlungen $\quad 42$

4.3.1.4 Überweisung an einen Facharzt 43

4.3.1.5 Bildgebende Verfahren 43

4.3.1.6 Ärztliche Anregungen $\quad 43$

4.3.1.7 Ausstellen einer Arbeitsunfähigkeitsbescheinung 44

4.3.1.8 Rentenantrag $\quad 45$

4.3.2 Versorgungsleistungen im Follow-up $(n=40) \quad 46$

4.3.2.1 Arztbesuch in den vergangenen 12 Wochen 46

4.3.2.2 Verschreibung von Medikamenten 46

4.3.2.3 Verabreichung von Injektionen 47

4.3.2.4 Durchführung weiterer Behandlungen 48

$\begin{array}{lll}4.4 & \text { Krankheitsverlauf } & 48\end{array}$

$\begin{array}{lll}4.4 .1 & \text { Schmerzentwicklung } & 48\end{array}$

4.4.2 Entwicklung der Funktionalität 49

4.4.3 Subjektiver Krankheitsverlauf 53

$4.5 \quad$ Patienteneinstellung 54

4.5.1 Selbstbewältigungsstrategien 54

4.5.2 Selbsthilfe, außer durch Medikamente 55

4.5.3 Beste Behandlung für die Schmerzen 55

4.5.4 Wunsch nach weiterer Diagnostik 56

4.5.5 Erwartungen des Patienten bei der Arztkonsultation und Erfüllung 56 dieser Erwartungen

4.5.6 Offene Fragen an den Arzt 59

4.5.7 Veränderungen der körperlichen Aktivität 60

$\begin{array}{lll}\text { 4.5.8 Veränderungen im Alltag } & 62\end{array}$

4.5.9 Krankheitsvorstellungen der Patienten 63

4.6 Pilotierung des Schmerztagebuchs 64

$\begin{array}{lll}\text { 4.6.1 Methodik } & 64\end{array}$

$\begin{array}{lll}\text { 4.6.2 } & \text { Ergebnisdarstellung } & 64\end{array}$ 
$\begin{array}{lll}\text { 5. Diskussion } & 68\end{array}$

$\begin{array}{lll}5.1 & \text { Hauptaussage der Studie } & 68\end{array}$

5.2 Vergleich der Studienergebnisse mit internationaler Literatur 68

$\begin{array}{lll}5.3 & \text { Beurteilung der Methode } & 72\end{array}$

$\begin{array}{lll}5.4 & \text { Ausblick und Schlussfolgerung } & 74\end{array}$

$\begin{array}{lll}\text { 6. } & \text { Zusammenfassung } & 76\end{array}$

$\begin{array}{lll}\text { 7. } & \text { Literaturverzeichnis } & 77\end{array}$

$\begin{array}{lll}\text { 8. } & \text { Abkürzungsverzeichnis } & 83\end{array}$

9. $\quad$ Anhang $\quad 84$

9.1 Handzettel für den Arzttresen 84

9.2 Zettel für die Anmeldung bzw. Strichliste für den Hausarzt 85

$9.3 \quad$ Fragebogen 86

9.4 Telefoninterview nach 2 und 12 Wochen 88

$\begin{array}{lll}9.5 & \text { Schmerztagebuch } & 90\end{array}$ 
Verzeichnis der Abbildungen

Abbildung 1: "The real course of back pain." 11

Abbildung 2: Studiendesign 28

Abbildung 3: Teilnahmebereitschaft der Arztpraxen 35

Abbildung 4: Teilnahmebereitschaft der Patienten 36

Abbildung 5: Anzahl der Kreuzschmerzpatienten in Arztpraxen nach 37

Zuordnung in Altersgruppen $(n=121)$

Abbildung 6: Punktprävalenz von Kreuzschmerzen nach 3 Monaten 39 $(n=40)$

Abbildung 7: Häufigkeit auftretender Kreuzschmerzen während des Follow-up $(n=40)$

Abbildung 8: Medikamente mit Namen der Wirkgruppe und Zahl der Verordnungen in \%

Abbildung 9: Ärztliche Anregungen ( $n=40) \quad 44$

Abbildung 10: Arbeitsunfähigkeitstage von Patienten mit Kreuzschmerzen $\quad 45$ im Vergleich zur angegebenen Schmerzintensität am Tag der Hausarztkonsultation $(n=11)$

Abbildung 11: Verschreibung von Medikamenten im Follow-up von 3 Monaten

Abbildung 12: Verbesserung der Beweglichkeit ( $n=49) \quad 50$

Abbildung 13: Vergleich der durchschnittlichen funktionellen 51 Beeinträchtigung in Tätigkeiten und Bedürfnissen zwischen dem 1. und 2. Telefoninterview (Zeitraum 3 Monate; $n=40$ )

Abbildung 14: Vergleich der Parameter „Schmerzintensität“, „Einschränkung durch die Schmerzen“ und „Wohlbefinden“ im Krankheitsverlauf von 3 Monaten $(n=40)$

Abbildung 15: Subjektive Prognose aus Patientensicht über die Weiterentwicklung der Schmerzen $(n=49)$

Abbildung 16: Selbstbewältigungsversuche der Patienten ( $n=127)$, um Schmerzen zu lindern (276 Angaben insgesamt)

Abbildung 17: Beste Behandlung für die Beschwerden aus Patientensicht 56 $(n=76)$ 
Abbildung 18: „Was ist für Sie bei Ihrem heutigen Hausarztbesuch am Wichtigsten?" ( $n=110)$

Abbildung 19: Häufig gestellte Fragen an den Hausarzt mit Originalzitaten 59 von Patienten $(n=50)$

Abbildung 20: Veränderungen in der Aktivität in den letzten 12 Wochen $(n=21)$

Abbildung 21: „Was haben Sie selbst in den vergangenen 3 Monaten gegen Ihre Kreuzschmerzen unternommen (Veränderungen im Alltag)?" $(\mathrm{n}=17)$

Abbildung 22: Ursache der Kreuzschmerzen aus Patientensicht ( $n=49)$ 63

Abbildung 23: Vergleich der Daten für „Schmerzintensität“, „Einschränkung durch die Schmerzen“, „Einflussnahme auf die Schmerzen“ und „Wohlbefinden“ 2 Wochen (1. Telefoninterview) und 14 Wochen (Schmerztagebuch) nach der Arztkonsultation $(n=26)$

Verzeichnis der Tabellen

Tabelle 1: Praxenübersicht mit Angabe der mittleren 1-Wochen-Praxisprävalenz

Tabelle 2: Gabe von Injektionen bei der Hausarztkonsultation $(n=49)$

Tabelle 3: Durchführung weiterer Behandlungen am Tag der Hausarztkonsultation $(n=49)$

Tabelle 4: Gabe von Injektionen beim Arztbesuch in den letzten 12 Wochen $(n=40)$

Tabelle 5: Durchführung weiterer Behandlungen am Tag der Hausarztkonsultation

Tabelle 6: Kumulative Häufigkeiten der Angaben zur Schmerzstärke im Follow-up

Tabelle 7: Beeinträchtigung in Tätigkeiten und Bedürfnissen (n=49 bzw. 40)

Tabelle 8: Wohlbefinden ( $n=49$ bzw. 40) 
Tabelle 9: Selbsthilfe, außer durch Medikamente („Wie gut konnten

Sie heute, außer durch Medikamente, Einfluss auf Ihre

Schmerzen nehmen?") ( $n=49)$

Tabelle 10: Erfüllung der Erwartung an den Hausarztbesuch

Tabelle 11: Empfehlungen der Ärzte zum Zeitpunkt der Rekrutierung $(n=40)$

Tabelle 12: Vergleich, inwieweit die Patienten, denen „Bewegung“ empfohlen wurde, den ärztlichen Rat annahmen $(n=25)$

Tabelle 13: Übersicht der Durchschnittswerte von 4 Items aus dem 65 Schmerztagebuch $(n=26)$ 


\section{Einleitung}

„Rückenschmerzen“ und „Kreuzschmerzen“ (KS) werden häufig synonym verwendet, wobei mit dem Kreuzschmerz in der Regel eher der tiefe Rückenschmerz gemeint ist. Rückenschmerzen stellen überwiegend ein in komplexere Schmerzsyndrome eingebundenes unspezifisches Beschwerdebild dar. Der Kreuzschmerz ist ein Symptomenkomplex, der nur in seltenen Fällen auf greifbare Ursachen wie Bandscheibenvorfall, maligne Tumoren, viszerale, neurogene oder vaskuläre Störungen zurückzuführen ist.

KS und Rückenschmerzen sind in der Bevölkerung weit verbreitet. Sie stellen besonders in Industrienationen ein signifikantes Gesundheitsproblem dar. 80 - 90\% der Bewohner in Industriestaaten bekommen im Laufe ihres Lebens Rückenschmerzen - meist zwischen dem 50. und 64. Lebensjahr. Zur Entwicklung chronischer Schmerzen (5-10\%) können mehrere Faktoren beitragen, z.B. auch psycho-soziale Aspekte wie Stress im beruflichen oder privaten Alltag, depressive Stimmung oder ein ungünstiger Umgang mit dem Schmerz.

Die große Bedeutung von Rückenschmerzen zeigt sich an den volkwirtschaftlichen Gesamtkosten (in Deutschland rund 15-20 Milliarden Euro pro Jahr), wobei 70\% allein durch Arbeitsausfälle und Frühberentungen entstehen. In Großbritannien wurden 1993 insgesamt drei Millionen Hausarztkonsultationen für Rückenschmerzen statistisch festgehalten; zugleich ist dieses Leiden dort der häufigste Grund für körperliche Einschränkungen unter der arbeitenden Bevölkerung (Clinical Standards Advisory Group 1994).

Über den Verlauf von Rückenschmerzen, die hausärztliche Versorgung dieses Volksleidens und über die Wünsche und Erwartungen von betroffenen Patienten ist wenig bekannt.

Ziel dieser Studie ist es, etwas über die Epidemiologie von KS zu erfahren, den Krankheitsverlauf zu beobachten, die hausärztliche Behandlung zu prüfen und auch die Einstellung der Patienten bezüglich ihrer KS, z.B. Krankheitsvorstellung und Selbstbewältigungsstrategien, zu erörtern. 


\section{Stand der Forschung}

\subsection{Definition}

KS (untere Rückenschmerzen) sind Schmerzen im Bereich des Rückens vom unteren Rippenbogen bis zu den Glutäalfalten, evtl. mit Ausstrahlung in die Beine. KSen können durch unterschiedliche Ursachen (s. 2.3) ausgelöst und von verschiedenen Symptomen (s. 2.3) begleitet werden (Becker et al. 2003).

Akute KS bezeichnen Schmerzepisoden von weniger als 12 Wochen Dauer. Die Schmerzintensität kann während dieses Zeitraumes variieren. Akute KS, die länger als 6 Wochen bestehen, werden auch als „subakut“ benannt. Nachemson und Bigos (1984) definieren akute KS als plötzlich auftretend und subakute KS als Schmerzen gleicher Dauer mit langsamem Beginn. Rezidivierende KS sind akute KS, die nach einem symptomfreien Intervall von mindestens 6 Monaten erneut auftreten. Sie werden als wiederkehrende Episode akuter KS betrachtet und wie diese behandelt. Chronische KS sind Schmerzen, die seit 12 Wochen und länger bestehen. Sie können während dieser Zeit in Intensität und Ausprägung variieren (Becker et al. 2003).

Diese herkömmliche klinische Einteilung ist zwar in Klinik und Forschung weit verbreitet, entspricht aber einem starren Schema und wird durch epidemiologische Daten in dieser Form nicht unterstützt. So beschreibt Waddell 1998 KS als ein eher rezidivierendes, intermittierendes und episodisches Problem und beruft sich dabei auf eine Beobachtung von Croft et al. (1997), die den Verlauf von KS grafisch definieren (s. Abbildung 1).

Die Studie „Epidemiologie und Behandlung von Kreuzschmerzen in der Hausarztpraxis" orientiert sich in ihren zugrunde liegenden Definitionen an der Leitlinie der Deutschen Gesellschaft für Allgemeinmedizin und Familienmedizin (DEGAM; Becker et al. 2003). 


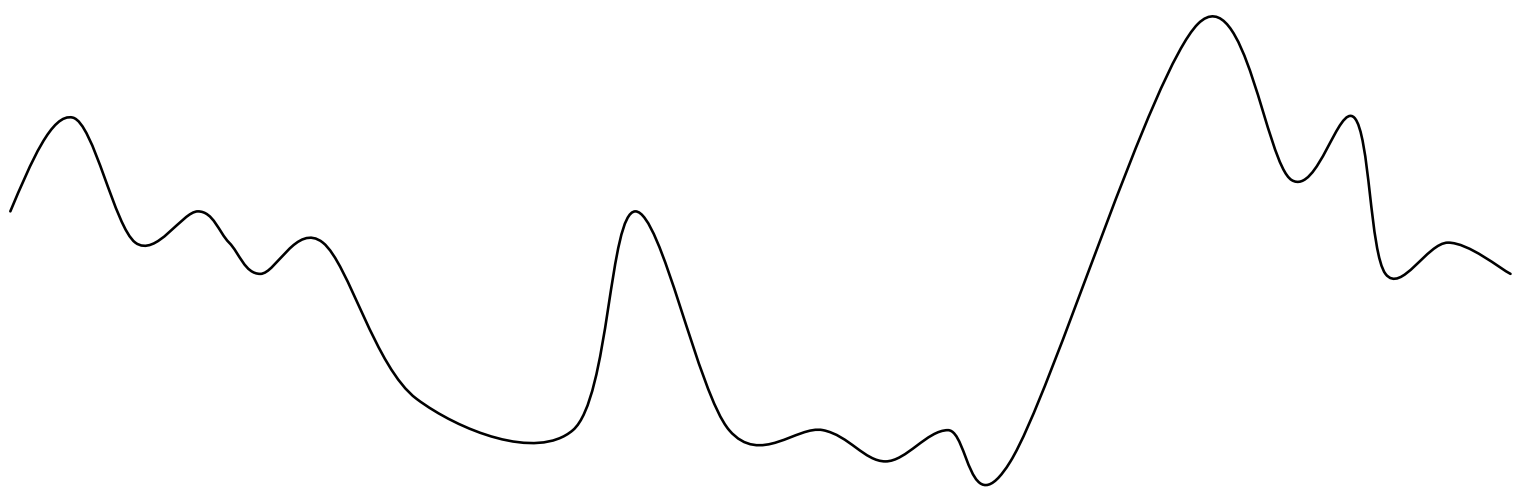

Back pain over long periods of an individual's life

Abbildung 1: Croft et al (1997): "The real course of back pain", S.73

\section{$2.2 \quad$ Epidemiologie}

Bei der Durchführung einer explorativen Analyse über KS recherchierte Freeborn Daten einer Untersuchung aus dem Jahr 1987 und fand eine jährliche Inzidenzrate von 6-7\% für KS bei Erwachsenen; 75\% dieser Kreuzschmerzepisoden wurden erstmals durch Internisten und Allgemeinärzte behandelt, wobei zwei Drittel dieser Patienten eine unspezifische Diagnose erhielten (Freeborn et al. 1997). 1998 beschrieben Croft et al. in einer prospektiven Studie eine jährliche kumulative Inzidenzrate von 6,4\% für KS unter Erwachsenen in Allgemeinarztpraxen.

Raspe und Kohlmann (1993) zeigten in einer Studie über KS die nationalen Unterschiede in England und Deutschland bezüglich der deutlich unterschiedlichen Prävalenzraten auf. Über 6000 Patienten wurden insgesamt in beiden Ländern randomisiert ausgewählt und per Fragebogen (FB) zu aktuellen KS und deren Schmerzstärke befragt. Deutsche Teilnehmer litten häufiger an aktuellen bzw. vergangenen Kreuzschmerzepisoden. Die Autoren führten diese Ergebnisse auf die interkulturellen Unterschiede in der Wahrnehmung und Beschreibung von KS zurück. 
80\% der amerikanischen Bevölkerung leiden mindestens einmal in ihrem Leben an KS. Der so genannte „low back pain“ ist in den USA einer der 10 häufigsten Gründe, weshalb Patienten ihren Hausarzt $(\mathrm{HA})$ aufsuchen und ist für ein Drittel der Gesamtkosten für Arbeitsunfähigkeit $(A U)$ und Rehabilitation verantwortlich (Henley 2000). Vergleichbare Zahlen (Raspe, Kohlmann 1993) gelten für europäische Industrienationen. $60-80 \%$ der deutschen Bevölkerung haben in ihrem Leben schon einmal KS verspürt, wobei jüngere Menschen häufiger betroffen sind als ältere. So betrugen 1990 in den USA beispielsweise die direkten Kosten für die Gesundheitsversorgung von KS über 24 Milliarden US-Dollar; die Gesamtkosten einschließlich Rehabilitation durch Behinderung, Arbeitsausfall, Produktionsverluste etc. wurden auf über 100 Milliarden US-Dollar geschätzt (Frymoyer, Kats-Baril 1991).

Frymoyer et al. (1980) sahen retrospektiv mehr als 3900 Patientenakten einer allgemeinärztlichen Praxis aus den Jahren 1975-1978 ein und prüften diese auf das Vorkommen von KS und deren Zusammenhänge mit einzelnen Umwelteinflüssen und psychischen Belastungen. Die Autoren berichten über eine Inzidenz von Schmerzen des unteren Rückens von $11 \%$ bei den Männern und 9,5\% bei den Frauen innerhalb des o.g. 3-Jahres-Intervalls. Es fand sich ein signifikanter Zusammenhang zwischen den beklagten Beschwerden und schwerer körperlicher Beanspruchung der Wirbelsäule wie Tragen, Heben, Lastwagen fahren oder Vibrationsbelastungen. Patienten mit solchen Beschwerden waren auch gehäuft von Angst- und Depressionsepisoden betroffen.

1998 führten van den Hoogen et al. eine Studie durch, mit dem Ziel, den Verlauf von $\mathrm{KS}$ in der Allgemeinarztpraxis zu untersuchen und eine Beurteilung der Prognose zu ermöglichen. Über einen Zeitraum von 2 Jahren wurden Patienten mit KS rekrutiert und deren Verlauf über 12 Monate protokolliert. $269(60,7 \%)$ von 443 Patienten wurden für den gesamten Zeitraum des Follow-up beobachtet. Obwohl die Schmerzen und die Funktionseinschränkung sich schnell nach der ersten hausärztlichen Konsultation verringerten, litten nach 3 Monaten noch immer 35\% und nach 12 Monaten 10\% der teilnehmenden Patienten unter KS. Im Durchschnitt beklagten Patienten nach 7 Wochen ein Rezidiv ihrer KS, welches im Mittel 6 Wochen dauerte (van den Hoogen et al. 1998). 
Croft et al. (1998) führten eine prospektive Studie mit Patienten durch, die sich wegen $\mathrm{KS}$ in einer Allgemeinarztpraxis vorstellten und im Follow-up nach 1-2 Wochen, 3 und 12 Monaten interviewt wurden. Nach 2 Wochen waren nur 5\% komplett beschwerdefrei, nach 3 Monaten 21\% und nach 12 Monaten 25\%. Durchschnittlich litten die Patienten 3 Wochen an KS. Die meisten Patienten mit KS stellten sich in den ersten 3 Monaten nach der primären Konsultation nicht erneut bei ihrem HA vor; nur 8\% setzten ihre Arztbesuche auch nach Ablauf von 3 Monaten fort.

Internationale Studien (Croft et al. 1994) erhärten mit ihren Ergebnissen nicht nur den Aspekt der hohen Lebenszeitprävalenz (60\%), sondern zeigen auch hohe Punkt-, Monats- und Jahresprävalenzen. 1998 schrieb Gordon Waddell, ein bekannter Orthopäde, der sich seit über 20 Jahren mit dem Thema Rückenschmerzen auseinandersetzt, das Buch „The back pain revolution“. Hierin beschreibt er systematisch die Geschichte des Rückenschmerzes bis hin zur Entwicklung eines neuen klinischen Modells für die Behandlung von KS. So zählt Mason (1994) eine Punktprävalenz für KS von 14\% auf. In der South Manchester Studie finden Papageorgiou et al. (1995) eine Monatsprävalenz von 39\%. Walsh et al. (1992) und Mason (1994) berichten von einer Jahresprävalenz von 36-37\%. Verschiedene internationale Studien belegen Lebenszeitprävalenzen von 58-59\% (Skovron et al. 1994; Walsh et al. 1992; Papageorgiou et al. 1995) bzw. 60-80\% steigend mit dem Alter (Biering-Soerensen 1983), mit einer Häufung zwischen dem 50. und 64. Lebensjahr (Schochat, Jackel 1998).

Die epidemiologische Forschung um das Thema Rückenschmerzen begann in Deutschland Ende der 80er Jahre. Hier sind Studien der Arbeitsgruppe um Raspe mit Befragungen in Lübeck, Hannover und Bad Säckingen hervorzuheben. In einer großen Studie von 1984-87 wurden aus dem Einwohnermelderegister der Stadt Hannover repräsentative systematische Zufallsstichproben von über 5000 Einwohnern im Alter zwischen 25 und 74 Jahren gezogen (Raspe et al. 1990). Frauen zeigen sich hier in jeder Altersgruppe stärker durch Rückenschmerzen belastet als Männer. Die Rückenschmerzbelastung aller Antwortenden steigt mit zunehmendem Alter an. Mehr als 10\% aller Arbeitsunfähigkeitsfälle und mehr als ein Drittel aller medizinischen Rehabilitationen entfallen auf Rückenschmerzen. Raspe macht am Beispiel der Bundesrepublik den epidemiologischen Trend von 
Rückenschmerzen in den Jahren zwischen 1983 und 1990 deutlich: es zeigt sich sowohl eine Zunahme der Zahl und relativen Häufigkeit der Arbeitsunfähigkeitsfälle (Männer 37\%, Frauen 13\%) als auch ein Zuwachs bei der Inanspruchnahme von so genannten Heilverfahren wie stationäre Rehabilitation durch die gesetzliche Rentenversicherung. Hingegen zeigen die Zahlen der Krankenhausfälle und vorzeitigen Berentungen wegen Rückenerkrankungen, also sogenannte höher eingestufte teurere Leistungen, einen Rückgang (Raspe, Kohlmann 1994). In einer Querschnittserhebung wurden über 1000 Patienten in einer allgemeinärztlichen Praxis in gemischt städtisch-ländlichem Gebiet innerhalb eines Quartals 1998 zum Thema Rückenschmerzen befragt. $60 \%$ der Patienten, die wegen aktueller Rückenschmerzen in die Praxis kamen, hatten Beschwerden des unteren Rückens (Stahmann 2001).

Diese Statistiken zeigen die Bedeutung des Themas KS für den einzelnen Patienten, für den Arzt in seiner täglichen Arbeit, aber auch für die gesamte Volkswirtschaft.

\subsection{Klassifikation, Symptome und mögliche Ursachen}

KS sind häufig und in ihrem Erscheinungsbild meist inhomogen. Sie können von einer Vielzahl anatomischer Strukturen ausgehen, doch bleibt trotz Diagnostik bei ca. 85\% der Patienten die genaue Ursache der Beschwerden unklar (White, Gordon 1982). In 80-90\% der Fälle erholen sich die Patienten von ihren Schmerzen innerhalb von 4 bis 6 Wochen, unabhängig von der Art und Ausübung der Behandlung (Waddell 1987). An erster Stelle steht deshalb nicht die Ursachenforschung, sondern das Erkennen von abwendbar gefährlichen Verläufen, die sofortiger Intervention bzw. weiterer Untersuchungen bedürfen. Zu wenig Diagnostik birgt die Gefahr in sich, dass eine gefährliche Erkrankung nicht erkannt wird; zu viel Diagnostik verursacht Kosten, belastet den Patienten und kann sogar zu überflüssigen und riskanten Therapien führen. So kann durch intensive Diagnostik und passive Maßnahmen das Krankheitserleben des Patienten verstärkt und über eine „iatrogene Somatisierung“ einer frühzeitigen Chronifizierung Vorschub geleistet werden (Raspe et al. 1994). Trotz der hohen Spontanheilungsrate kommt es bei bis zu zwei Dritteln der Patienten zu erneuten Rückfällen (Pengel et al. 2003); weit mehr als die Hälfte der Betroffenen leidet nach einem Jahr trotz Wiederaufnahme ihrer Arbeit und gewohnter Tätigkeiten 
noch immer oder erneut an Schmerzen und Bewegungseinschränkungen. Bei primär vom Rücken ausgehenden muskuloskelettalen Schmerzen hat sich folgende Einteilung international bewährt (Waddell 1982):

\subsubsection{Akute unkomplizierte Kreuzschmerzen}

Synonyme: Lumbago, „Hexenschuss“, unspezifische KS, nicht radikuläre KS, unspecific low back pain

Mehr als 70\% erwachsener Patienten mit KS in primärärztlicher Behandlung leiden unter „unkomplizierten KS“ (Schers et al. 2000). Sie treten spontan auf oder auch nach körperlicher Beanspruchung, wie z.B. nach Heben schwerer Lasten. Die Patienten sind im guten Allgemeinzustand und ihre Schmerzen ändern sich typischerweise je nach Körperposition und Bewegungsablauf. Häufig (in $70 \%$ der Fälle) strahlen die KS in ein oder beide Beine aus (allerdings selten unterhalb des Knies), ohne dass eine Irritation einer Nervenwurzel besteht (pseudoradikuläre Beschwerden). Sie werden als dumpfe wenig umschriebene Beschwerden geschildert (Becker et al. 2003).

\subsubsection{Radikuläre Kreuzschmerzen}

Synonyme: Ischialgie, Lumboischialgie

Ein geringer Anteil (4-5\%) der Rückenschmerzen in der Bevölkerung (etwa 14\% in hausärztlichen Praxen (Schers et al. 2000)) entsteht durch Irritation oder Kompressionen der Nervenwurzel, z.B. in Zusammenhang mit einem Bandscheibenvorfall (zu 98\% im Bereich L4-S1), einer Spinalkanalstenose oder postoperativen Narbenbildung (Heliovaara et al. 1987). Die Ausbreitung der Schmerzen ist meist auf ein oder mehrere Dermatome beschränkt. Radikuläre KS werden als klar begrenzt und scharf einschießend beschrieben, wobei die Schmerzen im Bein schlimmer als die KS erscheinen. Sie sind häufig mit Taubheitsgefühl oder Parästhesien verbunden und lassen sich durch Nervendehnung (z.B. im Lasègue-Test) auslösen (Becker et al. 2003). 


\subsubsection{Komplizierte Kreuzschmerzen und abwendbar gefährliche Verläufe}

Etwa 1-3\% (Schers et al. 2000) aller KS primärärztlicher Patienten sind auf Tumorerkrankungen, Frakturen, Infektionen, interventionsbedürftige Deformitäten (wie z.B. Spondylolisthesis im Kindesalter) oder entzündlich rheumatische Erkrankungen zurückzuführen (Deyo, Weinstein 2001). Ihr Erscheinungsbild kann dem unkomplizierter oder seltener dem radikulärer KS ähneln. Mit dem Vorliegen einer oder mehrerer der unten aufgeführten Warnhinweise steigt die Wahrscheinlichkeit, dass die Beschwerden einen gefährlichen Verlauf nehmen, auch wenn sie je nach Merkmal trotzdem gering sein kann. Die Aussagekraft einzelner Warnhinweise ist gering (geringe Sensitivität und Spezifität); erst das Gesamtbild aller Symptome ermöglicht die Einschätzung des individuellen Risikos des Patienten (Becker et al. 2003):

\section{Komplizierte Kreuzschmerzen}

- $\quad$ Alter $<20$ Jahre $>50$ Jahre

- Zunehmender, nicht bewegungsabhängiger Schmerz oder Persistenz der Beschwerden trotz Therapie

- Schlechter Allgemeinzustand

- Bekannte Tumorerkrankung

- $\quad$ Adäquates Trauma, das eine Fraktur wahrscheinlich macht

- Intravenöser Drogenabusus

- HIV-Infektion

- Systemische Steroidmedikation oder bekannte Osteoporose

- Ausgeprägte neurologische Ausfälle

z.B. Reflexauffälligkeiten, motorische und sensible Ausfälle im Versorgungsgebiet mehrerer Nervenwurzeln oder das Cauda-EquinaSyndrom (Reithosenanästhesie, Blasen- und Mastdarmstörung)

- Hinweise auf entzündlich rheumatische Erkrankungen.

\subsection{4 Ätiologie}

KS können durch unterschiedliche Störungen im Rücken entstehen, wie z.B. Muskelverspannungen, degenerative Veränderungen, Osteoporose sowie seltene Anlagestörungen des Skeletts, Entzündungen, Tumoren, Verletzungsfolgen sowie intrathekale Neubildungen. Seltener wird der Kreuzschmerz durch strukturelle 
Veränderungen abdomineller Organe, einschließlich Gefäßerkrankungen der Aorta, zustande kommen (Breitenfelder 1988).

Breitenfelder macht darauf aufmerksam, dass in diesem Zusammenhang der Vollständigkeit halber auf die psychogene Verursachung des Kreuzschmerzes hingewiesen werden muss, bei dem ein morphologisches Substrat im pathologischanatomischen Sinne nicht gefunden werden kann. Ahrens (1990) sieht einen Zusammenhang zwischen seelischen Impulsen, Konflikten oder Haltungen und deren pathophysiologischer Umsetzung im Bereich des Kreuzes in Form von tiefsitzenden Rückenschmerzen. Ein psychogener Schmerz kann grundsätzlich jede Form des körperlichen Schmerzes in vielerlei Variationen und Lokalisationen annehmen. So führt ein psychisch induzierter Kreuzschmerz in der Regel zu einer Schonhaltung des Rückens, dadurch bedingten Funktionseinbußen und bei entsprechender Chronizität letztlich zu nicht mehr vollständig revidierbaren pathophysiologischen Abläufen und pathomorphologischen Veränderungen. So wird aus einem primär psychogenen Schmerzsyndrom ein psycho-somatisches. Hasenbring (1992) betont, dass die Chronifizierung von KS eng verknüpft mit psychischen und sozialen Prozessen abläuft. KS chronifizieren, wenn die Beschwerden über 12 Monate persistieren und Therapieversuche fehlschlagen. Um diesen Prozess frühzeitig zu erkennen und aufzuhalten, sollten im ärztlichen Gespräch besonders typische Risikofaktoren erfragt werden (Berger-Schmitt et al. 1996; Keel, Schütz-Petitjean 1996). Hervorzuheben sind ein ungünstiger bisheriger Krankheitsverlauf (anhaltende, rezidivierende Beschwerden; radikuläre Schmerzen) und psychosoziale Einflussfaktoren (geringer Bildungsstand, Unzufriedenheit im Privaten und Arbeitsalltag, Depressionen, starkes Krankheitsgefühl). Patienten mit chronischen KS haben signifikant größere Komorbiditäten und fühlen sich durch diese stärker beeinträchtigt (Buchner et al. 2007).

\subsection{Behandlung}

Ziel der Kreuzschmerztherapie ist die Schmerzkontrolle, genauer gesagt, die Heilung oder Linderung akuter und chronischer Schmerzen. Die Patienten sollten möglichst in die Lage versetzt werden, ihren täglichen Verrichtungen wieder nachzukommen. Weiteres Ziel ist die Prävention chronischer Verläufe (Becker et al. 2003). Die 
Mehrheit von Kreuzschmerzpatienten kann in der Allgemeinarztpraxis behandelt werden (Freeborn et al. 1997).

\section{Patientenberatung}

Eines der wichtigsten Bestandteile der hausärztlichen Therapie ist das individuelle Patientengespräch. Bevor eine Aussage über die mögliche Ursache, die anschließende Therapie und das Procedere gemacht werden kann, muss eine ausführliche Anamnese allem vorangestellt werden. In dieser sollten Arzt und Patient mit gezielten Fragen über die Qualität, Dauer, Häufigkeit und Ausstrahlung der Schmerzen die Vorgeschichte und die derzeitige Situation erörtern. Ziel des Gespräches ist es unter anderem, den Patienten so schnell wie möglich zur Wiederaufnahme seiner üblichen Aktivität zu bewegen (Deyo 1993), da so die Beschwerden schneller gelindert, chronische Verläufe verhindert und Arbeitsunfähigkeitszeiten reduziert werden können (Waddell et al. 1997). Der Erfolg der Behandlung ist sicherlich auch zum Teil von der derzeitigen physischen und psychischen Motivationslage des Patienten abhängig. Sowohl Prochaska und Velicer (1997) als auch Keller (1999) unterscheiden verschiedene Motivationslagen des Patienten (Absichtslosigkeit, Absichtsbildung, Vorbereitung, Handlung und Aufrechterhaltung), je nachdem, ob der Patient die Notwendigkeit von Aktivität für den Heilungsprozess nachvollziehen und für sich akzeptieren kann oder ob sein Problem die Umsetzung bzw. Einbindung und Aufrechterhaltung von Aktivität im Alltag ist. Ärztlicherseits sollten je nach Motivationslage die Aufklärung über die Bedeutung von Aktivität bzw. die Bestärkung bereits bestehender Ansätze der Verhaltensänderung den Schwerpunkt der Beratung bilden. Essentiell für die Behandlung ist in jedem Fall die aktive Einbindung des Patienten.

Im Beratungsgespräch sollten folgende Themen konkretisiert werden (Becker et al. 2003):

A) Die Harmlosigkeit und gute Prognose (hohe Spontanheilungstendenz) der Beschwerden.

Bei Patienten mit akuten KS und damit zusammenhängenden Funktionseinschränkungen verbessern sich die Beschwerden normalerweise innerhalb von vier Wochen (Pengel et al. 2003). 50\% der Patienten in allgemeinärztlicher Behandlung, die wegen KS krank geschrieben sind, 
kehren innerhalb von 8 Tagen an ihren Arbeitsplatz zurück; nach einem Jahr haben noch 2\% eine Krankschreibung (Schiottz-Christensen et al. 1999).

B) Der begründete Verzicht auf weitere Diagnostik.

Diagnostiziert der HA unkomplizierte KS, ist radiologische Diagnostik nicht nötig, da sich die typischen Symptome in der Regel spontan zurückbilden (Car, Sheikh 2003).

C) Die hohe Wahrscheinlichkeit des Wiederauftretens der Beschwerden.

Eine hohe Zahl an Patienten (45\%) in allgemeinärztlicher Behandlung hat mit wiederkehrenden Beschwerden und erneuten Rückfällen von KS zu kämpfen (Schiottz-Christensen et al. 1999), die durch Bewegungsmangel gefördert bzw. durch Ausgleichsaktivität verhindert werden können.

D) Sichere und effektive Behandlungsmethoden und die Bedeutung der Aktivität für den Heilungsprozess.

Bei der Behandlung akuter KS stehen sowohl medikamentöse als auch diverse nicht-medikamentöse Therapieoptionen zur Verfügung. Wichtig ist dabei, den Patienten zu früher Aktivierung zu motivieren und von passiven Maßnahmen abzuraten.

E) Die Option weiterer diagnostischer und therapeutischer Maßnahmen bei Persistenz der Beschwerden oder Verschlechterung.

Auch rezidivierende Beschwerden bzw. Verschlechterungen können einer effektiven Behandlung zugeführt werden.

\section{Medikamentöse Therapie}

Die medikamentöse Therapie von KS sollte mit einfachen Analgetika begonnen werden. Hier wird in der DEGAM-Leitlinie (Becker et al. 2003) Paracetamol empfohlen, auch wenn es in der direkten Anwendung bei KS nicht evaluiert ist (Wörz et al. 2000).

Die nächste Stufe bei der Therapie akuter KS ist der Einsatz von nicht-steroidalen Antirheumatika (NSAR). Ihre analgetische Wirkung beruht im Wesentlichen auf der Hemmung der Cyclooxygenase und somit der Aufhebung der prostaglandin- 
bedingten Erregbarkeitssteigerung an den Synapsen des nozizeptiven Systems (Wörz et al. 2000). In einer randomisierten kontrollierten Studie (RCT, randomized controlled trial) berichtet Henley (2000) über den Einsatz und die Wirksamkeit von NSAR (ASS, Ibuprofen, Diclofenac) bei akuten KS. Auch andere RCTs belegen einerseits den effizienten Einsatz von NSAR bei unkomplizierten KS, andrerseits jedoch auch eingeschränkte bis keine Effektivität bei Kreuzschmerzpatienten mit Ischialgie und radikulären KS mit Irritation der Nervenwurzel (Koes et al. 1997). Die typischen gastrointestinalen, allergischen und pseudoallergischen Nebenwirkungen, welche mit der Anwendungsdauer häufiger werden, limitieren die Anwendung von NSAR (Wörz et al. 2000).

Der Gebrauch von Muskelrelaxanzien in der Behandlung des unspezifischen Kreuzschmerzes wird kontrovers diskutiert. In der Kurzzeitbehandlung sind sie effektiv, gehören aber aufgrund von ungünstigen Nebenwirkungen (Bewusstseinstrübungen, Schwindel, Müdigkeit) nicht zur ersten Wahl (van Tulder et al. 2003). Sie können bei Kontraindikationen für die Anwendung von NSAR kurzfristig zum Einsatz kommen (Becker et al. 2003).

Bei sehr starken Beschwerden, die auf einfache Schmerzmittel nicht ansprechen, können auch Opioidanalgetika angewandt werden. Sie wirken im zentralen Nervensystem und hemmen dort den nozizeptiven Input (Wörz et al. 2000). Der zentrale Angriffsort erklärt damit auch die dabei auftretenden Nebenwirkungen wie Benommenheit, verminderte Reaktions- und Entscheidungsfähigkeit und auch Abhängigkeit (Becker et al. 2003). Bei Patienten mit starken chronischen KS sind jedoch Abhängigkeit und Toleranzentwicklung von untergeordneter Bedeutung, weil dafür die Lebensqualität über einen langen Zeitraum wesentlich verbessert werden kann. Im Fall von Dauerschmerzen sind Retardformen (z.B. Tramadol; Tilidin/Naloxon) zu empfehlen (Wörz et al. 2000).

Die Wirksamkeit von Antidepressiva ist von unbekannter Effektivität (Henley 2000). Trizyklische Antidepressiva sind jedoch bei chronischen KS und gleichzeitig vorliegenden depressiven Störungen von Vorteil. Wichtig sind die einschleichende Dosierung und die Einmalgabe zur Nacht aufgrund der sedierenden Wirkung (Wörz et al. 2000). 
Die Applikation von Lokalanästhetika oder Glukokortikoiden in den Epiduralraum oder die Umgebung von Spinalnerven bewies in RCTs keine überzeugende Wirksamkeit (Nelemans et al. 1999). Angesichts möglicher Nebenwirkungen (Infektionen) sollte das Verfahren in der Behandlung akuter unkomplizierter KS nicht eingesetzt werden (Becker et al. 2003).

Gänzlich abzulehnende Therapieverfahren zur Behandlung akuter und chronischer KS (Becker et al. 2003) sind die Anwendung von oralen Glukokortikoiden (multiple Glukokortikoidnebenwirkungen wie gastrointestinale Ulcera, Osteoporose etc.) und die Verabreichung intravenöser und intramuskulärer Medikamenteninjektionen (wegen der Möglichkeit von auftretenden Anaphylaxien und Abszessen).

\section{Nichtmedikamentöse Therapie}

Neben der medikamentösen Therapie existieren vielfältige nicht medikamentöse

Behandlungsmethoden unterschiedlichen Evidenzgrades. Davon sind chiropraktische Methoden und Massagen die am häufigsten angewandten in den USA (Eisenberg et al. 1998).

Patienten mit chronischen KS, die von einem Chiropraktiker behandelt werden, zeigen eine deutliche Verbesserung und Zufriedenheit nach einem Monat im Vergleich zu Patienten, die keine chiropraktische Therapie erhielten (Nyiendo et al. 2000). Gegenüber ineffektiven Therapieformen scheint die Manipulations- und Mobilisationsbehandlung sowohl für akute als auch für chronische KS wirksam, jedoch im Vergleich zu evidenzbasierten konservativen Therapien nicht überlegen zu sein (Becker et al. 2003).

Massagebehandlungen zeigen bei subakuten und chronischen KS positive Effekte (Cherkin et al. 2003), besonders in Kombination mit Übungen und gezieltem Erlernen (Furlan et al. 2000). Zu anderen physikalischen Behandlungsformen wie Hitze- und Kälteanwendungen, Kurzwellentherapie und Ultraschallanwendungen sind keine Wirksamkeitsbelege vorhanden. In Einzelfällen können sie eingesetzt werden, um Befindlichkeitsverbesserungen zu erzielen (Becker et al. 2003). Ähnliches gilt für Rückenschulen. Die Patienten werden für rückengerechtes Verhalten im Alltag geschult und zu Sport ermuntert. Die 2003 herausgegebene Leitlinie in Deutschland 
empfiehlt Rückenschulungen bei Patienten mit länger anhaltenden unkomplizierten KS, die auf Therapiemethoden erster Wahl nicht angesprochen haben (Becker et al. 2003). Allerdings ist zu wenig über die Kosteneffektivität von Rückenschulen bekannt und zudem waren bisherige Studien methodisch unzureichend und qualitativ nicht hochwertig (Heymans et al. 2004).

Ebenso kontrovers diskutiert ist die Zweckmäßigkeit von Krankengymnastik. Die so genannte Physiotherapie beinhaltet entspannende Lagerung, ggf. axiale Traktionsbehandlung, spezielle Rückenübungen, Massage angespannter Muskelgruppen und das Erlernen rückenschonenden Verhaltens im Alltag. Krankengymnastik scheint nicht effektiver $\mathrm{zu}$ sein als der Rat eines Physiotherapeuten an den Patienten, aktiv und in Bewegung zu bleiben (z.B. anschaulich mittels eines Patienten-Handbuchs) (Frost et al. 2004). In RCTs fand man widersprüchliche Ergebnisse zur Traktionsbehandlung; auch mangels qualitativer Studien und wegen häufiger methodischer Hindernisse kann hierzu keine Aussage gemacht werden (Clarke et al. 2006). Bewegungstherapie scheint einen geringfügigen Effekt auf die Schmerzreduktion und Funktionsverbesserung bei chronischen Kreuzschmerzpatienten zu haben (Hayden et al. 2005). Zu empfehlen sind krankengymnastische Übungen bei Patienten mit akuten und chronischen unkomplizierten KS (Becker et al. 2003). Eine spezielle Form der Bewegungstherapie, die so genannte „Cesar Therapie“, demonstrierte in einer randomisierten klinischen Studie eine signifikante Verbesserung der Kreuzschmerzsymptome nach 6 Monaten im Vergleich zur Durchführung standardisierter Behandlungsverfahren bei chronischen $\mathrm{KS}$ durch Allgemeinmediziner (Hildebrandt et al. 2000).

Akupunktur hat in der Therapie von akuten KS einen bislang ungeklärten Stellenwert (Cherkin et al. 2003). Bei chronischen Beschwerden ist Akupunktur kurzfristig effektiver und führt zu Schmerzerleichterung und Funktionsverbesserung (Furlan et al. 2005); so können Akupunkturbehandlungen optional neben evidenzbasierten Therapie- verfahren in Abstimmung mit dem Patienten und entsprechender Risikoabwägung eingesetzt werden (Becker et al. 2003).

Die transkutane elektrische Nervenstimulation (TENS) kann bei persistierenden Beschwerden, die trotz evidenzbasierter Therapieverfahren nicht verbesserbar sind, eingesetzt werden, jedoch in Verbindung mit aktivierenden Maßnahmen und 
Verhaltenstherapie (Becker et al. 2003). Es gibt nur begrenzte Evidenz, welche den Gebrauch von TENS als eine isolierte Intervention in der Behandlung von chronischen KS unterstützt (Khadilkar et al. 2005).

Es hat sich gezeigt, dass Verhaltenstherapie bei chronischen KS zu einer effektiveren Schmerzlinderung und Funktionsverbesserung führt (Ostelo et al. 2004). Oft werden Patienten mit langer Krankheitsdauer von depressiven Symptomen beherrscht (Waxman et al. 1998). Es empfiehlt sich eine psychologische Therapie bei Patienten mit Risikofaktoren für einen chronischen Verlauf, die mit langen Arbeitsunfähigkeitszeiten einhergehen (Becker et al. 2003).

Multimodale/interdisziplinäre Therapiekonzepte zeigten sich in der Behandlung von chronischen KS gegenüber weniger intensiven Programmen überlegen, besonders, was die Rate der Arbeitplatzwiederkehrer, die Versorgungskontakte, das Ausmaß von Schmerz und Einschränkung und die körperliche Aktivität betrifft (Bendix et al. 1995). Intensive tägliche Therapieprogramme, zusammengesetzt aus körperlichem Training, psychologischen, sozialen oder ergotherapeutischen, auf die Arbeitsplatzbedingungen ausgerichteten Maßnahmen, verbessern Schmerzen und Funktion bedeutend mehr als konventionelle Therapieverfahren (Hildebrandt et al. 2000). Programme zur Wiederherstellung der Funktion bei chronischen KS haben in mehreren Ländern Effektivität bewiesen. Schmerzintensität, Einschränkung, Häufigkeit von Depressionen und psychologischen Problemen verbesserten sich signifikant wie auch die Arbeitsfähigkeit und die Beanspruchung des Gesundheitssystems. Bislang sind derartige Konzepte in Deutschland noch nicht sehr bekannt und verbreitet und werden von den Versicherungsträgern nicht honoriert (Pfingsten, Hildebrandt 2001). Erwähnenswert sei in diesem Zusammenhang auch der Stellenwert der Rehabilitation in der Behandlung von unkomplizierten KS. Rehabilitation sollte nicht als separates, zweites Stadium nach der vollständigen Therapie angesehen werden, sondern in das klinische und berufsorientierte Management von unkomplizierten KS integriert werden (Waddell, Burton 2005). Es wird empfohlen, verstärkt wohnortnahe, ambulante Rehabilitationsmaßnahmen anzubieten, die eine (teilweise) Fortsetzung der Berufsoder Alltagstätigkeit erlaubt (Sachverständigenrat zur Begutachtung der Entwicklung im Gesundheitswesen 2002). 
Bettruhe ist zur Behandlung akuter unspezifischer KS abzulehnen (Hagen et al. 2005). Bettruhe kann den Krankheitsverlauf negativ beeinflussen, z.B. chronische Verläufe forcieren und die Rehabilitation verzögern (Becker et al. 2003). Im Gegenteil, die frühe Wiederaufnahme der normalen täglichen Verrichtungen und die gesteigerte körperliche Aktivität stehen im Vordergrund (Keen et al. 1999). Aktive Bewegung bringt nicht nur kurzfristig Schmerzentlastung, sondern verbessert auch auf lange Sicht die Funktion bei akuten KS (van Tulder et al. 2006).

In der hausärztlichen Konsultation stehen folglich die exakte Anamneseerhebung und klinische Untersuchung im Vordergrund, um gefährliche Verläufe frühzeitig zu erkennen und möglichst auszuschließen. Unkomplizierte $\mathrm{KS}$ sollten keiner zusätzlichen bildgebenden Diagnostik unterzogen werden, denn dies kann dazu führen, dass Arzt und Patient sich auf nicht therapierelevante Zufallsbefunde fixieren, die nicht im Zusammenhang mit den aktuellen Beschwerden stehen (Becker et al. 2003).

\section{$2.5 \quad$ Arzt-Patient-Beziehung}

Die Entwicklung einer guten Arzt-Patienten-Beziehung ist in der hausärztlichen Praxis von nicht zu unterschätzender Bedeutung (Southgate, Bass 1983).

Schlemmer (1990) resümiert, dass Vertrauen die Basis des Verhältnisses von Arzt und Patient ist. Es ist die Voraussetzung für eine sinnvolle Zusammenarbeit beider Partner, die dem Ziel dient, Gesundheit zu erhalten oder - soweit dies möglich ist wiederherzustellen.

Schlemmer (1990) fasst zusammen, dass das ärztliche Gespräch als vertrauensbildende Maßnahme bzw. als wichtigste Voraussetzung jeglicher therapeutischer Wirkung im Verhältnis von Arzt und Patient eine entscheidende Rolle spielt. Mitmenschlichkeit ist, was sich der Patient von seinem Arzt erhofft. Im Dialog zwischen Arzt und Patient sollte auch Wert auf Prävention gelegt werden:

1. Primärpräventive Maßnahmen (sportliche Betätigung) sowie multimodale Ansätze wie betriebliche Gesundheitsförderung richten sich an gesunde Personen mit dem Ziel, das Auftreten von Rückenschmerzen zu verhindern. 
2. Sekundärpräventive Maßnahmen richten sich an Personen mit bestehenden bzw. episodisch-rezidivierenden Rückenschmerzen, mit dem Ziel, bestehende Schmerzen zu reduzieren bzw. Rezidive zu vermeiden.

3. Tertiärpräventive Maßnahmen richten sich an Rückenkranke mit chronischen bzw. chronifizierenden Beschwerden mit dem Ziel, eine Verschlechterung des Gesundheitszustandes zu vermeiden bzw. rückgängig zu machen und die Funktionsfähigkeit in Alltag und Beruf zu sichern.

McKinley und Middleton (1999) beschreiben in einem RCT die Ergebnisse über die Erwartungen einer großen Anzahl von Patienten, die ihren HA aufsuchten wie folgt: die meisten Patienten kommen mit einer bestimmten Agenda zur Hausarztkonsultation, mit Hilfe derer sie ihre offenen Fragen, Vorstellungen und Erwartungen ausdrücken. Für ein gutes Ergebnis der gemeinsamen Besprechung ist es für die Allgemeinmediziner unumgänglich, sich dieser Patientenagenda anzunehmen.

Der Kranke erwartet von der Medizin die Berücksichtigung seiner seelischen und sozialen Bedingungen und Bedürfnisse (Schipperges 1990). Patienten erwarten von der hausärztlichen Konsultation nicht nur Heilung oder Befreiung von Beschwerden, sondern auch Kommunikation, Information und Einfühlungsvermögen (Kochen 1999). Vom Arzt der Zukunft erwartet der Patient nicht nur den raschen und oft lebensrettenden Eingriff, sondern mehr und mehr auch das Eingehen auf die sozialen Krisenfelder, eine Zuwendung und Begleitung bei chronischen Leiden, eine Beratung und Führung in Fragen eines gesundheitsbewussten Lebensstils (Schipperges 1990). Die vorrangige Erwartung der Patienten gilt der verständlichen Erläuterung der Diagnose und Therapie durch den Hausarzt und einer freundlichen und verständnisvollen Umgangsart miteinander (Southgate, Bass 1983).

Skelton et al. (1996) untersuchten die Sicht der Patienten über KS und deren Zusammenhänge in der Allgemeinarztpraxis. Es wurden Patienteninterviews durchgeführt und danach analysiert, bezogen auf folgende sechs Themen: Lebensqualität, Prognose, sekundäre Prävention, Bereitschaft, einen Arzt zu 
konsultieren, Zufriedenheit mit der Erklärung der Schmerzen, Konsultation eines Ersatztherapeuten (z.B. Osteopathen oder Chiropraktiker). Dabei wurde festgestellt, dass die Meinungen der Patienten mit KS heterogener Art sind. Jedes Individuum betrachtet das Problem KS, welches als "gleiche Bedingung" letztendlich allen Patienten zugrunde liegt, auf ganz unterschiedliche Art und Weise. Deshalb sollte der gesamte Gesundheitszustand des Patienten in Betracht gezogen werden, um eine optimale Behandlung der Schmerzen zu garantieren. Die Studie hebt außerdem hervor, dass Patienten keine magische Heilung ihrer KS erwarten, sondern sich von ihrem HA Zuhören und ein verständliches Behandlungsmanagement wünschen. Im gleichen zeitlichen und örtlichen Rahmen wurden mit den $\mathrm{HÄ}$ semistrukturierte Interviews bezüglich der Wahrnehmung von Kreuzschmerzpatienten durchgeführt und separat ausgewertet (Skelton et al. 1995). Die Studienanalyse zeigt sechs wichtige Prinzipien (z.B. psychologische Konstitution des Patienten, klinischer Status, Beruf, soziale Gruppierung etc.), mit Hilfe derer die HÄ verschiedene Patienten unterscheiden und passende Behandlungen einleiten. Die Ergebnisse zeigen, dass diese Einteilung, welche die HÄ nutzen, die Vielfalt an Perspektiven, Bedürfnissen und Erfahrungen der Patienten mit KS grob vereinfacht und letztlich $\mathrm{zu}$ Unstimmigkeiten im Verstehen und in den Erwartungen zwischen Patient und HA führen kann.

In einer australischen Studie über Patientenerwartungen (Cockburn et al. 1997) wird berichtet, dass Patienten, trotz eigener Erwartungen hinsichtlich der Medikation, die Meinung ihres HA und dessen Verordnung akzeptierten. 


\section{Material und Methoden}

\subsection{Studiendesign}

Bei dieser Studie handelt es sich um eine prospektive Kohortenstudie mit einem Follow-up-Zeitraum von 3 Monaten. Patienten, die am Tag der Hausarztkonsultation unter KS leiden, werden eingeladen, sich an der Studie zu beteiligen und einen FB auszufüllen. Diejenigen, die zusätzlich mit einem bzw. zwei Telefoninterviews einverstanden sind, werden zwei Wochen und drei Monate nach der Arztkonsultation kontaktiert. An das 2. Telefoninterview nach 3 Monaten schließt sich eine zusätzliche Befragung der Patienten mittels eines Schmerztagebuchs an. Diese Befragung gilt als Pilotierung im Rahmen eines vom Bundesministerium für Bildung und Forschung geförderten Rückenschmerzprojekts.

Während eines Untersuchungszeitraums (eine Arbeitswoche von Montag bis Freitag) wurden Patienten konsekutiv rekrutiert und gebeten, vor der Arztkonsultation einen FB auszufüllen. Die Patienten, die sich zu einer weiteren Erhebung bereit erklärten, wurden 2 Wochen und nochmals 3 Monate nach der Hausarztkonsultation in einem Telefoninterview zu ihren Beschwerden befragt.

Die Ärzte, die ihr Einverständnis zur Studie erklärten, wurden gebeten, eine Strichliste mit allen an KS leidenden Patienten zu führen, die sie in dieser Woche konsultierten.

In der folgenden Abbildung sind die Themen der jeweiligen Befragung in einer Übersicht aufgelistet; ausführliche Beschreibungen finden sich in den Unterpunkten $3.2,3.3$, und 3.4 . 


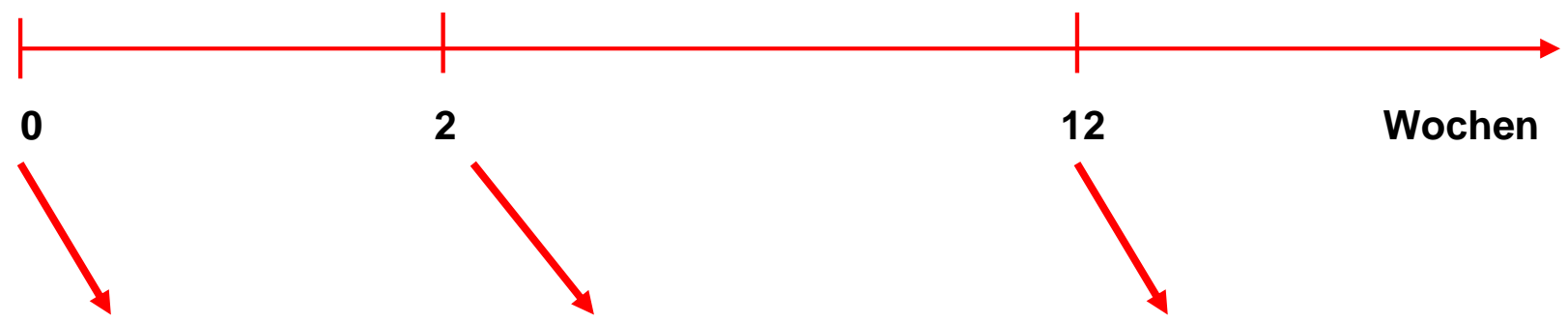

Epidemiologie

$>$ Inzidenz von KS

\section{Schmerzerleben}

$>$ Schmerzintensität

Patienteneinstellung

$>$ Selbstbewältigung

$>$ Beste Behandlung aus Patientensicht

$>$ Weitere Untersuchungen

\section{Erwartungen an die} hausärztliche Konsultation

$>$ Das Wichtigste für den Patienten beim Arztbesuch

$>$ Möglichkeit für offene Fragen an den Arzt

\section{Versorgung beim} Hausarztbesuch

Verordnungen, weitere Behandlungen

> Weitere Diagnostik

> Ärztliche Empfehlungen

$>\mathrm{AU}$, Rentenantrag

$>$ Erfüllung der Erwartung an den Hausarztbesuch

\section{Schmerzerleben}

$>$ Schmerzintensität

\section{Krankheitsverlauf}

$>$ Einschränkung durch die Schmerzen

$>$ Prognose

$>$ Wohlbefinden

\section{Patienteneinstellung}

$>$ Selbsthilfe

$>$ Beweglichkeit

$>$ Körperliche Aktivität

\section{Epidemiologie}

$>$ Punktprävalenz nach Follow-up

\section{Versorgungs/eistungen im} Follow-up

$>$ Arztbesuche

$>$ Verordnungen, weitere Behandlungen

\section{Schmerzerleben}

Schmerzintensität

\section{Krankheitsverlauf}

$>$ Einschränkung durch die Schmerzen

$>$ Wohlbefinden

\section{Patienteneinstellung}

$>$ Körperliche Aktivität

$>$ Selbsthilfe

Abbildung 2: Studiendesign

\subsection{Patientenfragebogen}

Der Patientenfragebogen (s. Anhang) enthielt auf 2 Seiten neben Angaben zu Alter und Geschlecht Fragen zu den aktuellen Beschwerden, zur Epidemiologie sowie zu den Erwartungen des Patienten an die hausärztliche Konsultation: 
- Inzidenz von KS

- Schmerzintensität

- Selbstbewältigungsstrategien

- Frage nach bester Behandlung aus Patientensicht

- Wunsch nach weiterer Diagnostik

- Ziel des Hausarztbesuches

- Offene Fragen an den Arzt in Bezug auf die Schmerzen.

Neben Alternativfragen wie "Ja oder Nein" hatten die Patienten die Möglichkeit, Mehrfachantworten zu geben oder auch unter "Sonstigem“ ihre eigenen Kommentare hinzuzufügen. Als Abschlussblatt diente die nötige Einverständniserklärung eines jeden Patienten, seine Daten auswerten zu lassen. Weiterhin gab es die Möglichkeit, für ein bzw. zwei Telefoninterviews Name und Telefonnummer zu hinterlassen.

\subsection{Fragebogen zum 1. Telefoninterview}

Das Telefoninterview (s. Anhang) wurde mit den Patienten, die ihren Namen und ihre Telefonnummer auf dem FB in der Praxis hinterlassen hatten, innerhalb von 2 Wochen nach der Arztkonsultation durchgeführt. Das Gespräch schloss Fragen zur hausärztlichen Behandlung und zur Erfüllung der Erwartung an den Hausarztbesuch ein:

- Verschreibung von Medikamenten, Gabe von Injektionen

- Verordnung anderer Behandlungen (Krankengymnastik, Akupunktur) oder weiterer Diagnostik (z.B. Überweisung zum Facharzt oder zum Röntgen)

- Ärztliche Empfehlungen für den Patienten

- Ausstellung einer Arbeitsunfähigkeitsbescheinigung oder Antrag auf einen Rentenantrag

- Erwartungen des Patienten an den Hausarztbesuch und Frage nach Erfüllung dergleichen. 
Der 2. Teil des Telefongesprächs enthielt Fragen aus einem etwas erweiterten Schmerztagebuch. Die Originalvorlage dieses Schmerztagebuchs wurde freundlicherweise von Herrn Prof. Basler, Abteilung Medizinische Psychologie der Philipps Universität Marburg zur Verfügung gestellt. Die Fragen bezogen sich hauptsächlich auf die vergangenen 24 Stunden und beinhalteten Themen zur Schmerzausprägung sowie zur subjektiven Krankheitswahrnehmung und Krankheitsentwicklung. Auf einer Numerischen Analogskala (0 bis 10), die jedes Mal für die entsprechende Frage neu erläutert wurde, konnten die Patienten ihre individuelle Antwort geben. Erneut gab es Alternativfragen wie „Ja oder Nein“, Freitext oder Angaben unter „Sonstiges“:

- Schmerzintensität und körperliche Einschränkung durch die Schmerzen

- Einflussnahme auf die Schmerzen außer durch Medikamente

- Verbesserung der Beweglichkeit

- Aktivität in den letzten 24 Stunden (in Minuten)

- Wohlbefinden in den letzten 24 Stunden

- Frage nach der möglichen Schmerzursache

- Hoffnung des Patienten auf Besserung

- Auswirkung der Stimmung des Patienten auf die Schmerzen.

Am Ende des Gesprächs wurde der Patient erneut gefragt, ob er mit einem weiteren Telefoninterview in ca. 3 Monaten einverstanden sei.

\subsection{Fragebogen zum 2. Telefoninterview}

Nach 3 Monaten wurden die Patienten erneut kontaktiert. In diesem Telefonat dominierten Fragen über die Versorgungsleistungen in den letzen 12 Wochen und Fragen nach der Umsetzung der Empfehlungen:

- Aktuelle Beschwerden, Häufigkeit und Intensität von KS in den vergangenen 3 Monaten 
- Konsultation eines Arztes wegen der bestehenden Schmerzen

- Fragen zu diesem Arztbesuch (Medikamente, Spritzen, andere Behandlungen)

- Rückblick auf die vergangenen 3 Monate hinsichtlich Aktivität und Selbstbewältigungsstrategien.

Um den Krankheitsverlauf zu beobachten, wurden im 2. Teil des Gesprächs erneut Fragen bezogen auf die letzten 24 Stunden gestellt, die teilweise wieder mit der o.g. Zehner-Skala beantwortet werden konnten:

- Schmerzintensität und körperliche Beeinträchtigung durch die Schmerzen

- Sportliche Aktivität (Minutenangabe)

- Frage nach dem Wohlbefinden am Tag des Interviews.

Am Ende dieses Interviews wurden die Patienten gefragt, ob sie sich bereit erklären, ein Schmerztagebuch, welches innen ein paar Tage später zugeschickt werden würde, auszufüllen. Haben die Patienten zugesagt, wurden Name und Adresse notiert, um Ihnen die Bücher zuzuschicken.

\subsection{Das Schmerztagebuch}

Der Patient wurde gebeten, an sieben aufeinanderfolgenden Tagen sechs jeweils gleiche Fragen zu beantworten (s. Anhang), die sich erneut auf die letzten 24 Stunden beziehen. Die teilweise Wiederholung der bereits telefonisch abgefragten Items fungierte hier zur Pilotierung der schriftlichen Befragung und der Compliance im Umgang mit den Tagebüchern. Der Patient hatte abermals die Möglichkeit auf einer Zehner-Skala die für inn passende Antwort auszuwählen:

- Schmerzintensität

- Selbstmedikation und Hausarztkonsultation

- Selbstbewältigungsstrategien, außer Medikamente

- Einschränkungen durch die Schmerzen in Tätigkeiten und Bedürfnissen 
- Sportliche Aktivität (Angabe in Minuten und offener Text)

- Frage nach dem Wohlbefinden am Tag des Interviews.

Jedem Tagebuch war ein frankierter, an die Abteilung Allgemeinmedizin adressierter, Rückumschlag beigelegt, in welchem das Tagebuch zurückgesandt werden konnte. Die Schmerztagebücher waren symbolisch gekennzeichnet, um die spätere Zuordnung zu den Angaben in den Telefoninterviews zu erleichtern. Selbstverständlich waren Rückschlüsse auf die Identität der Patienten nicht möglich.

\subsection{Pilotstudie}

Der FB und das erste Telefoninterview wurden in einer Allgemeinarztpraxis im Raum Vechta, Landkreis Südoldenburg, im Februar 2001 pilotiert. Innerhalb einer Woche haben sich 11 Patienten zur Teilnahme bereit erklärt und davon gaben 5 Patienten $(45,5 \%)$ ihr Einverständnis zum anschließenden Telefoninterview.

Anhand der Auswertung wurde der Patientenfragebogen verbessert und überarbeitet, um Unklarheiten und unpräzise Antworten zu umgehen.

Außerdem wurde kurz mit dem Arzt und dessen Helferinnen Rücksprache gehalten, um Rückmeldung zum Studienablauf in der Praxis zu erhalten und dies in der Hauptstudie zu berücksichtigen. Es zeigte sich, dass Plakate im Wartezimmer und Empfangsbereich sowie die Auslage von Handzetteln allein nicht ausreichten, die Patienten genügend auf die Studie aufmerksam zu machen. Folglich resultierte die Überlegung, dass es für die Patientenrekrutierung besonders hilfreich ist, wenn die Arzthelferinnen zusätzlich auf die Studie bzw. auf die Plakate und Handzettel verwiesen.

\subsection{Auswahl der Arztpraxen}

Im Kreis Vechta und Cloppenburg, Landkreis Südoldenburg, wurden Anfang April 2001 für die Studie 35 Allgemeinarztpraxen mit Hilfe des Ärzteverzeichnisses ausgewählt und angeschrieben. Im Brief wurden das Ziel der Studie und der geplante Ablauf geschildert. Eine Rückantwort in Form eines Fax, sowie 
Kontaktnummern der Abteilung Allgemeinmedizin Göttingen und der Doktorandin für eventuelle Rückfragen wurden beigelegt. Wenn bis zu 10 Tagen später keine Antwort erfolgte, wurden die Ärzte telefonisch persönlich kontaktiert und um Teilnahme geworben. Gegebenenfalls erfolgte dann die Terminabsprache für den Studienbeginn.

\subsection{Auswahl der Patienten und Ablauf der Patientenbefragung}

Die niedergelassenen Ärzte, die sich zur Teilnahme bereit erklärten, wurden zum vereinbarten Termin von der Doktorandin aufgesucht. Das Material (Plakate, Handzettel und Fragebögen) wurde in jeder Praxis verteilt und erläutert. Gemeinsam mit den Arzthelferinnen wurde der Studienablauf besprochen. Sie sollten für die Patienten Ansprechpartner bei Fragen oder Unklarheiten sein. Im Wartezimmer und Empfangsbereich wurden je ein Plakat als erster Blickfang angebracht. Am 1. Tag der Studie (Montags) lagen am Tresen und im Wartezimmer viele Handzettel (s. Anhang) sichtbar aus, um die Patienten auf die Studie aufmerksam zu machen. Diese Zettel enthielten Informationen zur Studie und den Hinweis, bei Interesse einen FB an der Anmeldung abholen zu können. Die Arzthelferinnen wurden gebeten, alle Patienten mit Rückenschmerzen anzusprechen und um Teilnahme an der Studie zu bitten.

Die Patienten, die sich zur Mitarbeit bereit erklärten, bekamen diesen FB, Unterlage und Stift von den Arzthelferinnen ausgeteilt. Wichtig war das Ausfüllen des FB vor der eigentlichen Arztkonsultation. Das Lesen und Ausfüllen dauerte im Durchschnitt zwischen 5-10 Minuten. Anschließend wurde der Bogen von den Helferinnen entgegengenommen und in einen bereitgestellten Ordner bis zum Ende der Woche aufbewahrt.

Die Arzthelferinnen notierten auf einem vorgefertigten Zettel (s. Anhang) die Gesamtzahl der Patienten, die am jeweiligen Tag die Praxis aufsuchten.

Auch dem Arzt wurde eine Strichliste zur Verfügung gestellt (s. Anhang). Er wurde gebeten, die Anzahl der Patienten zu notieren, die an diesem Tag tatsächlich wegen KS die Sprechstunde aufsuchten - unabhängig davon, ob sie vorher den FB 
ausgefüllt hatten oder nicht. Den FB nahm der Patient nicht mit in das Sprechzimmer, sondern gab ihn vor der Arztkonsultation an der Anmeldung ab.

\subsection{Auswertung}

Die Auswertung erfolgt hinsichtlich der Fragen zur Schmerzcharakteristik sowie der (versorgungs-) epidemiologischen Daten deskriptiv (Häufigkeitsauszählungen) mit dem Statistikprogramm SAS 8.2 (SAS Cary, NC, USA).

Zur Darstellung der Veränderungen von Schmerzintensität, Einschränkungen durch und Einflussnahme auf die Schmerzen und Wohlbefinden im zeitlichen Verlauf (nach 2 und 14 Wochen) wurden chi²-Tests mit einem Signifikanzniveau von 5\% durchgeführt. 


\section{Ergebnisse}

\subsection{Teilnahmebereitschaft an der Studie}

\subsection{1 Ärzte}

Insgesamt wurden 35 Fachärzte für Allgemeinmedizin (27 Arztpraxen, davon 5 Gemeinschaftspraxen) im Kreis Vechta und Cloppenburg, Bezirk Südoldenburg angeschrieben. Auf das Anschreiben, welches im April 2001 an alle Ärzte verschickt wurde, meldeten sich spontan 3 Ärzte in 2 Praxen per Rückfax. Um die Teilnehmerquote zu verbessern, wurden die Ärzte Mitte April 2001 zusätzlich telefonisch kontaktiert. Hierauf klärten sich 14 weitere Ärzte in 13 Praxen zur Studienteilnahme bereit. Insgesamt gaben von 35 Ärzten in 27 Praxen 17 Ärzte in 15 Praxen (49\%) ihr Einverständnis zur Teilnahme an der Studie. 8 Allgemeinmediziner lehnten eine Teilnahme am Telefon, 4 weitere per Faxantwort ab. Vorwiegend wurden Zeitmangel, Unzumutbarkeit für die Patienten und Sorge um ein „Durcheinander“ im Praxisablauf als Gründe genannt.

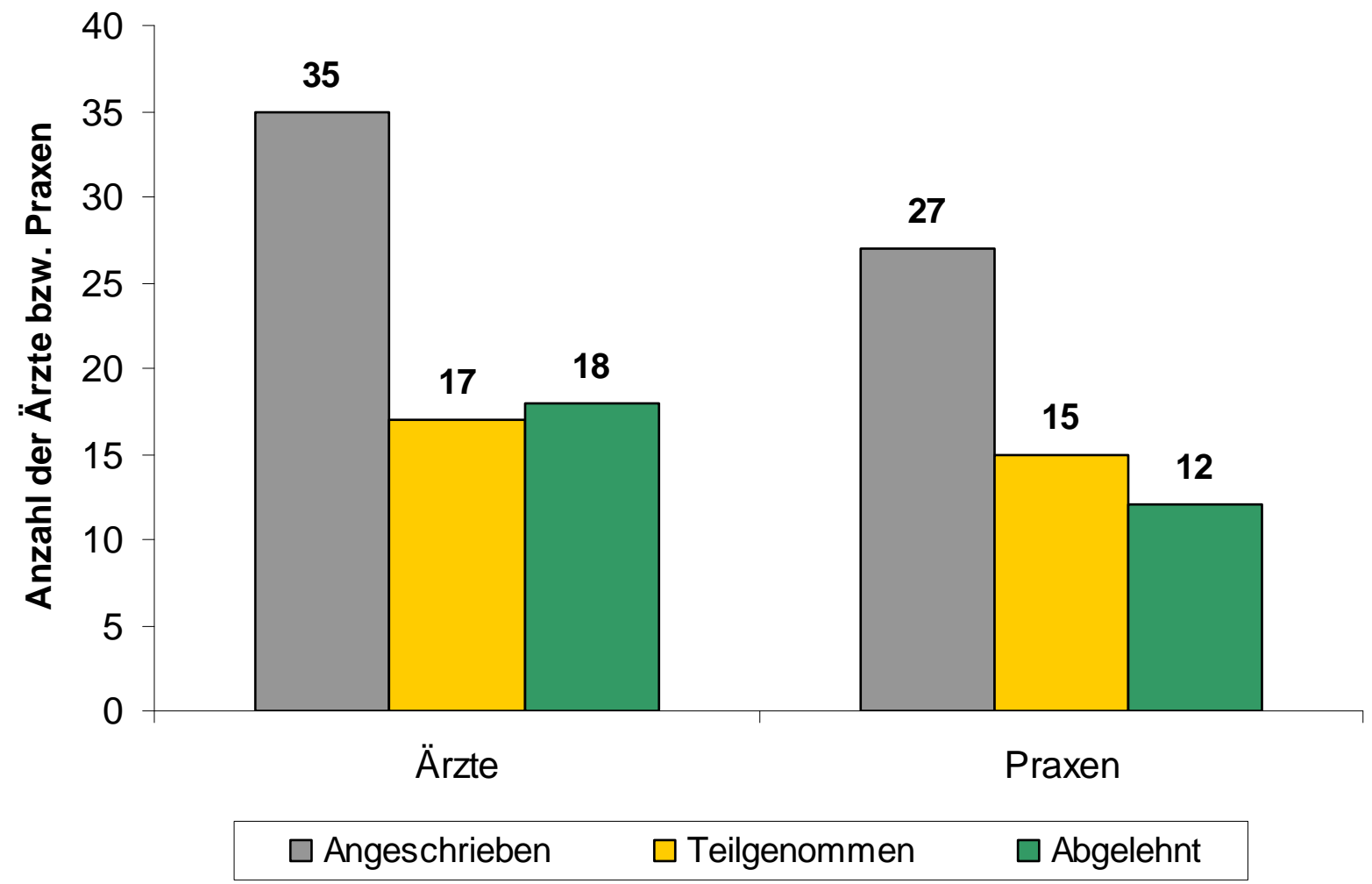

Abbildung 3: Teilnahmebereitschaft der Arztpraxen 


\subsubsection{Patientenbeteiligung}

Die Patientenbeteiligung in den einzelnen Praxen fiel sehr unterschiedlich aus. Insgesamt wurden in den 15 Arztpraxen 127 Fragebögen von Patienten mit KS ausgefüllt, das sind durchschnittlich 8,46 pro Praxis (0-17 FB). 6 Patienten haben keine Altersangabe vermerkt. Das Durchschnittsalter lag bei 47,94 Jahren (17-89, Median: 53 Jahre). Es haben 69 Frauen (54,3\%) und 58 Männer (45,7\%) den FB vor der Arztkonsultation ausgefüllt. Es nahmen fast zwei Drittel der Patienten, die in der Untersuchungswoche ihren HA mit KS konsultierten (s. Tabelle 1) an der Studie teil; die Responsrate betrug $64,5 \%$.

Am Ende des FB gab es für die Patienten die Möglichkeit, ihr Einverständnis zu einem Telefoninterview 2 Wochen nach der Arztkonsultation zu geben. Hierfür notierten sie ihren Namen und ihre Telefonnummer in der Einverständniserklärung. Für das erste Telefoninterview nach 2 Wochen gaben 50 Patienten (39,4\%) eine schriftliche Zusage. Bis auf einen konnten alle 49 (38,6\%) Patienten telefonisch erreicht und befragt werden. Im zweiten Telefonat nach 3 Monaten berichteten 40 von 49 Patienten (81,6\%) nochmals über ihre Beschwerden. Neun Patienten waren zu diesem Zeitpunkt nicht erreichbar. Das Schmerztagebuch füllten 26 Patienten von 40 (65\%) zwei Wochen später aus (Abbildung 4).

\section{$\underline{\text { Ausgefüllte Fragebogen } \quad \text { 2. Telefoninterview }}$}

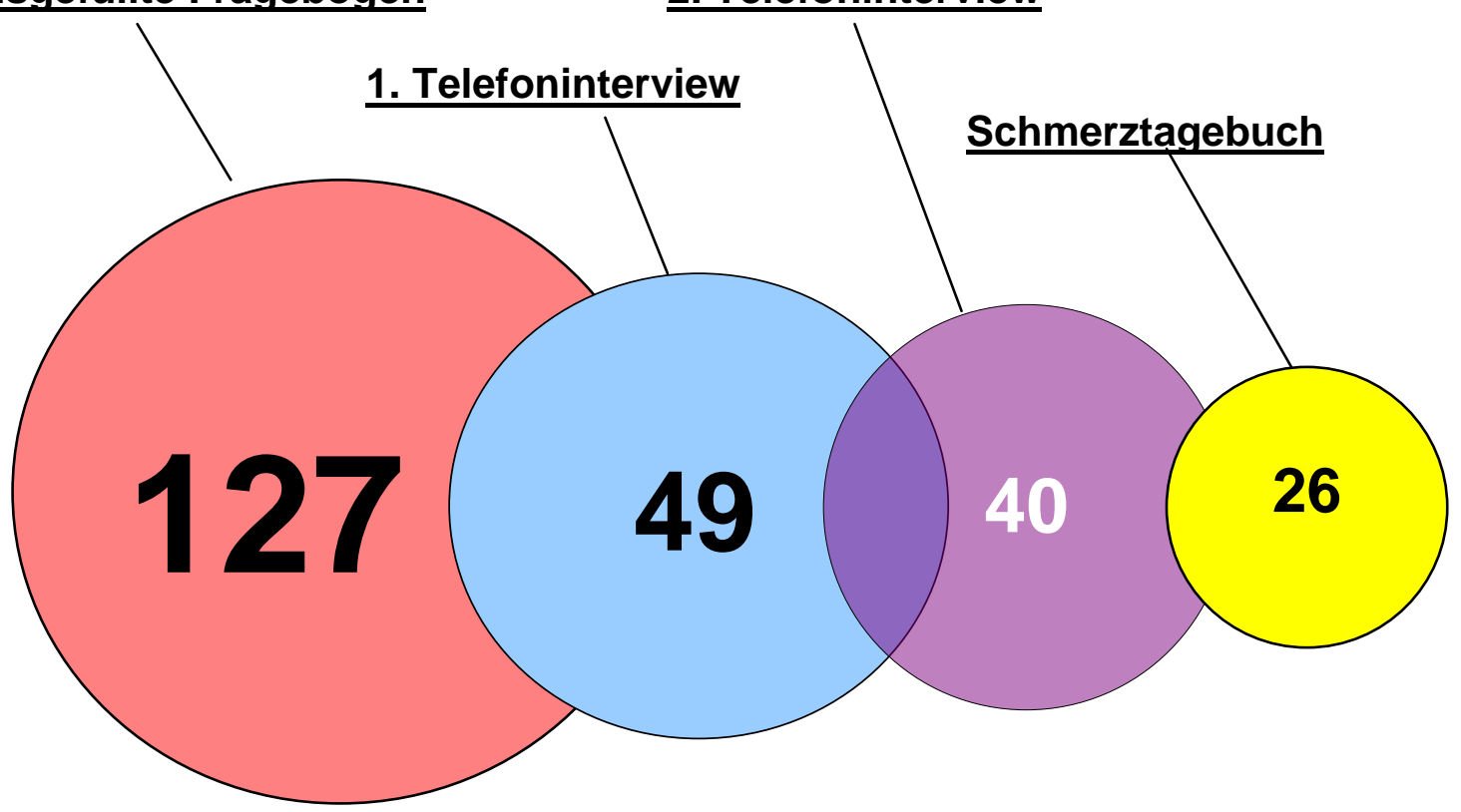

Abbildung 4: Teilnahmebereitschaft der Patienten 
Die Abbildung 5 demonstriert die Zuordnung der Kreuzschmerzpatienten in Altersgruppen. Am häufigsten waren Frauen im Alter von 25 bis 64 Jahren wegen KS bei ihrem HA, wobei die Altersgruppe zwischen 45 und 54 Jahren am stärksten vertreten war $(n=13)$. Bei den männlichen Patienten zeichneten sich 2 Altersgipfel ab. 25 bis 44-jährige und Männer im Alter von 55 bis 74 Jahren konsultierten im Untersuchungszeitraum ihren HA aufgrund von KS am häufigsten.

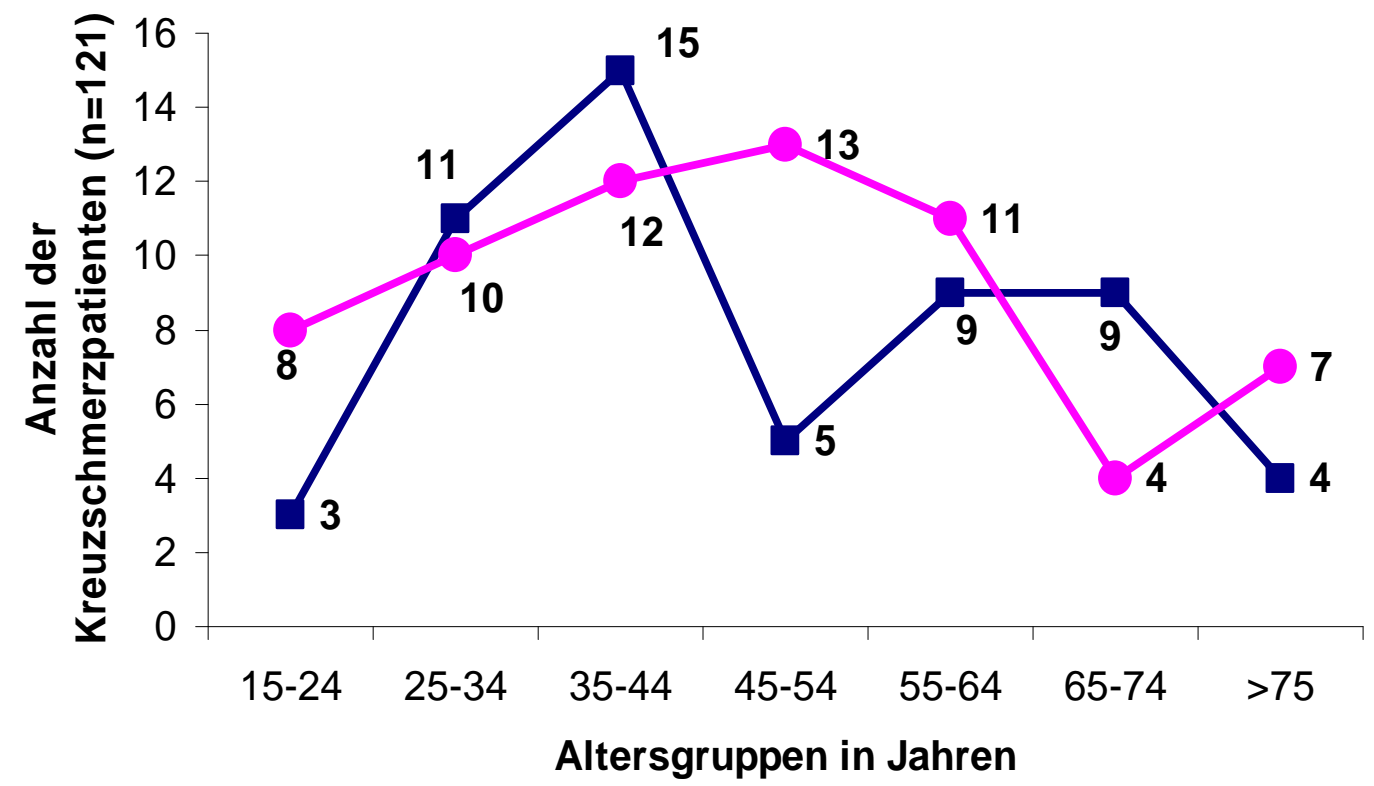

- männlich weiblich

Abbildung 5: Anzahl der Kreuzschmerzpatienten in Arztpraxen nach Zuordnung in Altersgruppen ( $\mathrm{n}=$ 121)

Zum Zeitpunkt der Befragung 2 Wochen nach dem Hausarztbesuch waren 61,2\% der 49 befragten Patienten berufstätig, 30,6\% Rentner und 4,1\% Hausfrauen (weitere $4,1 \%$ hatten keine Berufsangabe gemacht).

\subsection{Epidemiologie von Kreuzschmerzen in der hausärztlichen Praxis}

\subsubsection{Praxisprävalenz}

Die Ärzte wurden gebeten, auf einer vorbereiteten Strichliste (s. Anhang) täglich alle Patienten, welche die Sprechstunde wegen KS an diesem Tag aufgesucht haben, zu vermerken. 10 der 17 Ärzte (59\%) füllten diese Liste für die 5 Tage aus, an denen die 
Studie lief (Montagmorgen bis Freitagabend). Weiterhin wurden die Arzthelferinnen gebeten, in dieser einen Arbeitswoche eine Liste (s. Anhang) am Tresen zu führen, welche alle Patienten des Tages (unabhängig vom Konsultationsgrund), die die Praxis aufgesucht haben, erfasste. Hier kooperierten erneut die 8 Praxen, deren Ärzte auch schon die o.g. Strichliste ausgefüllt haben. Die sieben übrigen Ärzte lehnten das Ausfüllen beider Listen ab, wobei hier als Gründe unter anderem „Zu sehr praxisintern“, „Unbehagen, dass Zahlen nach draußen gelangen könnten“, genannt worden sind. Die nachfolgenden Zahlen dieses Unterpunktes beziehen sich auf diese 8 Praxen. In dem Zeitraum einer Woche haben insgesamt 2909 Patienten (im Mittel 363 Patienten/ Woche/ Praxis) ihre Hausarztpraxis aufgesucht, unabhängig davon, ob sie den Arzt konsultiert oder ein Rezept abgeholt haben. Davon waren 197 Patienten auf den Strichlisten der Ärzte als „Patienten mit Kreuzschmerzen, die heute Ihre Sprechstunde aufsuchen“ notiert. Die mittlere 1-Wochen-Prävalenz von Kreuzschmerzpatienten beträgt 7,9\% (Tabelle 1).

Tabelle 1: Praxenübersicht mit Angabe der mittleren 1-Wochen-Praxisprävalenz

\begin{tabular}{|c|c|c|c|c|c|c|c|c|c|}
\hline & Praxen & 1 & 2 & 3 & 4 & 5 & 6 & 7 & 8 \\
\hline \multirow[t]{2}{*}{ Montag } & $A$ & 96 & 53 & 115 & 63 & 61 & 105 & 59 & 123 \\
\hline & $\mathrm{B}$ & 10 & 11 & 7 & 10 & 7 & 5 & 2 & 4 \\
\hline \multirow[t]{2}{*}{ Dienstag } & $A$ & 62 & 61 & 92 & 45 & 51 & 96 & 82 & 134 \\
\hline & $\mathrm{B}$ & 3 & 8 & 5 & 6 & 6 & 6 & 2 & 0 \\
\hline \multirow[t]{2}{*}{ Mittwoch } & $A$ & 44 & 41 & 69 & 46 & 46 & 67 & 41 & 110 \\
\hline & $\mathrm{B}$ & 8 & 2 & 3 & 8 & 8 & 3 & 2 & 1 \\
\hline \multirow[t]{2}{*}{ Donnerstag } & $A$ & 72 & 66 & 80 & 54 & 60 & 77 & 78 & 111 \\
\hline & $\mathrm{B}$ & 4 & 7 & 8 & 4 & 7 & 2 & 10 & 3 \\
\hline \multirow[t]{2}{*}{ Freitag } & $A$ & 81 & 50 & 94 & 32 & 49 & 83 & 43 & 117 \\
\hline & B & 5 & 4 & 1 & 2 & 8 & 2 & 0 & 2 \\
\hline \multirow[t]{2}{*}{ Gesamt } & $\mathrm{A}$ & 355 & 271 & 450 & 240 & 267 & 428 & 303 & 595 \\
\hline & $\begin{array}{c}\text { B } \\
\text { (insg. }=197)\end{array}$ & 30 & 32 & 24 & 30 & 36 & 18 & 17 & 10 \\
\hline \multicolumn{2}{|c|}{$\begin{array}{l}\text { Praxisprävalenz } \\
\text { (in \%) }\end{array}$} & 8,5 & 11.8 & 5,3 & 12,5 & 13,5 & 4,2 & 5,6 & 1,7 \\
\hline
\end{tabular}

\footnotetext{
$A=$ Gesamtzahl der Patienten in der Praxis

$B=$ Anzahl der Kreuzschmerzpatienten in der Praxis
} 


\subsubsection{Punktprävalenz nach dem Follow-up}

3 Monate nach dem Arztbesuch hatten noch 60\% der 40 befragten Patienten KS.

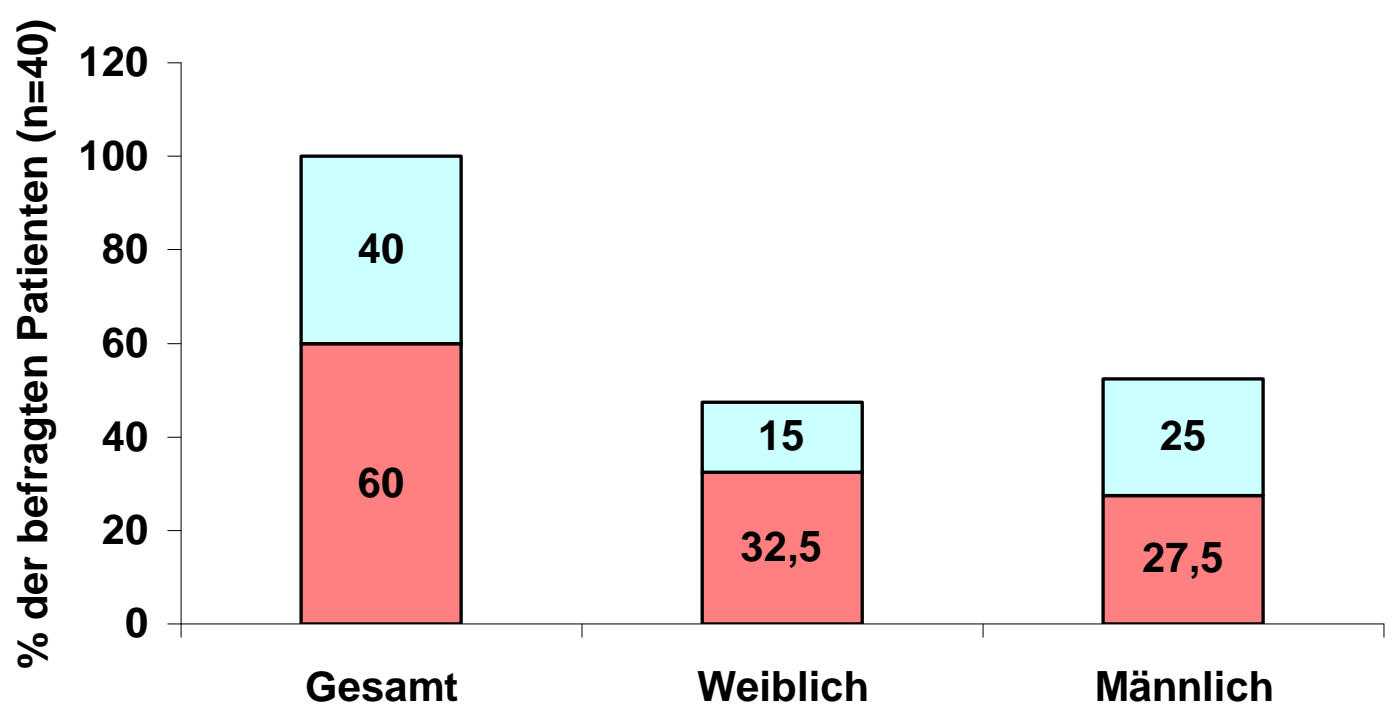

Abbildung 6: Punktprävalenz von Kreuzschmerzen nach 3 Monaten $(n=40)$

Die durchschnittliche Schmerzstärke auf der Skala von 0 bis $10(0=$ gar keine Schmerzen, 10= maximal denkbare Schmerzen) lag, bezogen auf die letzten 24 Stunden, bei 4,7 .

\subsubsection{Inzidenz}

Die Anzahl der Neuerkrankungen, bezogen auf die Gesamtzahl der Patienten mit KS im Untersuchungszeitraum der Studie, betrug 7. Dies entspricht einer Inzidenzrate von $5,5 \%$.

\subsubsection{Rezidivrate}

Bei 19,2\% der befragten Patienten ( $n=120$ ) lag die letzte Episode mit KS mehr als 6 Monate zurück. Das bedeutet nach einem symptomfreien Intervall von mindestens einem halben Jahr traten bei diesen Patienten erneut Schmerzen in der unteren Lendengegend auf, weshalb der HA konsultiert wurde.

Nach 12 Wochen wurden die Patienten erneut nach aufgetretenen KS innerhalb der letzten 3 Monate befragt. 8\% hatten keine Beschwerden in diesem Zeitraum („nie“). 
Bei 37\% (,selten“) traten vereinzelt an nicht aufeinanderfolgenden Tagen Schmerzen auf. Ein Viertel klagte über häufige Schmerzepisoden mindestens einmal in der Woche („wöchentlich“). 30\% der Befragten litten täglich unter KS (Abbildung 7).

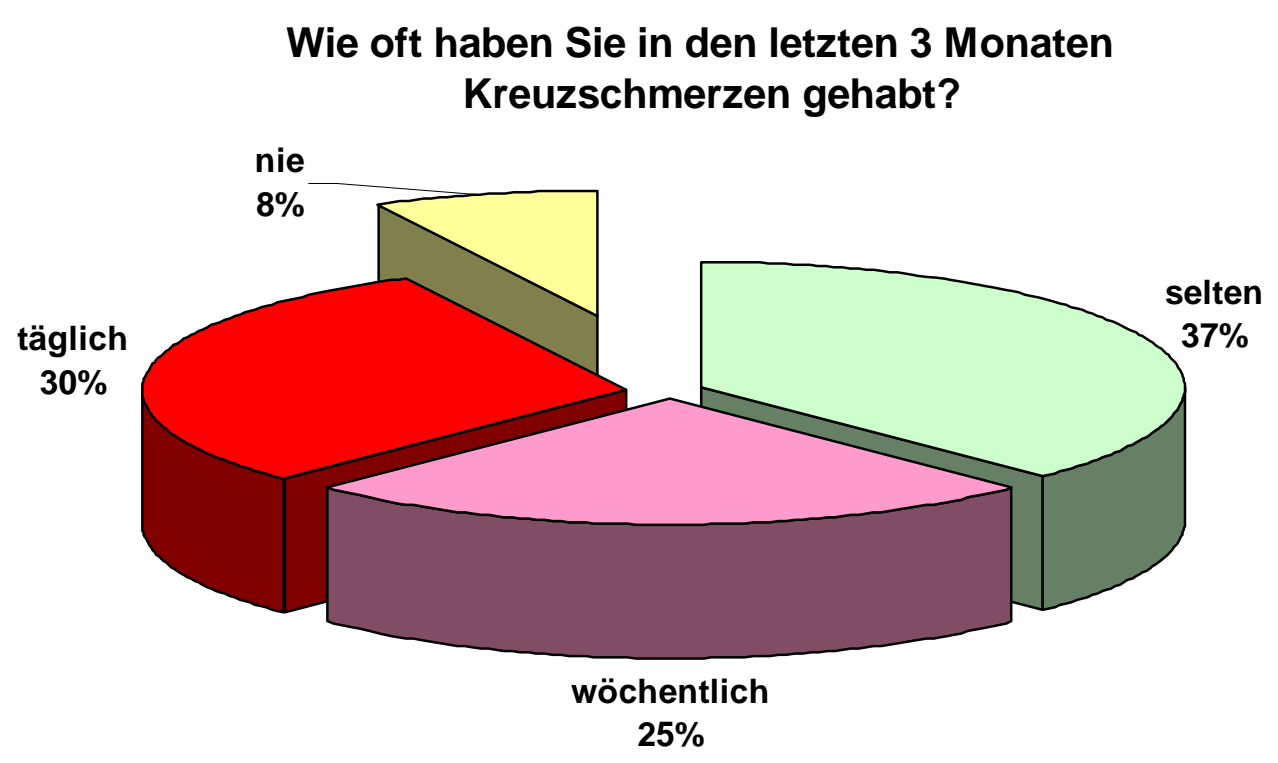

Abbildung 7: Häufigkeit auftretender Kreuzschmerzen während des Follow-up ( $n=40$ )

\subsection{Inanspruchnahme von Versorgungsleistungen}

\subsubsection{Hausärztliche Behandlung $(n=49)$}

In diesem Kapitel wird über die hausärztliche Behandlung zum Rekrutierungszeitpunkt berichtet. Die folgenden Angaben beziehen sich auf die 49 von 127 Patienten, die in dem ersten Telefoninterview 2 Wochen nach der Arztkonsultation bereit waren, Fragen zu beantworten. 


\subsubsection{Verschreibung von Medikamenten}

Fast $42 \%$ der Patienten ( $n=48$ ) gaben an, Medikamente von ihrem HA verschrieben bekommen zu haben.

60\% aller Verordnungen machten NSAR aus. Die stärksten Vertreter dieser Gruppe waren Ibuprofen, Diclofenac und Naproxen. Bei den opioiden Analgetika verordneten die HÄ hoch potente (8\%, Fentanyl, Morphium) und niedrig potente (4\%, Codein, Tramadol). Ebenso häufig (12\%) wurden andere zentral wirksame Mittel gegen Störungen des Muskel- und Skelettsystems wie Tolperison und Tizanidin verschrieben. Andere Analgetika wie Flupirtin (8\%), Antidepressiva vom Amitriptylintyp (4\%) und Vitamin D3 - Präparate (Colecalciferol) wurden seltener verordnet wie aus Abbildung 8 zu erkennen ist.

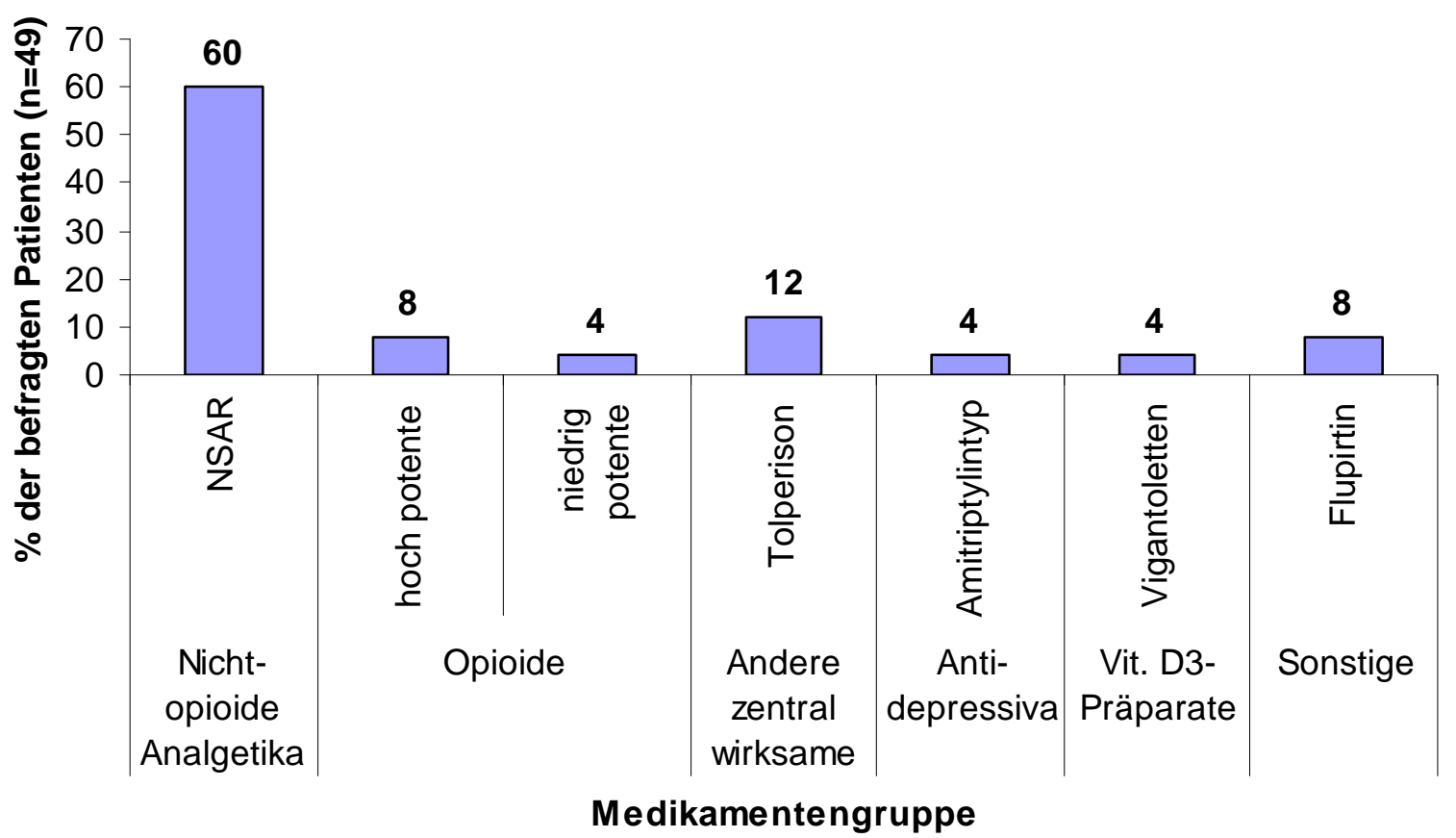

Abbildung 8: Medikamente mit Namen der Wirkgruppe und Zahl der Verordnungen in \% 


\subsubsection{Verabreichung von Injektionen}

Über 40\% der befragten Patienten ( $n=49)$ erhielten am Tag der Hausarztkonsultation eine Spritze, wobei mehr als zwei Drittel davon (28,6\%) intramuskulär injiziert wurden. 5 Patienten (10,2\%) wurde eine Injektion in den Rücken (paravertebral) verabreicht und zwei $(4,1 \%)$ wurden gequaddelt (Tabelle 2).

Tabelle 2: Gabe von Injektionen bei der Hausarztkonsultation ( $n=49)$

\begin{tabular}{|c|c|}
\hline Art der Injektion & $\mathbf{n}(\%)$ \\
\hline intramuskuläre Spritze & $14(28,6 \%)$ \\
\hline paravertebrale Injektion & $5(10,2 \%)$ \\
\hline Quaddeln setzen & $2(4,1 \%)$ \\
\hline
\end{tabular}

Zu erwähnen sei in diesem Zusammenhang der direkte Vergleich zwischen den Punkten 4.3.1.1 und 4.3.1.2, aus dem hervorgeht, wie viele Patienten welche Art von Behandlung bezogen haben. Von 49 Patienten bekamen 12 (24,5\%) nur ein Medikament, 13 (26,5\%) nur eine Spritze gegen die Schmerzen, 8 (16,3\%) ein Medikament und eine Injektion und 16 Patienten $(32,7 \%)$ keines von beiden.

\subsubsection{Durchführung weiterer Behandlungen}

Über der Hälfte der Patienten (51\%, n=49) wurde am Tag der Arztkonsultation eine weitere Behandlung empfohlen (z.B. Akupunktur) bzw. verschrieben (z.B. Krankengymnastik). Am häufigsten wurde Krankengymnastik und Kurzwelle eingesetzt (s. Tabelle 3). 
Tabelle 3: Durchführung weiterer Behandlungen am Tag der Hausarztkonsultation $(n=49)$

\begin{tabular}{|c|c|}
\hline Art der Behandlung & $\%$ \\
\hline Krankengymnastik & 14,3 \\
\hline Kurzwelle & 10,2 \\
\hline Massage & 8,2 \\
\hline Reizstrom & 8,2 \\
\hline Akupunktur & 4,1 \\
\hline Einrenken & 4,1 \\
\hline Manuelle Therapie & 2 \\
\hline Keine Behandlung & 49 \\
\hline
\end{tabular}

\subsubsection{4 Überweisung an einen Facharzt}

6 der 49 Patienten erhielten beim Besuch ihres HA eine Überweisung zu einem Facharzt. Fünf dieser Patienten stellten sich bei einem Orthopäden und einer bei einem Neurologen vor.

\subsubsection{Bildgebende Verfahren}

Unabhängig von den Überweisungen wurden bei weiteren drei Patienten ein Röntgenbild an diesem Tag gemacht (Wirbelsäule, Becken). Bildgebende Diagnostik wie Computer- oder Magnetresonanztomographie wurde nicht eingesetzt.

\subsubsection{6 Ärztliche Anregungen}

40 Patienten $(82,5 \%)$ berichteten, dass sie von ihrem HA Tipps und Ratschläge für die häusliche Behandlung erhielten. An erster Stelle stand jede Art von sportlicher Bewegung (62,5\%) wie z.B. Rückenschwimmen, Fitness (besonders Kräftigungsübungen für die Rückenmuskulatur), Walken, Fahrradfahren und 
Gymnastik. Weiter wurde Wärme nach Belieben (Sauna, Wärmflasche, Bäder) empfohlen. Einhaltung einer korrekten Sitzhaltung, Vermeidung von Heben und Tragen schwerer Lasten wurden ebenso oft erwähnt. Bei vereinzelten Patienten $(n=2)$ wurde ein möglicher Zusammenhang der Rückenschmerzen mit psychischen Belastungen diskutiert. Bettruhe hingegen wurde kein einziges Mal „verordnet“. Abbildung 9 veranschaulicht die ärztlichen Empfehlungen im Überblick.

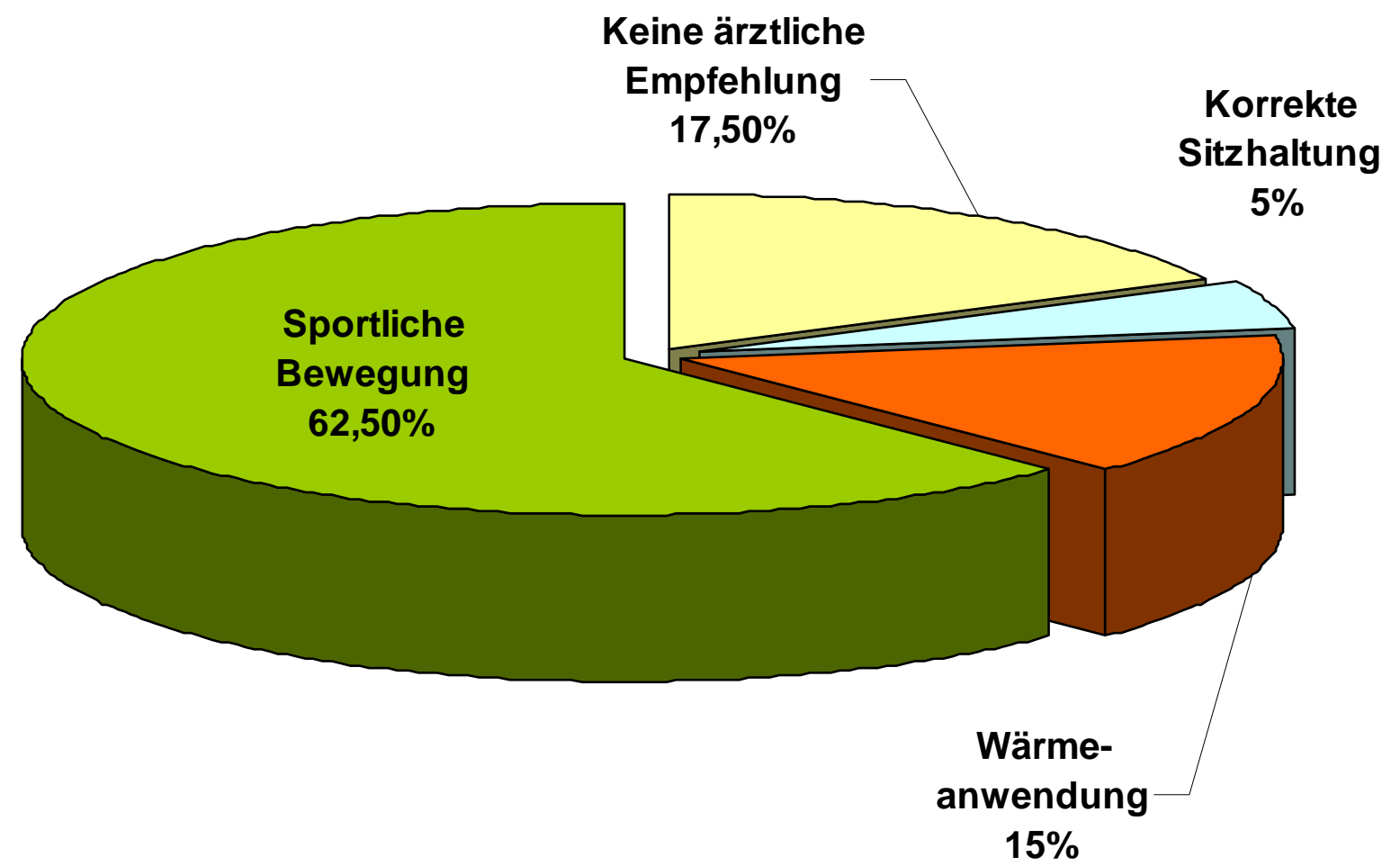

Abbildung 9: Ärztliche Anregungen $(n=40)$

\subsubsection{Ausstellen einer Arbeitsunfähigkeitsbescheinigung}

Fast einem Viertel $(22,4 \%)$ der Patienten wurde bei der Hausarztkonsultation eine AU-Bescheinigung ausgestellt, bzw. eine bereits vorhandene verlängert. 6 der insgesamt $11 \mathrm{AU}$ bekamen Patienten mit sehr starken Schmerzen (7-10). Weitere 3 
wurden an Patienten mit mittleren Schmerzen (4-6) und nur 2 an solche mit wenig Schmerzen ausgestellt. Einen graphischen Überblick dazu liefert Abbildung 10.

Von den 11 krank geschriebenen Patienten wurde bei 4 von ihnen die AU für 1 Woche ausgestellt, bei jeweils 2 weiteren Patienten für 1-4 Wochen und 4-8 Wochen. Ein Patient erhielt eine AU für länger als 8 Wochen. Eine schon bestehende Krankschreibung wegen Rückenschmerzen wurde bei zwei der Patienten auf über 6 Monate verlängert, wobei sich diese Patienten teilweise in Rehabilitations- bzw. Kurmaßnahmen nach einer Operation befanden.

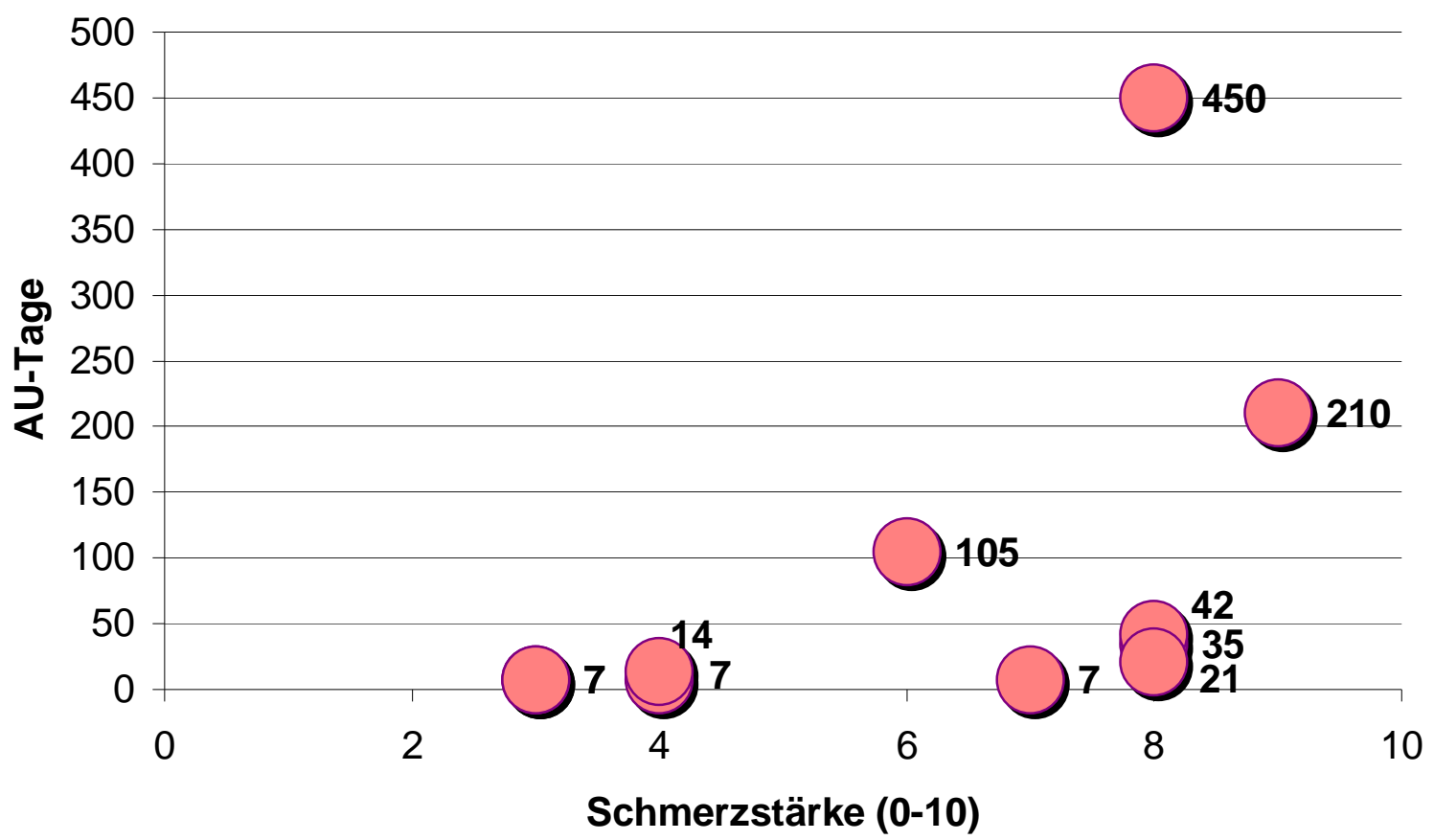

Abbildung 10: Arbeitsunfähigkeitstage von Patienten mit Kreuzschmerzen im Vergleich zur angegebenen Schmerzintensität am Tag der Hausarztkonsultation ( $n=11)$

\subsubsection{Rentenantrag}

Ein Patient (2\%) von den 49 Interviewteilnehmern berichtete, dass er am Tag seines Arztbesuches einen Rentenantrag gestellt hatte. Er war bis dahin berufstätig und ist 48 Jahre alt. Er war bereits seit über 450 Tagen krank geschrieben und gehört demnach zu den 18,2\% der Patienten, die schon länger als 6 Monate arbeitsunfähig sind. 


\subsubsection{Versorgungsleistungen im Follow-up $(n=40)$}

\subsubsection{Arztbesuch in den vergangenen 3 Monaten}

Die Prozentangaben in diesem Kapitel beziehen sich auf die 40 befragten Patienten, welche 12 Wochen nach dem Arztbesuch erneut telefonisch kontaktiert und über ihre Beschwerden befragt wurden. Die Daten zu den Unterpunkten 4.3.2.2 bis 4.3.2.4 wurden unabhängig von der Facharztrichtung (Allgemeinmediziner, Orthopäde oder Neurologe) in die Auswertung einbezogen.

28 Patienten suchten nach dem Rekrutierungszeitpunkt, also innerhalb von 3 Monaten, mindestens einmal erneut einen Arzt wegen Rückenschmerzen auf. Davon konsultierten 55\% ihren HA und ein Teil (10\%) davon zusätzlich einen Orthopäden und ein anderer Teil (5\%) einen Neurologen. 10\% berieten sich ausschließlich mit einem Orthopäden (5\%) oder einem Neurologen (5\%). Ein Patient (2,5\%) beratschlagte sich sowohl mit einem Orthopäden als auch einem Neurologen; ein anderer Patient (2,5\%) zog während einer Kur nur einen Kurarzt hinzu.

\subsubsection{Verschreibung von Medikamenten}

Bei den oben genannten Arztbesuchen wurden 45\% der Patienten $(n=40)$ Medikamente verordnet.

Die Ärzte verordneten am häufigsten NSAR (47,9\%), wobei erneut Diclofenac und Ibuprofen den Vorrang hatten. An zweiter Stelle standen hoch $(8,8 \%)$ und niedrig (13\%) potente Opioide. Andere zentral wirksame Medikamente gegen Muskel- und Skelettprobleme wie z.B. Tolperison wurden zu 17,4\% verschrieben. Unter den Medikamenten fanden sich auch Verordnungen von Cortison, Flupirtin und Antidepressiva (jeweils 4,3\%) wie sich in Abbildung 11 erkennen lässt. 


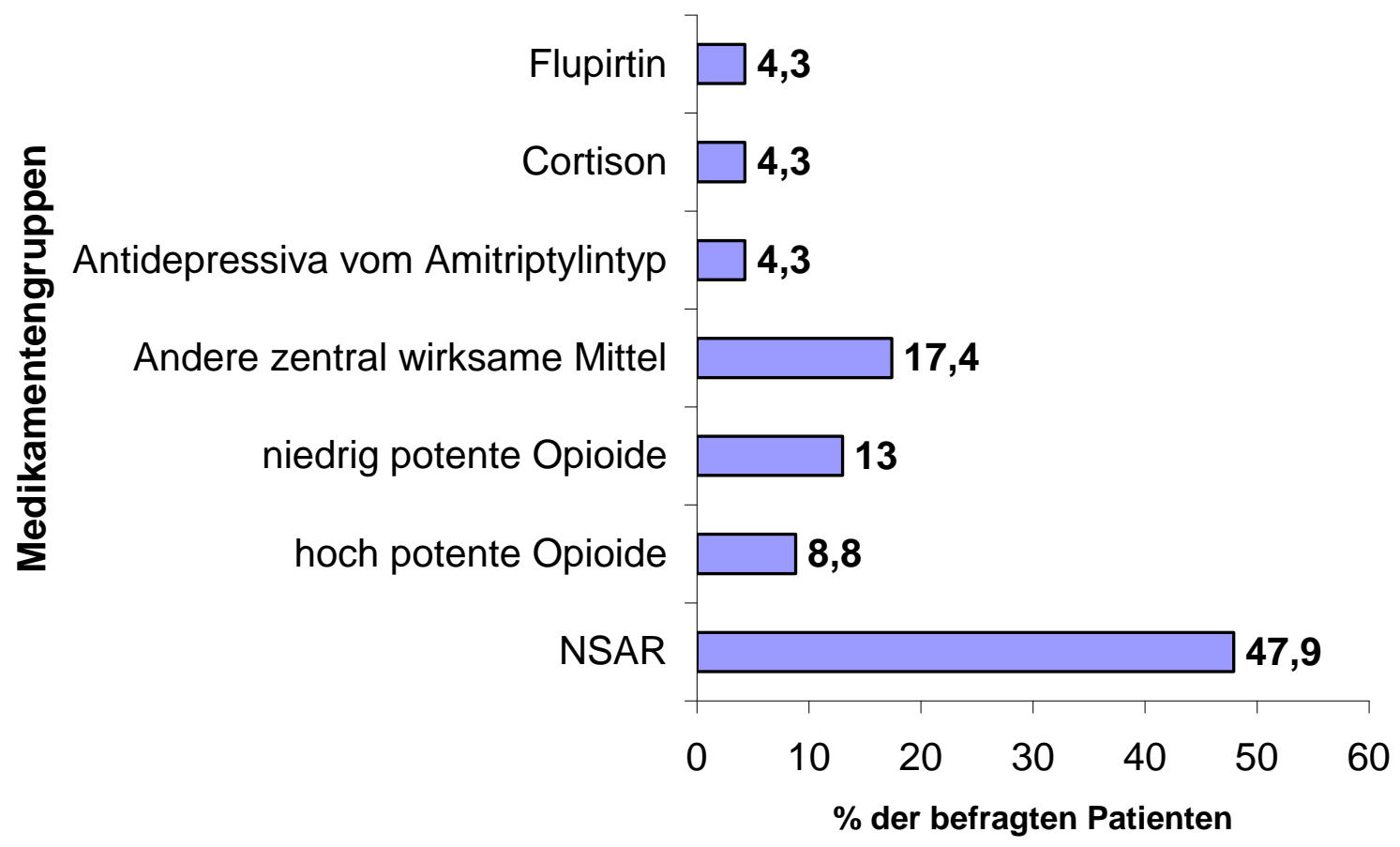

Abbildung 11: Verschreibung von Medikamenten im Follow-up von 3 Monaten

\subsubsection{Verabreichung von Injektionen}

Insgesamt erhielten mehr als zwei Drittel (37,5\%) der Patienten eine Spritze, wobei der Hauptteil (25\%) auf die intramuskuläre Injektion entfiel.

Tabelle 4: Gabe von Injektionen beim Arztbesuch in den letzten 12 Wochen $(n=40)$

\begin{tabular}{|c|c|}
\hline Art der Injektion & $\mathbf{n}(\%)$ \\
\hline intramuskuläre Spritze & $10(25 \%)$ \\
\hline paravertebrale Injektion & $3(7,5 \%)$ \\
\hline Quaddeln setzen & $2(5 \%)$ \\
\hline
\end{tabular}




\subsubsection{Durchführung weiterer Behandlungen}

Über der Hälfte der Patienten (52,5\%) wurde am Tag des Arztbesuches noch eine weitere Behandlung empfohlen, darunter vorwiegend Krankengymnastik (30\%) sowie Massagesitzungen (17,5\%).

Tabelle 5: Durchführung weiterer Behandlungen am Tag der Hausarztkonsultation

\begin{tabular}{|c|c|}
\hline Art der Behandlung & $\%$ \\
\hline Krankengymnastik & 30 \\
\hline Massage & 17,5 \\
\hline Akupunktur & 5 \\
\hline Keine Behandlung & 49 \\
\hline
\end{tabular}

\subsection{Krankheitsverlauf}

\subsubsection{Schmerzentwicklung}

Fast die Hälfte der Patienten (48\%), die einen FB in der Hausarztpraxis ausgefüllt hatte, gab am Tag der Hausarztkonsultation eine Schmerzintensität von $\geq 6$ (0 bedeutet keine Schmerzen, 10 bedeutet maximal denkbare Schmerzen) an (Tabelle 6). Zwei Wochen nach dem Arztbesuch teilten etwa ein Drittel (34,7\%) und 12 Wochen nur noch 27,5\% der Patienten mit, an Schmerzen der Stärke 6-10 zu leiden. Umgekehrt dazu gaben etwa die Hälfte der Patienten (52\%) zum Rekrutierungszeitpunkt an, nur an geringen bis mäßigen Schmerzen zu leiden, was auf der Skala einer vergleichbaren Stärke von 1-5 entsprach; es gab keinen Patienten, der nicht unter KS litt (Schmerzstärke 0 auf der Skala). Fast zwei Drittel $(65,3 \%)$ der Befragten hatten 2 Wochen und 72,5\% 12 Wochen später geringe bis mäßige Schmerzen (1-5). Schmerzfrei (0 auf der Skala) waren 2 Wochen nach dem Arztbesuch schon 8,1\% und 12 Wochen später 35\% der Befragten. Tabelle 6 zeigt 
diese Daten im Überblick; zu beachten ist die jeweils verschiedene Anzahl $\mathrm{n}$ der Patienten an den drei Befragungszeitpunkten ( $n=127, n=49$ und $n=40)$.

Tabelle 6: Kumulative Häufigkeiten der Angaben zur Schmerzstärke im Follow-up

\begin{tabular}{|c|c|c|c|}
\hline & Rekrutierungszeitpunkt & Nach 2 Wochen & Nach 12 Wochen \\
\hline Schmerzstärke & In \% (n= 127) & In \% (n = 49) & In \% (n = 40) \\
\hline $\mathbf{0}$ & 0 & 8,1 & 35 \\
\hline $\mathbf{1}$ & 0,8 & 20,4 & 40 \\
\hline $\mathbf{2}$ & 6,3 & 22,4 & 47,5 \\
\hline $\mathbf{3}$ & 18,9 & 38,8 & 62,5 \\
\hline $\mathbf{4}$ & 33,9 & 51 & 67,5 \\
\hline $\mathbf{5}$ & 52 & 65,3 & $\mathbf{7 2 , 5}$ \\
\hline $\mathbf{6}$ & 66,9 & 69,4 & $\mathbf{8 2 , 5}$ \\
\hline $\mathbf{7}$ & 83,4 & 87,8 & 90 \\
\hline $\mathbf{8}$ & 94,4 & 93,9 & 100 \\
\hline $\mathbf{9}$ & 96,8 & 100 & 95 \\
\hline $\mathbf{1 0}$ & 100 & 100 & 100 \\
\hline
\end{tabular}

\subsubsection{Entwicklung der Funktionalität}

Bei der Beantwortung dieser Frage sollten die Patienten beurteilen, ob sich ihre Beweglichkeit im Vergleich zu dem Zeitraum vor der Arztkonsultation nach 2 Wochen verbessert habe oder nicht (Abbildung 12). 2 Wochen nach dem Arztbesuch stellte sich bei mehr als zwei Drittel (69,6\%) der Patienten $(n=49)$ eine deutliche Verbesserung der Beweglichkeit ein (Frauen > Männer). 


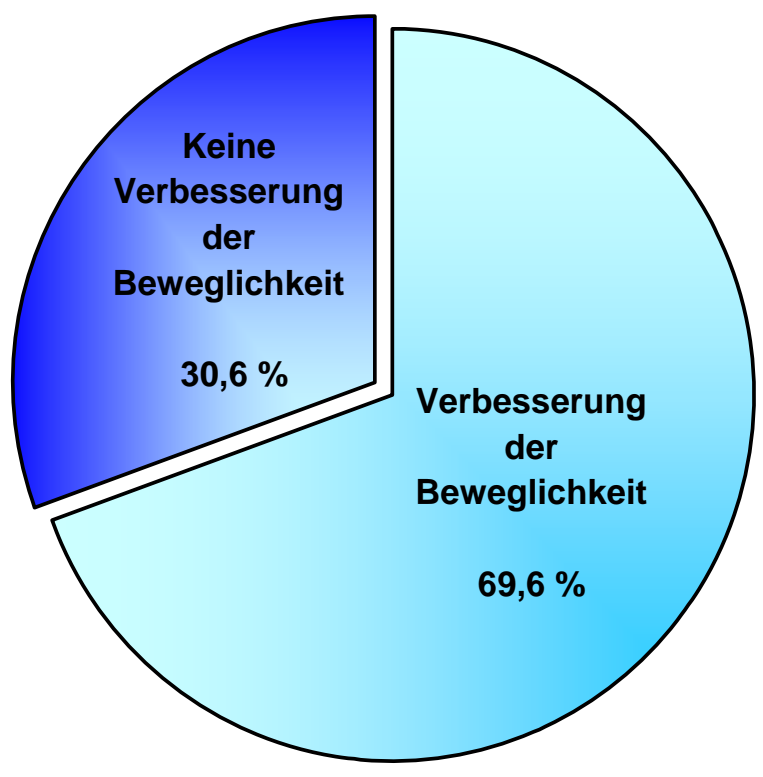

Abbildung 12: Verbesserung der Beweglichkeit $(n=49)$

Tabelle 7 demonstriert die funktionelle Beeinträchtigung in Tätigkeiten und Bedürfnissen, welche die Patienten aufgrund ihrer Schmerzen verspürten. 2 Wochen nach dem Arztbesuch fühlten sich $42,5 \%$ der Patienten gering ( 0 bis 3 auf der Numerischen Analogskala), 35\% mittelmäßig (4 bis 6 ) und $22 \%$ stark (7 bis 10 ) eingeschränkt. Nach 12 Wochen hingegen verspürten 17,5\% starke und 15\% Schmerzen mittlerer Stärke. Zwei Drittel der Befragten gaben geringe Einschränkungen (0 bis 3 ) in Tätigkeiten und Bedürfnissen des Alltags an. Der durchschnittliche Grad der Beeinträchtigung sank dementsprechend von durchschnittlich 4,4 nach 2 Wochen auf 2,7 (gering eingeschränkt) nach 12 Wochen.

Tabelle 7: Beeinträchtigung in Tätigkeiten und Bedürfnissen

\begin{tabular}{|c|c|c|c|c|c|c|c|c|c|c|c|}
\hline & $\mathbf{0}$ & $\mathbf{1}$ & $\mathbf{2}$ & $\mathbf{3}$ & $\mathbf{4}$ & $\mathbf{5}$ & $\mathbf{6}$ & $\mathbf{7}$ & $\mathbf{8}$ & $\mathbf{9}$ & $\mathbf{1 0}$ \\
\hline $\begin{array}{c}\text { nach 2 } \\
\text { Wochen(n=49) }\end{array}$ & 4 & 7 & 6 & 4 & 4 & 8 & 3 & 2 & 5 & 6 & 0 \\
\hline in \% & 8,2 & 14,3 & 12,3 & 8,2 & 8,2 & 16,3 & 6,1 & 4,1 & 7,2 & 12,3 & 0 \\
\hline $\begin{array}{c}\text { nach 12 } \\
\text { Wochen(n=40) }\end{array}$ & 16 & 2 & 5 & 4 & 3 & 2 & 1 & 3 & 1 & 3 & 0 \\
\hline in \% & 40 & 5 & 12,5 & 10 & 7,5 & 5 & 2,5 & 7,5 & 2,5 & 7,5 & 0 \\
\hline
\end{tabular}


Abbildung 13 zeigt die Werte der funktionellen Beeinträchtigung nach der zweiten Arztkonsultation (Vergleich nach 12 Wochen). Sie sind unabhängig von der angegebenen Schmerzintensität. Bei 62,5\% der Patienten sank der Grad der Beeinträchtigung, die Patienten empfanden ihre Schmerzen weniger als Hindernis im Alltag. Bei einem Viertel der Patienten verschlechterte sich die Lage hingegen, denn sie waren stärker eingeschränkt als 2 Wochen nach dem Arztbesuch. 12,5\% der Befragten stellten keinen Unterschied in ihrer funktionellen Beeinträchtigung fest. Es wurden nur die 40 Patienten eingeschlossen, die an beiden Telefonbefragungen (nach 2 Wochen, nach 12 Wochen) teilnahmen.

\section{Funktionelle Einschränkung in Tätigkeiten und Bedürfnissen...}

...gesunken

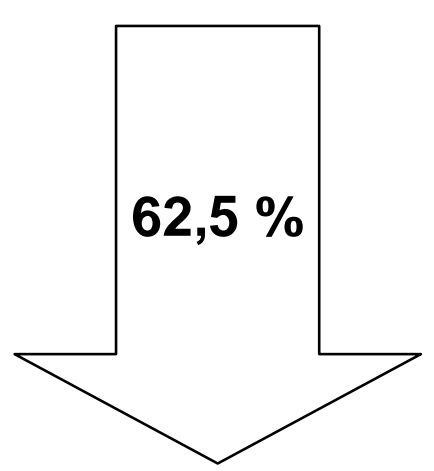

...gleich geblieben

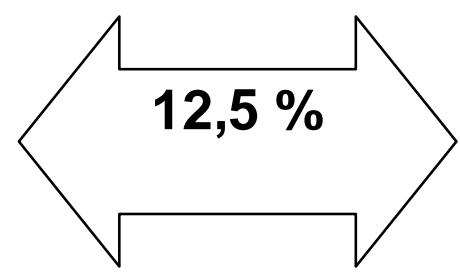

...gestiegen

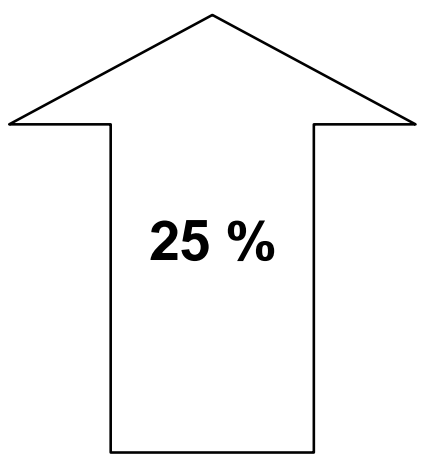

Abbildung 13: Vergleich der durchschnittlichen funktionellen Beeinträchtigung in Tätigkeiten und Bedürfnissen zwischen dem 1. und 2. Telefoninterview (Zeitraum 3 Monate; $n=40$ )

2 Wochen nach dem Arztbesuch fühlten sich mehr als vier Fünftel (81,8\%) der Befragten wohl bis sehr wohl (6 bis 10 auf der Numerischen Skala); 18,2\% fühlten sich nicht sehr gut bis schlecht (5 bis 0 auf der Skala).

12 Wochen nach dem Rekrutierungszeitpunkt berichteten zwei Drittel (67,5\%) über ein gutes bis sehr gutes Wohlbefinden (6-9 auf der Skala). Keiner der Rückenschmerzpatienten fühlte sich jedoch ausgezeichnet, was der Höchstpunktzahl 10 auf der Skala entspräche. Ein Zehntel der Befragten klagte über schlechtes bis sehr schlechtes Wohlbefinden (0 und 1 auf der Skala) wie aus der Tabelle 8 hervorgeht. Vergleichend kann zusammengefasst werden, dass sich der Großteil der Patienten 
sowohl 2 als auch 12 Wochen nach dem Arztbesuch gut bis sehr gut fühlte und ein kleineres Patientenkollektiv Unbehagen aufgrund der Schmerzen verspürte.

Tabelle 8: Wohlbefinden ( $n=40$ bzw. $n=49)$

\begin{tabular}{|c|c|c|c|c|c|c|c|c|c|c|c|}
\hline & $\mathbf{0}$ & $\mathbf{1}$ & $\mathbf{2}$ & $\mathbf{3}$ & $\mathbf{4}$ & $\mathbf{5}$ & $\mathbf{6}$ & $\mathbf{7}$ & $\mathbf{8}$ & $\mathbf{9}$ & $\mathbf{1 0}$ \\
\hline $\begin{array}{c}\text { nach 2 } \\
\text { Wochen (n=49) }\end{array}$ & 0 & 1 & 1 & 2 & 1 & 4 & 14 & 6 & 11 & 7 & 2 \\
\hline in \% & 0 & 2 & 2 & 4,1 & 2 & 8,2 & 28,6 & 12,3 & 22,4 & 14,3 & 4,1 \\
\hline $\begin{array}{c}\text { nach 12 } \\
\text { Wochen (n=40) }\end{array}$ & 2 & 2 & 0 & 1 & 4 & 4 & 7 & 7 & 9 & 4 & 0 \\
\hline in \% & 5 & 5 & 0 & 2,5 & 10 & 10 & 17,5 & 17,5 & 22,5 & 10 & 0 \\
\hline
\end{tabular}

Abschließend veranschaulicht Abbildung 14 noch einmal den Krankheitsverlauf mit Werten zur Schmerzintensität, Einschränkung durch die Schmerzen und dem Wohlbefinden.

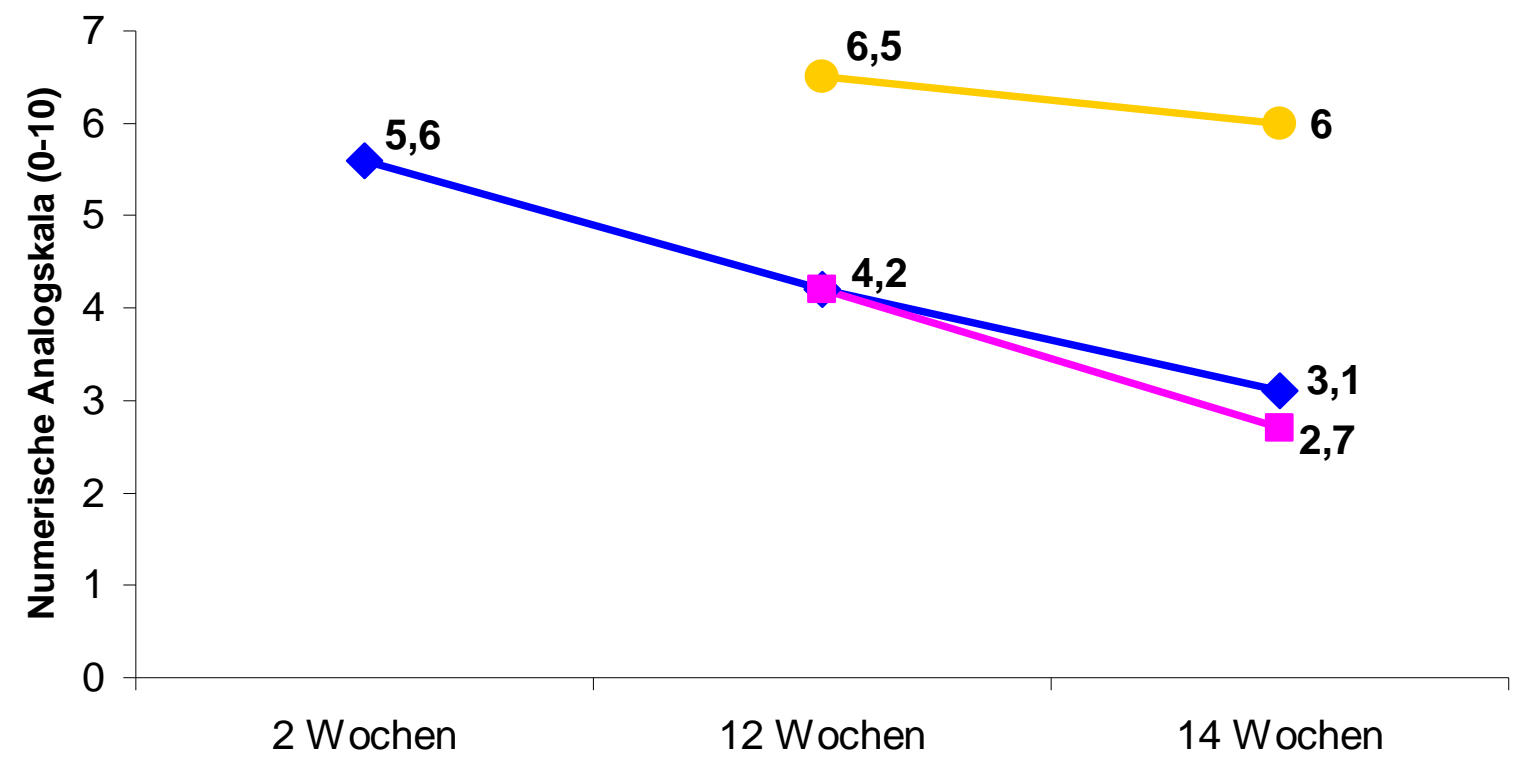

Zeitpunkt der Rekrutierung und follow-up

Schmerzstärke $\rightarrow-$ Einschränkung durch die Schmerzen - - Wohlbefinden

Abbildung 14: Vergleich der Parameter „Schmerzintensität“, „Einschränkung durch die Schmerzen“ und „Wohlbefinden“ im Krankheitsverlauf von 3 Monaten ( $n=40)$ 
Die durchschnittliche Schmerzintensität verringerte sich im Verlauf von 14 Wochen von anfangs 5,6 auf 3,1, was einem Abfall von 44,6\% entspricht. Auch die Einschränkungen der Patienten in ihren Tätigkeiten und Bedürfnissen reduzierte sich von 4,2 auf 2,7 (35,7\%). Das Wohlbefinden sank leicht um 7,7\% (von 6,5 auf 6).

\subsubsection{Subjektiver Krankheitsverlauf}

Über ein Drittel $(36,7 \%)$ der befragten Patienten meinten, dass sich ihre KS wieder bessern werden, also nur ein vorübergehender Schmerzzustand seien (Abbildung 15). Dagegen glaubten $63,3 \%$, dass keine Verbesserung mehr eintreten wird und sie sich für immer mit den Schmerzen auseinandersetzen müssen.

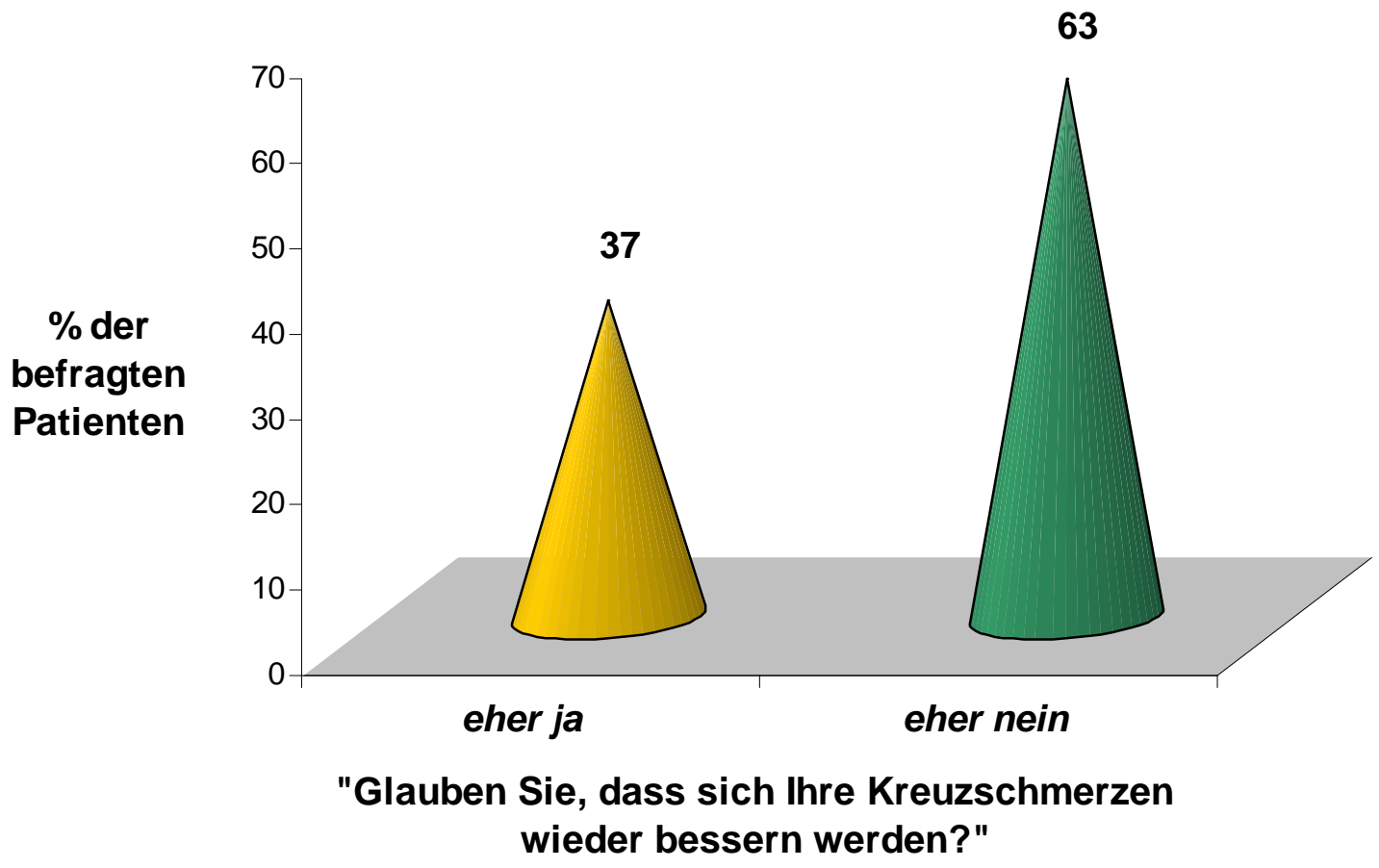

Abbildung 15: Subjektive Prognose aus Patientensicht über die Weiterentwicklung der Schmerzen $(n=49)$ 


\subsection{Patienteneinstellung}

\subsubsection{Selbstbewältigungsstrategien}

Bei dieser Frage konnten Patienten mehrere Möglichkeiten ankreuzen, so dass am Ende 276 Angaben 100\% gleichgestellt wurden. Insgesamt hatten 98\% der Patienten $(n=127)$ bereits vor dem Arztbesuch einen Versuch unternommen, ihre Schmerzen selbst in den Griff zu bekommen. An erster Stelle stand jede Art von Bewegung (30\%), z.B. Schwimmen, Radfahren, Fitness, Laufen und Gymnastik. Am zweithäufigsten wählten Patienten Schmerztabletten (24\%), gefolgt von der Wärmflasche (23\%). Für die Rubrik „Sonstiges“, wobei hier besonders Anwendungen wie Fangopackung, Körnerkissen, Akupunktur und TENS-Gerät genannt wurden, entschieden sich $12 \%$ der Patienten. Für Bettruhe entschieden sich $9 \% ; 2 \%$ unternahmen nichts gegen ihre Schmerzen.

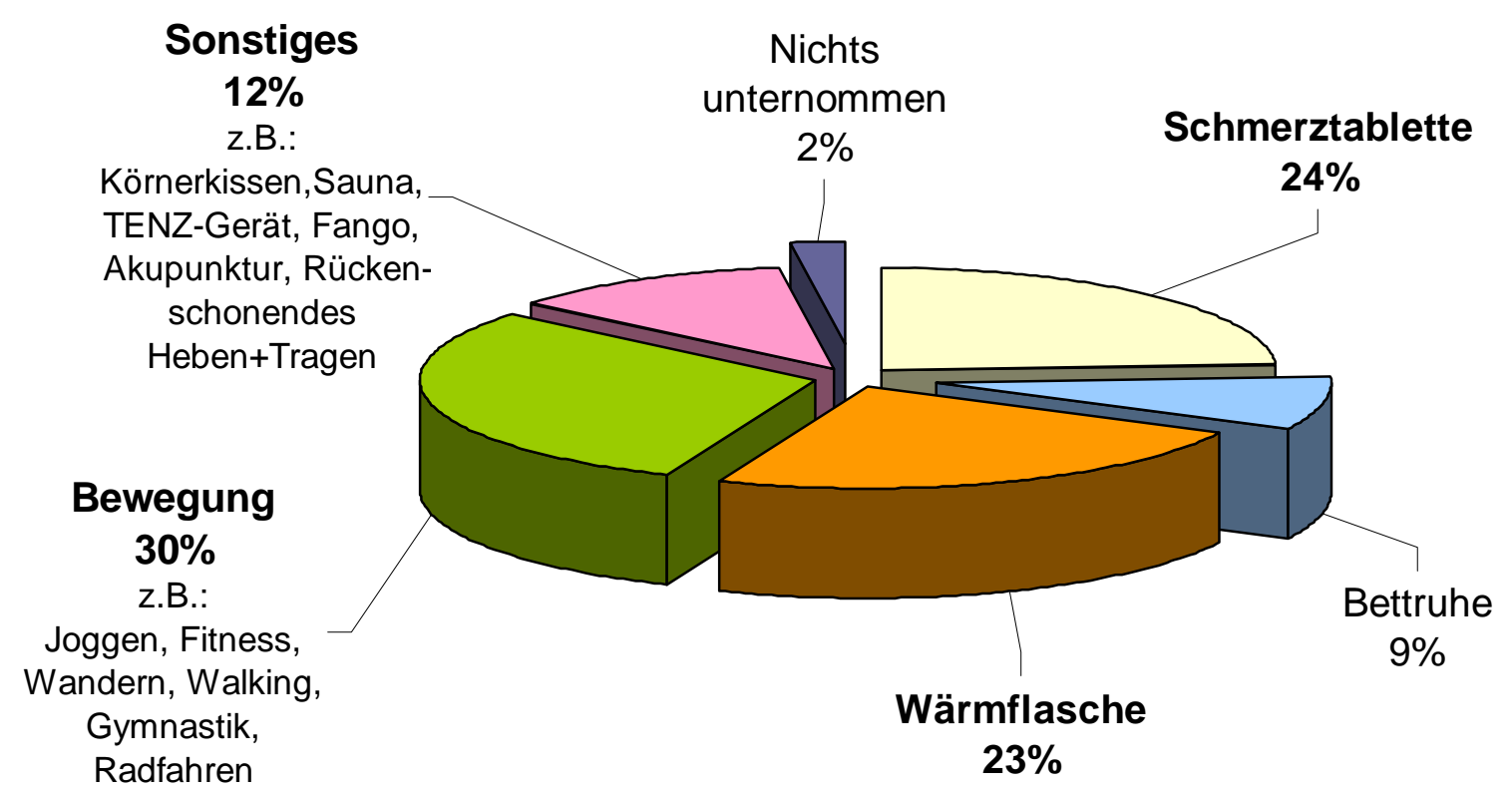

Abbildung 16: Selbstbewältigungsversuche der Patienten $(n=127)$, um Schmerzen zu lindern (276 Angaben insgesamt) 


\subsubsection{Selbsthilfe, außer durch Medikamente}

Die Tabelle 9 zeigt, wie gut Patienten ohne die Einnahme von Medikamenten Einfluss auf ihre Schmerzen nehmen konnten. 70\% der 49 Befragten arrangierten sich gut bis sehr gut (4 bis 10 auf der Skala) mit ihren Schmerzen. Im Freitext bestand die Möglichkeit, über die Art und Weise der Selbsthilfe Angaben zu machen. Erneut stand Bewegung als beste Alternative zu Medikamenten ganz oben.

Tabelle 9: Selbsthilfe, außer durch Medikamente („Wie gut konnten Sie heute, außer durch Medikamente, Einfluss auf Ihre Schmerzen nehmen?“; n=49)

\begin{tabular}{c|cccc} 
& 0 (gar nicht) & $\mathbf{1 - 3}$ (wenig) & 4- $\mathbf{6}$ (gut) & 7-10 (sehr gut) \\
\hline Gesamt $(\mathbf{n}=49)$ & $16 \%$ & $14 \%$ & $41 \%$ & $29 \%$
\end{tabular}

\subsubsection{Beste Behandlung der Schmerzen}

Von 127 Patienten hatten hier nur 76 (60\%) eine auswertbare Antwort, wie in der Fragestellung gefordert, angekreuzt. Die anderen 51 (40\%) konnten nicht berücksichtigt werden, weil sie mehrere Antworten gewählt hatten. Die Abbildung 17 zeigt, dass von 76 Patienten 22,8\% eine Spritze gegen ihre Schmerzen am sinnvollsten hielten, gefolgt von Krankengymnastik (17,7\%), Bewegung/ Sport $(16,5 \%)$ und Massage (16,4\%). Unter Sonstigem (8,9\%) erwähnten Patienten unter anderem „die Psyche heilen“, „manuelle Therapie“, „Reiten“, „Moorpackungen“ und „Tragen von Lasten nur mit Hilfsmitteln“. Wenige Patienten hielten Bettruhe $(7,6 \%)$, Schmerztabletten (6,4\%), Quaddeln setzen (2,6\%) und Einrenken (1,3\%) für die optimale Behandlung ihrer Beschwerden. 


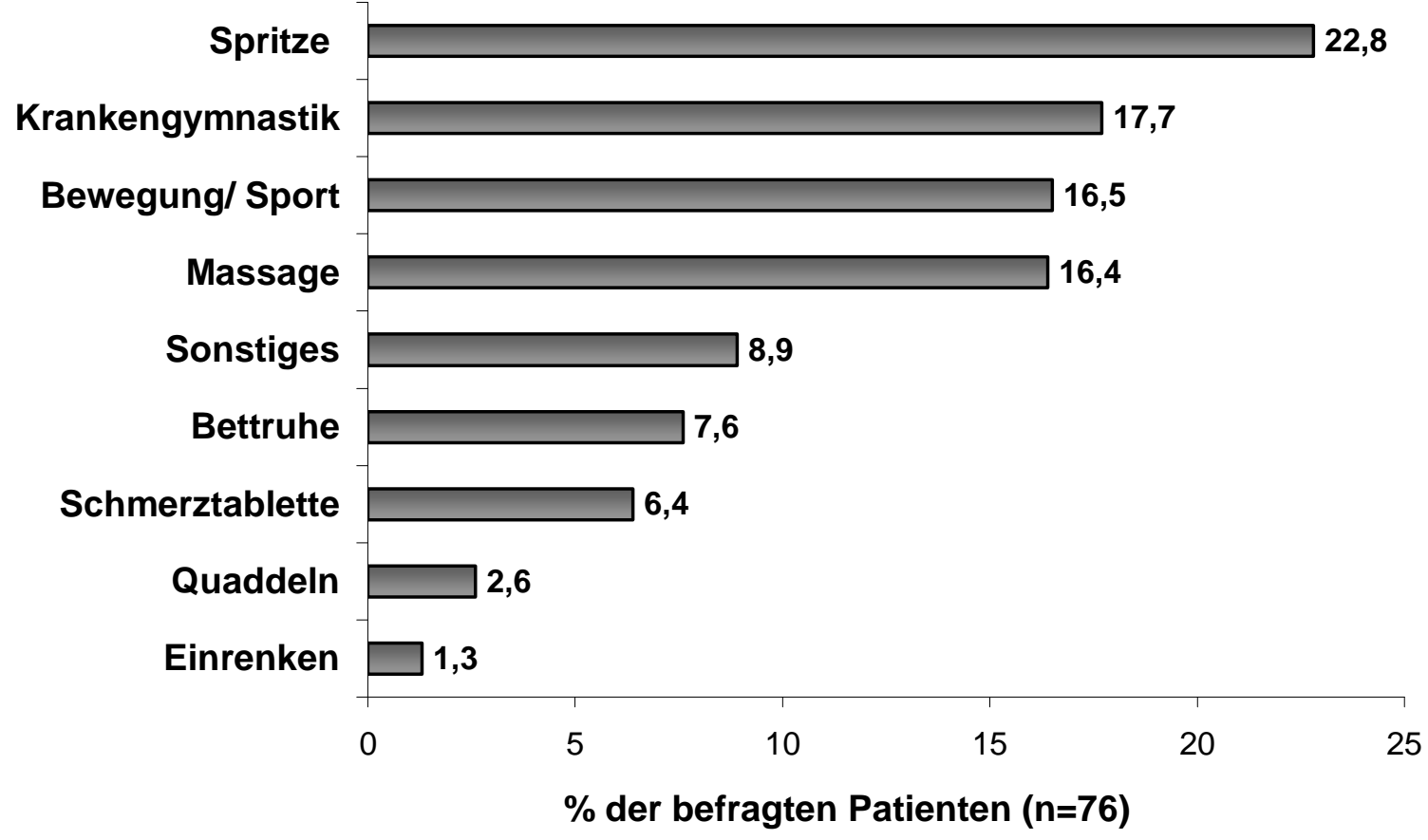

Abbildung 17: Beste Behandlung für die Beschwerden aus Patientensicht ( $n=76)$

Bei Vernachlässigung des Kriteriums der Einfachauswahl gehen 209 Angaben von 127 Patienten in die Bewertung ein. Hier standen an erster Stelle Massagen $(19,1 \%)$, gefolgt von Bewegung und Sport (18,6\%) und Spritzen gegen die Schmerzen $(17,7 \%)$.

\subsubsection{Wunsch nach weiterer Diagnostik}

29\% der befragten Patienten ( $n=127)$ wünschten sich betreffend ihrer Beschwerden weitere Untersuchungen (Computertomographie, Röntgen, betriebsärztliche Kontrollen). Dagegen hielten $71 \%$ der Befragten weitere Diagnostik für nicht sinnvoll. Einzelne gaben als Grund hierfür an, dass sie schon „durchdiagnostiziert" seien.

\subsubsection{Erwartungen des Patienten an die Arztkonsultation und Erfüllung dieser Erwartungen}

Abbildung 18 verdeutlicht, was für die Patienten $(n=110)$ am Tag der Hausarztkonsultation am Wichtigsten war (aus 5 vorgegebenen Antwortmöglichkeiten waren nur Einzelangaben erwünscht). Für $71,8 \%$ hatte die Behandlung der Schmerzen 
absolute Priorität. Das heißt, fast drei Viertel der Patienten, die den FB ausgefüllt hatten, suchten ihren HA auf, um ihre Schmerzen therapieren zu lassen. Einem weiteren Teil der Patienten (17,3\%) war es wichtig, mit ihrem Arzt über mögliche Optionen zu sprechen, wie sie ihre Schmerzen besser in den Griff bekommen könnten („Tipps und Ratschläge“). Eine Überweisung zu einem Facharzt und speziellere Untersuchungen wünschten sich 9,1\%. Mit dem besonderen Anliegen eines Kurantrags kamen immerhin noch 1,8\% zum HA. Dagegen bestand kein Interesse (0\%) am Erhalt einer Krankschreibung.

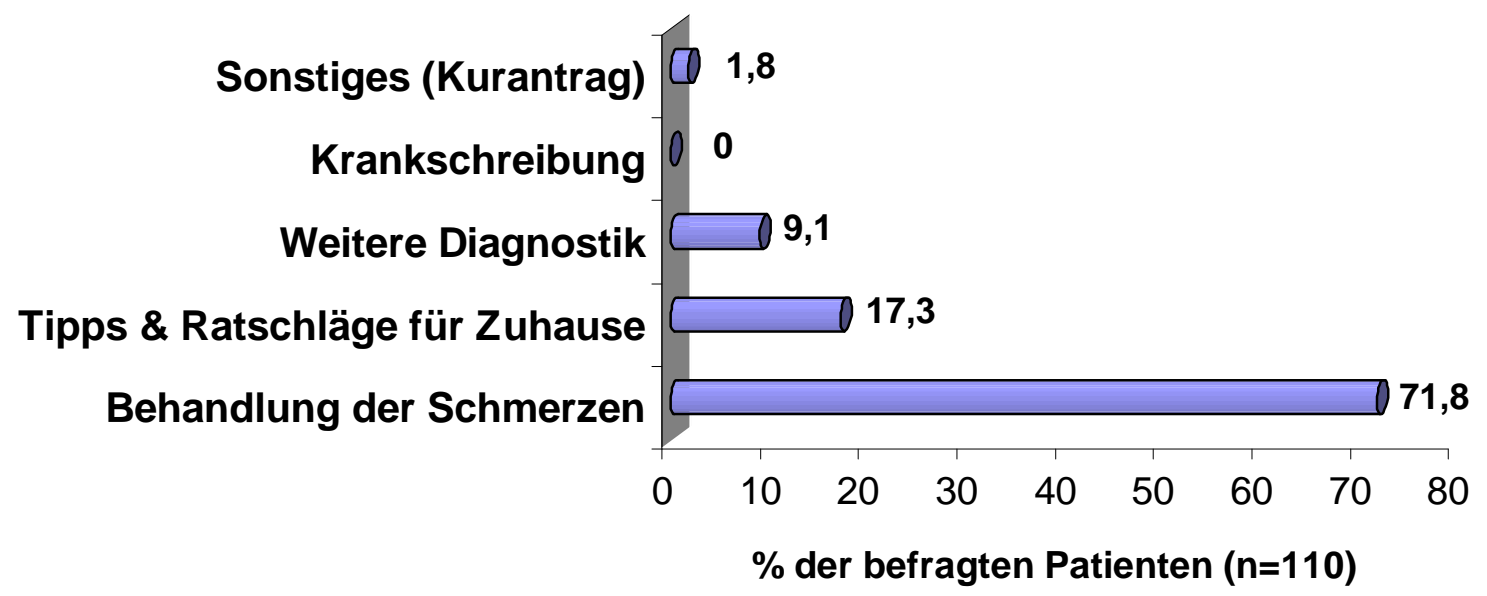

Abbildung 18: „Was ist für Sie bei Ihrem heutigen Hausarztbesuch am Wichtigsten?" $(n=110)$ 
Aus Tabelle 10 wird ersichtlich, ob sich die Erwartungen an den Hausarztbesuch erfüllten. Mit der Behandlung der Schmerzen waren 7 von 37 Patienten $(18,9 \%)$ und mit weiterer Diagnostik 1 von 4 Patienten (25\%) unzufrieden. Bei dem Großteil der Patienten erfültten sich die Erwartungen an den Hausarztbesuch: 81,1\% waren mit der Behandlung ihrer KS zufrieden und 100\% mit den ärztlichen Anregungen bezogen auf ihr Beschwerdebild.

Tabelle 10: Erfüllung der Erwartung an den Hausarztbesuch

\begin{tabular}{|c|c|c|}
\hline & Erfüllt & Nicht erfüllt \\
\hline Behandlung der Schmerzen $(n=37)$ & $30(81,1 \%)$ & $7(18,9 \%)$ \\
\hline Tipps und Ratschläge für Zuhause $(n=6)$ & $6(100 \%)$ & 0 \\
\hline Weitere Diagnostik $(n=4)$ & $3(75 \%)$ & $1(25 \%)$ \\
\hline
\end{tabular}




\subsubsection{Offene Fragen an den Arzt}

In offenen Antwortmöglichkeiten hatten die Patienten Gelegenheit, Fragen an ihren Arzt zu formulieren. Die Abbildung 19 zeigt schematisch die Häufigkeit der gestellten Fragen und deren Zuordnung zu Hauptschwerpunkten (in Klammern jeweils ein Beispiel eines Originalzitats der Patienten $(n=50)$.

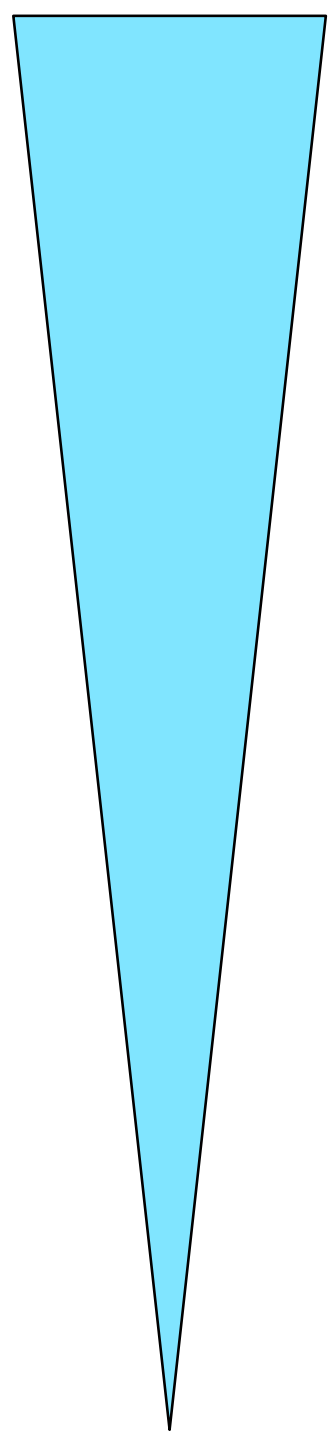

\section{Ursache der Schmerzen}

(„Woher kommen die Schmerzen?")

Therapiemöglichkeiten der Schmerzen

(„Was gibt es für Möglichkeiten, die Schmerzen zu lindern?“)

\section{Möglichkeiten zur Selbsttherapie}

(„Was kann ich selbst gegen die Schmerzen tun?")

\section{Prognose}

(„Wird man die Schmerzen je wieder los?“)

\section{Dauer der Schmerzen}

(„Wie lange halten meine Schmerzen noch an?")

\section{Prophylaxe}

(„Welche prophylaktischen Maßnahmen sind angebracht?“)

\section{Aktuelle Therapie}

(„Welche Behandlungsmethoden sind momentan notwendig?")

Abbildung 19: Häufig gestellte Fragen an den Hausarzt mit Originalzitaten von Patienten $(n=50)$ 


\subsubsection{Veränderungen der körperlichen Aktivität}

Im Freitext konnten sich die Patienten $(n=40)$ zu der Angabe „Veränderung der Aktivität" äußern. 21 der befragten Patienten (52,5\%) nutzten diese Gelegenheit. In der Abbildung 20 sind die Originalaussagen der Patienten kurz wiedergegeben.

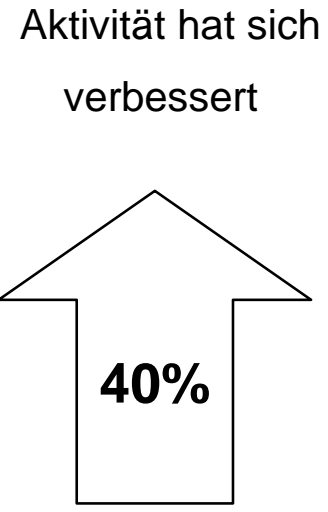

Aktivität gesteigert (40\%)
Aktivität ist gleich geblieben

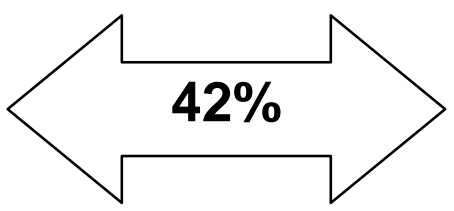

Aktivität hat sich verschlechtert

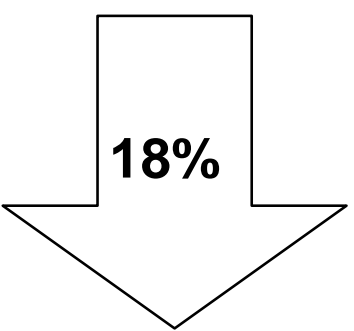

"Ich war mehr Schwimmen!" (n=3) "Ich bin mehr Rad gefahren!" ( $n=5)$ "Ich habe mehr Sport getrieben!" $(n=5)$ „Ich habe mehr Gymnastik für den Rücken gemacht!“ (n=3)

\section{Aktivität verringert (18\%)}

\author{
„Das Wetter macht so inaktiv!" (n=1) \\ „Mein rechter Arm ist gelähmt!“ ( $n=1)$ \\ „Bin nur noch ein psychisches Wrack!“ \\ $(n=1)$ \\ „Die Schmerzen machen mich \\ bewegungslos!" $(n=1)$ \\ „Bei mir ist nichts mehr möglich!" $(n=1)$
}

Abbildung 20: Veränderungen in der Aktivität in den letzten 12 Wochen $(n=21)$

40\% berichteten über eine Aktivitätssteigerung (Radfahren, Schwimmen, Fitness, Gymnastik), bei 42\% der Befragten veränderte sich nichts im Bereich der sportlichen Aktivitäten im Vergleich zur ersten Befragung vor 12 Wochen. Fast ein Fünftel (18\%) der Patienten fühlte sich durch die Schmerzen so stark eingeschränkt, dass diejenigen ihre körperlichen Aktivitäten verminderten. 
Aus der folgenden Tabelle 11 wird ersichtlich, dass 82,5\% aller befragten Patienten $(n=40)$ Empfehlungen durch ihren HA erhielten. Weit über der Hälfte der Patienten (62,5\%) empfahl der HA am Tag der Arztkonsultation Bewegung in Form sportlicher Aktivitäten. Andere Anregungen wie Wärmeanwendung (15\%) und auf eine korrekte Sitzhaltung zu achten (5\%) erwähnte der Arzt an zweiter bzw. dritter Stelle. Dagegen erhielten 17,5\% der Patienten von ihrem HA keine so genannten „Tipps und Ratschläge für Zuhause“ (Tabelle 10).

Tabelle 11: Empfehlungen der Ärzte zum Zeitpunkt der Rekrutierung $(n=40)$

\begin{tabular}{|c|c|c|c|c|}
\hline $\begin{array}{c}\text { Ärztliche Anregung } \\
\text { am Tag der Arzt- } \\
\text { konsultation }\end{array}$ & Bewegung & Wärme & $\begin{array}{c}\text { auf } \\
\text { Sitzhaltung } \\
\text { achten }\end{array}$ & $\begin{array}{c}\text { Keine } \\
\text { Empfehlung }\end{array}$ \\
\hline $\mathbf{n}=\mathbf{4 0}$ & $25(62,5 \%)$ & $6(15 \%)$ & $2(5 \%)$ & $7(17,5 \%)$ \\
\hline
\end{tabular}

Aus Tabelle 12 geht hervor, wie viele Patienten den hausärztlichen Ratschlag „Bewegung“ im Zeitraum nach der Arztkonsultation umsetzten. Fast drei Viertel (72\%) der Befragten waren sowohl 2 als auch 12 Wochen nach dem Arztbesuch körperlich aktiv, in Form von Radfahren, Schwimmen, Laufen und Gehen sowie Fitness, Gymnastik und Gartenarbeit. Meist betätigten sich die Patienten eine halbe bis eine Stunde. 28\% der Patienten waren nur an einem der beiden Zeitpunkte der Befragung sportlich aktiv. Favorisiert wurden in absteigender Reihenfolge Bewegungszeiträume zwischen 30 und 60 Minuten, 1 bis 30 Minuten, 60 bis 120 Minuten und mehr als 120 Minuten. 
Tabelle 12: Vergleich, inwieweit die Patienten, denen „Bewegung“ empfohlen wurde, den ärztlichen Rat annahmen $(n=25)$

\begin{tabular}{|c|c|c|}
\hline $\begin{array}{c}\text { Sportliche } \\
\text { Aktivitäten } \\
\text { ausgeführt... }\end{array}$ & $\begin{array}{c}\text {.. nach 2 und nach 12 } \\
\text { Wochen } \\
\mathbf{n = 1 8} \mathbf{( 7 2 \% )}\end{array}$ & $\begin{array}{c}\text {. an nur einem der } \\
\text { beiden Zeitpunkte } \\
\mathbf{n = 7} \mathbf{( 2 8 \% )}\end{array}$ \\
\hline $\begin{array}{c}\text { Favorisierte } \\
\text { Bewegungsform }\end{array}$ & $\begin{array}{c}\text { Radfahren, Laufen, } \\
\text { Schwimmen, Gehen, Fitness, } \\
\text { Gymnastik, Gartenarbeit }\end{array}$ & $\begin{array}{c}\text { Schwimmen, Walking, } \\
\text { Radfahren, Gymnastik }\end{array}$ \\
\hline
\end{tabular}

\subsubsection{Veränderungen im Alltag}

Eine weitere Übersicht über mögliche Veränderungen in den letzten 3 Monaten (nach der Hausarztkonsultation) den Alltag betreffend gibt Abbildung 21. Hier äußerten sich 17 von 40 Patienten (42,5\%).

\section{Aktive Maßnahmen ( $n=5)$}

- Mehr Bewegung über den Tag verteilt

- Entspannungsübungen, Gymnastik in den Tagesablauf eingebaut

\section{Passive Maßnahmen ( $n=12)$}

- Magnetstimulation bei der Krankengymnastik

- Akupunktursitzungen

- Kurantrag gestellt; zur Kur gefahren

- Körnerkissen gekauft; wärmendes Fell zugelegt

- Neue Matratze für das Bett gekauft

- Beim Heilpraktiker Spritzen auf pflanzlicher Basis bekommen

- Zur Psychotherapie angemeldet; Psychotherapie verlängert

- Wasserbett bestellt

Abbildung 21: „Was haben Sie selbst in den vergangenen 3 Monaten gegen Ihre Kreuzschmerzen unternommen (Veränderungen im Alltag)?" ( $n=17)$ 


\subsubsection{Krankheitsvorstellungen der Patienten}

Aus der Abbildung 22 geht hervor, welche Ursache für ihre KS die Patienten vermuteten. Diese Annahme muss nicht zwangsläufig die korrekte Diagnose sein.

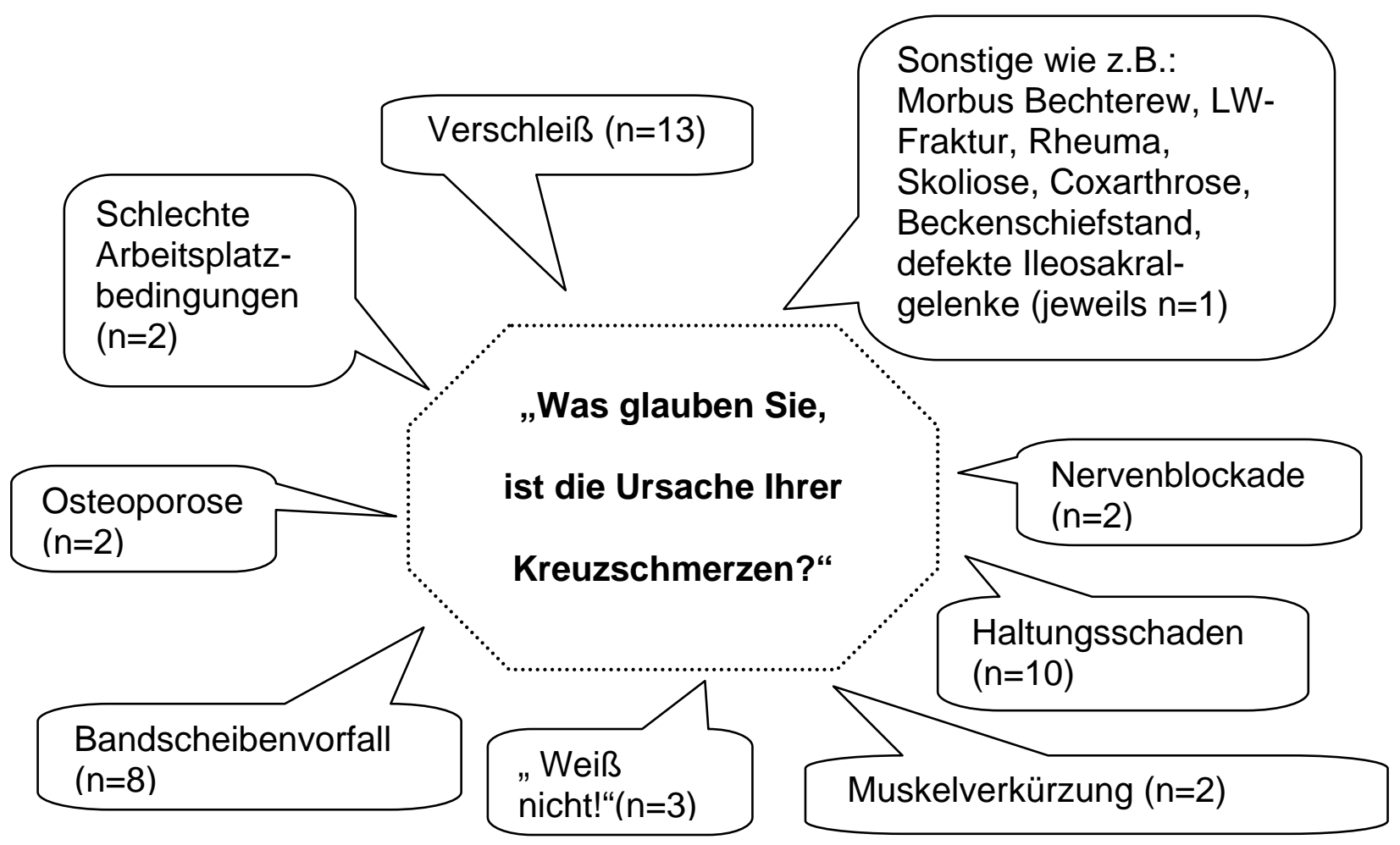

Abbildung 22: Ursache der Kreuzschmerzen aus Patientensicht $(n=49)$

Bei der Frage nach dem Einfluss der Stimmungslage auf die KS waren die Meinungen der Patienten ( $n=49)$ ausgeglichen. 47\% waren der Ansicht, dass die Stimmungslage nichts mit den Schmerzen zu tun hat. Die anderen 53\% der Befragten äußerten, dass die Stimmung keine Auswirkung auf die Schmerzen habe. 


\subsection{Pilotierung des Schmerztagebuches}

\subsubsection{Methodik}

Am Tag des zweiten Telefoninterviews wurden die Patienten gefragt, ob sie bereit wären, für 7 Tage ein Schmerztagebuch mit täglich 6 Fragen auszufüllen. Gab der Patient sein Einverständnis, wurden der Name und die vollständige Adresse notiert. Das Schmerztagebuch wurde innerhalb der nächsten Tage zugeschickt; es lag außerdem ein frankierter Rückumschlag bei. Die Telefonnummer der Abteilung Allgemeinmedizin stand für mögliche Fragen am Fußende jeder Seite zur Verfügung.

\section{Responsrate}

Von 40 Patienten erklärten sich 39 (97,5\%) am Telefon zur Teilnahme bereit. Es wurden demnach 39 Tagebücher verschickt. Davon wurden zwei Drittel vollständig zurückgesandt.

Es beteiligten sich 14 Männer und 12 Frauen. Das Durchschnittsalter $(n=24)$ lag bei 51,9 Jahren; 2 Patienten machten keine Altersangabe.

\section{Methodische Auswertung der Schmerztagebücher}

Alle 26 Tagebücher waren vollständig ausgefüllt. Es gab vereinzelt Probleme $(n=2)$ beim korrekten Ankreuzen der Zahlen auf der Numerischen Analogskala. Zum Beispiel wurden manchmal anstelle nur einer Zahl zwei Zahlen oder der Bindestrich zwischen zwei Zahlen angekreuzt.

\subsubsection{Ergebnisdarstellung}

In Tabelle 13 sind 4 von 7 Themen aus dem Schmerztagebuch dargestellt. Es wurde jeweils der Durchschnittswert von 7 Tagen berechnet $(n=26)$. Die Zahlenangaben beziehen sich auf verschiedene Numerische Analogskalen (0-10), die im Folgenden kurz erläutert werden: 
- Schmerzstärke: $\mathbf{0}=$ keine Schmerzen, $\mathbf{1 0}=$ maximal denkbare Schmerzen

- Einschränkung durch die Schmerzen: $\mathbf{0}=$ gar nicht beeinträchtigt, 10 = sehr stark eingeschränkt

- Einflussnahme auf die Schmerzen: $\mathbf{0}$ = gar nicht, $\mathbf{1 0}=$ sehr gut

- Wohlbefinden: $\mathbf{0}=$ sehr schlecht, $\mathbf{1 0}=$ sehr gut.

Tabelle 13: Übersicht der Durchschnittswerte von 4 Items aus dem Schmerztagebuch $(n=26)$

\begin{tabular}{|c|c|c|c|c|}
\hline & $\begin{array}{c}\text { Schmerz- } \\
\text { intensität }\end{array}$ & $\begin{array}{c}\text { Einschränkung } \\
\text { durch die } \\
\text { Schmerzen }\end{array}$ & $\begin{array}{c}\text { Einflussnahme } \\
\text { auf die } \\
\text { Schmerzen }\end{array}$ & Wohlbefinden \\
\hline $\operatorname{Tag}$ 1 & 4,2 & 3,6 & 3,7 & 5,7 \\
\hline $\operatorname{Tag}$ 2 & 4 & 3,2 & 3,2 & 5,9 \\
\hline $\operatorname{Tag} 3$ & 4,5 & 4,2 & 3,2 & 5,2 \\
\hline $\operatorname{Tag}$ 4 & 4,1 & 3,9 & 3,7 & 5,3 \\
\hline $\operatorname{Tag} 5$ & 4,3 & 3,7 & 3,3 & 5,3 \\
\hline $\operatorname{Tag} 6$ & 4 & 3,8 & 4,2 & 5,7 \\
\hline Tag 7 & 3,8 & 3,5 & 4,2 & 6 \\
\hline $\begin{array}{c}\text { Gesamt } \\
\mathbf{n = 2 6}\end{array}$ & $\mathbf{4 , 1}$ & $\mathbf{3 , 7}$ & $\mathbf{3 , 6}$ & $\mathbf{5 , 6}$ \\
\hline
\end{tabular}

Die durchschnittliche Schmerzstärke betrug 4,1 (0,6-6,9). In ihren alltäglichen Tätigkeiten und Bedürfnissen fühlten sich die Patienten mäßig durch ihre KS (3,7 [07,1]) eingeschränkt. Den Punkt der Einflussnahme auf die Schmerzen außer durch Medikamente betrachtend, fällt ein Wert von durchschnittlich 3,6 [3-7,6] auf. Der Durchschnittswert für das Wohlbefinden lag bei 5,6 (3-7,6) auf der Skala. 
Abbildung 23 veranschaulicht nun vergleichend die o.g. Ergebnisse der Auswertung des Schmerztagebuches mit den durchschnittlichen Resultaten des 1. Telefoninterviews nach 2 Wochen $(n=26)$.

Die Schmerzstärke betreffend gab es kaum Veränderungen die beiden Zeitpunkte vergleichend $(4,2 \rightarrow 4,1)$. Die Patienten fühlten sich weniger durch die Schmerzen eingeschränkt $(4,9 \rightarrow 3,7)$. Sie konnten schlechter Einfluss auf ihre Beschwerden nehmen $(4,9 \rightarrow 3,6)$ und gaben niedrigere Werte für ihr Wohlbefinden an $(6,5 \rightarrow 5,6)$.

\section{Vergleich der Daten 2 Wochen (1.Tel.) und 14 Wochen (ST) nach der Arztkonsultation}

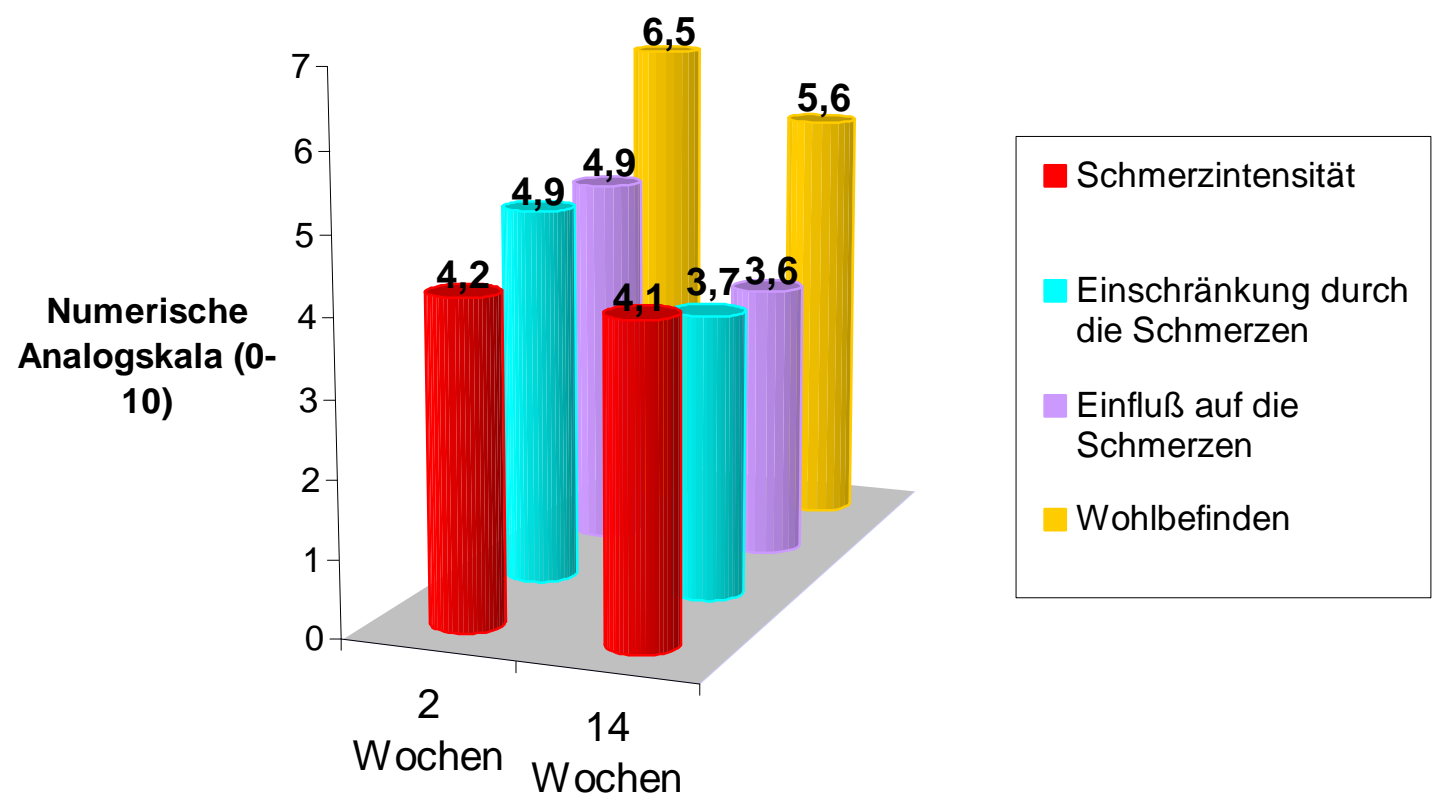

Abbildung 23: Vergleich der Daten für "Schmerzintensität”, „Einschränkung durch die Schmerzen“, „Einflussnahme auf die Schmerzen“ und „Wohlbefinden“ 2 Wochen (1. Telefoninterview) und 14 Wochen (Schmerztagebuch) nach der Arztkonsultation $(n=26)$

\section{Einnahme von Schmerzmedikamenten}

Über die Hälfte $(57,7 \%)$ der befragten Patienten $(n=26)$ nahmen aufgrund der KS an mindestens einem der 7 Tage Medikamente ein. Dabei griff ein Drittel (34,6\%) an 1 bis 4 Tagen und 23,1\% an 5 bis 7 Tagen auf Schmerzmittel zurück. 42,3\% benötigten keine Schmerzmedikation. 
Am häufigsten nannten die Patienten NSAR wie z.B. Diclofenac, Paracetamol, Ibuprofen ( $n=10)$, Muskelrelaxantien (z.B. Mydocalm, n=2), Analgetika (z.B. Tramal, $\mathrm{n}=2$ ) und Antidepressiva (z.B. Saroten, $n=1)$.

\section{Arztbesuche}

Insgesamt suchten 6 Patienten (23,7\%) einmal ihren HA und 3 Patienten $(11,5 \%)$ einmal einen Orthopäden innerhalb der 7 Tage auf.

\section{Körperliche Aktivität}

In den 7 Tagen betätigten sich 24 von 26 Patienten (92,3\%) durchschnittlich 5 Mal pro Woche sportlich aktiv (3-7). Minutenangaben variierten zwischen 1 bis $29(n=9)$, 30 bis $59(n=6), 60$ bis $89(n=5), 90$ bis $120(n=3)$ und mehr als 120 Minuten Sport am Tag $(n=1)$. Beliebt war auch hier wieder das Radfahren, Schwimmen, Fitness, Laufen, Gehen sowie Gymnastik für den Rücken. Einige zogen Entspannungsübungen oder Bewegungsbäder vor.

2 Wochen nach der Arztkonsultation trieben 25 der 26 Patienten (96,1\%) Sport, also geringfügig mehr. Gehen, Radfahren, Laufen sowie Gymnastik und Schwimmen standen schon zu diesem Zeitpunkt ganz oben auf der Liste der Betätigungen. Die Werte können nicht direkt verglichen werden, da am Tag des Interviews nach der sportlichen Aktivität des Tages gefragt wurde und von den 24 Patienten, die hierzu Angaben im Schmerztagebuch machten, nicht alle gleichmäßig oft aktiv waren einige nur 3, andere $7 \mathrm{Mal}$ pro Woche. 


\section{Diskussion}

\subsection{Hauptaussage der Studie}

Rückenschmerzen sind ein bedeutendes Gesundheitsproblem industrialisierter Länder. Trotz hoher Spontanheilungstendenz führen häufige Rezidive und chronische Verläufe zu langen Arbeitsunfähigkeitszeiten und Frühberentungen. Besondere Bedeutung für die Prävention solcher Fälle kommt den HÄ zu. Trotzdem ist über die Häufigkeit von Konsultationen wegen Rückenschmerzen und deren Versorgung in deutschen Hausarztpraxen bislang wenig bekannt.

Die Studie gewährt einen Einblick in die Epidemiologie, den Krankheitsverlauf und die Versorgung von Kreuzschmerzpatienten in der hausärztlichen Praxis. Zudem sind die Wünsche und Erwartungen dieser Patienten an den konsultierten Arzt untersucht worden.

Ein hoher Anteil der Patienten in der Praxis hatte nicht akute, sondern schon lang bestehende Schmerzen (fast ein Drittel hatte in den letzten 3 Monaten täglich Schmerzen, teilweise mit Rezidivcharakter). Die von der Kreuzschmerz-Leitlinie der DEGAM (Becker et al. 2003) propagierte Vorgehensweise von Schmerztabletteneinnahme und Rat zu mehr Bewegung haben schon 24 bzw. 30\% der Patienten versucht. Sie kommen mit dem Wunsch nach anderen Therapiemethoden (hauptsächlich „Spritze gegen die Schmerzen“, „Krankengymnastik“, „Massage“) zum HA. Das Therapiespektrum der Ärzte ist weit. Häufig werden evidenzbasierte Maßnahmen (60\% NSAR-Verordnungen) bzw. ein aktivierender Behandlungsansatz eingesetzt. Allerdings wird oftmals auch auf Therapiemethoden geringer oder fehlender Evidenz (42,9\% Injektionstherapien, 8,2\% Massagen) zurückgegriffen.

\subsection{Vergleich der Studienergebnisse mit internationaler Literatur}

Evidenzbasierte Leitlinien wurden in verschiedenen Ländern erstellt, um die Versorgung von Rückenschmerzpatienten zu rationalisieren. Die Empfehlungen sind häufig ähnlich. Koes et al. (2001) stellten in einem Vergleich europäischer Leitlinien Gemeinsamkeiten bezüglich diagnostischer und therapeutischer Empfehlungen 
(frühe Wiederaufnahme der Aktivitäten der Patienten, Meiden von Bettruhe, Erhebung von Risikofaktoren für eine Chronifizierung) fest. In den meisten Ländern sind Neuauflagen der Leitlinien geplant bzw. bereits realisiert (Koes et al. 2001). Für die Deutsche Allgemeinmedizin ist die 2003 veröffentlichte DEGAM-Leitlinie (Becker et al.) zur bedarfsorientierten und qualitativen Primärversorgung von Patienten mit $\mathrm{KS}$ von Bedeutung.

Zahlreiche Studien befassen sich mit der Leitlinienadhärenz verschiedener Ärztegruppen. So zeigte eine in Italien veröffentlichte Studie (Negrini et al. 2001), dass nur 28\% der Allgemeinmediziner die diagnostischen Tests durchführen, die die klinischen Leitlinien für wichtig halten, und nur ein Drittel sich der Warnzeichen („red flags“) bewusst ist. Einer der Gründe, weshalb HÄ sich nicht an die Leitlinien halten, ist die von ihnen wahrgenommene Vorliebe der Patienten für bestimmte Behandlungen, die meist auf früheren medizinischen Erfahrungen beruhen (Schers et al. 2000).

Zum Rekrutierungszeitpunkt und im Follow-up erhielten 42 bzw. 45\% der Befragten ein orales Schmerzmittel. 60\% aller Verordnungen entfielen auf NSAR, z.B. häufig Ibuprofen und Diclofenac. 40\% erhielten vom Arzt eine Injektion, davon mehr als zwei Drittel intramuskulär. Diese Entscheidung für eine obsolete Therapie (Becker 2003) könnte im Zusammenhang mit den Wünschen der betroffenen Patienten stehen (22,8\% halten „eine Spritze gegen die Schmerzen“ am sinnvollsten), welches die o.g. Vermutung Schers unterstreicht. Die hohe Zahl (> 50\%) an Empfehlungen bzw. Verordnungen von kontrovers diskutierten, nicht medikamentösen Behandlungen (wie z.B. Krankengymnastik, Kurzwelle, Massage, Akupunktur) fällt auf. Auch hier liegt die Vermutung nahe, dass die $\mathrm{HÄ}$ oft den Erwartungen der Patienten nachkommen möchten. Die Auswertung der Patientenwünsche deckte eine ebenso hohe Anzahl dieser Behandlungsmethoden auf. Die Mehrheit der Allgemeinmediziner akzeptiert die Eigenständigkeit der Patienten bezüglich der Nutzung alternativer Therapieverfahren (Rogers 2002) und zudem könnten sich die Ergebnisse verbessern, wenn die Patienten die Behandlung wählen, die ihrer Meinung nach am besten hilft (Cherkin et al. 2003). Kein Patient erhielt eine Überweisung zum Psychotherapeuten, obwohl psychosoziale Faktoren in der Chronifizierung von KS nachweislich eine Rolle spielen. So sind beispielsweise depressive Verstimmungen, private und berufliche Unzufriedenheiten und ein 
ungünstiger Krankheitsverlauf mit einer höheren Rate an Chronifizierungen assoziiert (Hasenbring 1992). Das Fehlen entsprechender Überweisungen kann einerseits auf das ungenügende Wissen der $\mathrm{HÄ}$ um einen solchen Zusammenhang bzw. die mangelhafte Umsetzung der Therapieempfehlungen zurückzuführen sein, oder aber ein Ausdruck fehlender Therapieplätze.

Eine Arbeitsunfähigkeitsbescheinigung am Tag der Konsultation erhielten 22\% der befragten Patienten. Die Ergebnisse der Studie zeigen, dass Patienten mindestens eine Woche oder länger krankgeschrieben wurden. Göbel (2001) berichtet zusammenfassend aus Studien Ende der 80er Jahre, dass 16 Millionen Arbeitsunfähigkeitstage pro Jahr induziert und somit 4\% der gesamten Arbeitskraft in Deutschland ausgeschaltet worden sind. Nach den Leitlinienempfehlungen sollte die Arbeit möglichst nach 2-3 Tagen wieder aufgenommen werden (Becker et al. 2003).

Es lässt sich feststellen, dass sich die Diskrepanz zwischen Leitlinienempfehlungen und dem tatsächlichen Verhalten der Allgemeinmediziner weiter verringert, so wie es Frankel et al. über die Dauer von 5 Jahren in England beobachtete. Er und sein Team konnten im Zeitraum von 1992-97 feststellen, dass Allgemeinmediziner nach fünf Jahren mehr für körperliche Aktivität für Kreuzschmerzpatienten plädierten besonders für jüngere Menschen - als zu Beginn der Erhebung. Betroffene Patienten suchten ihren HA zu einem früheren Zeitraum als sonst auf, was für wenig Selbständigkeit seitens des Patienten spricht und/oder eine hohe Erwartungshaltung an den HA für entsprechende Hilfe bedeutet (Frankel et al. 1999).

Einer der Gründe nicht leitliniengerechter Tätigkeit von $\mathrm{HÄ}$ ist der, dass entsprechende Patientenerwartungen angenommen werden. Seitens der Patienten verneint mehr als die Hälfte einen Einfluss der Stimmungslage auf den Grad der Beschwerden und ist stattdessen von somatischen Erkrankungen als Ursache der KS überzeugt. Aufgrund der Beeinträchtigung im Alltag und der Einschränkungen bei täglichen Verrichtungen haben die meisten Patienten (98\%) selbst etwas unternommen, um ihre Schmerzen in den Griff zu bekommen. Erfreulicherweise rangieren aktive Bewegungsformen ganz oben, auch nach 3 Monaten, gefolgt von Wärmeanwendungen. Die Bekräftigung dieser Tatsache ist die häufig genannte Anregung der $\mathrm{HÄ}$, sich aktiv zu bewegen $(62,5 \%)$ und keinesfalls zu ruhen (Bettruhe 
0\%). Frühzeitige Mobilisierung wird von evidenzbasierten Leitlinien national und international empfohlen (Becker et al. 2003, Koes et al. 2001, Schers et al. 2000, Frankel et al. 1999). Weiterhin auffallend ist der geringe Anteil an Patienten, die sich weitere Diagnostik als Behandlungsziel wünschen. Die Mehrheit $(71,8 \%)$ stuft die Behandlung ihrer KS beim Hausarztbesuch als oberste Priorität ein. Diese Entwicklung (frühzeitige Aktivierung, Verzicht auf Diagnostik und konsequente Schmerztherapie) propagieren alle gegenwärtigen Leitlinien (Becker et al. 2003, Koes et al. 2001, Frankel et al. 1999). Southgate und Bass (1983) konnten zeigen, dass die vorrangige Erwartung der Patienten der verständlichen Erläuterung der Diagnose und Therapie durch den HA und einer freundlichen und verständnisvollen Umgangsart miteinander gilt. Einige Ergebnisse dieser Studie zeigen dazu Parallelen. Zum Beispiel haben Patienten die ärztlichen Anregungen bzw. Tipps und Ratschläge angenommen und waren in über $80 \%$ der Fälle mit der Behandlung ihrer Schmerzen zufrieden. Vor dem Arztbesuch wünschten sich 9,1\% der Patienten weitere Aufklärung ihrer KS. Diese Erwartung wurde bei $75 \%$ der befragten Patienten (Telefoninterview) erfüllt. Kochen (1999) zeigte, dass Patientenerwartungen zum Ergebnis der medizinischen Konsultation sich nicht nur auf die Heilung oder Befreiung von Beschwerden beziehen, sondern auch das Bedürfnis nach Kommunikation, Information und Einfühlungsvermögen widerspiegeln. Zudem ist die Übereinstimmung zwischen Arzt und Patient betreffend der Diagnose, der diagnostischen und therapeutischen Vorgehensweise mit einer höheren Patientenzufriedenheit und einem besseren Gesundheitszustand assoziiert (Staiger et al. 2005). Rogers untersuchte in Australien (2002) die Einstellung von HÄ gegenüber Patientenautonomie in der Behandlung von KS. Die Mehrheit der Allgemeinmediziner akzeptierte die Eigenständigkeit der Patienten bezüglich der Nutzung alternativer Behandlungsverfahren, kontrollierte aber die Einnahme von Analgetika gründlich. Die Ärzte hatten bezüglich der Dauer der Arbeitsunfähigkeitszeiten eine andere Meinung als ihre Patienten, dagegen zeigten ihre Einstellungen zum Gebrauch von Röntgendiagnostik eine Assoziation mit den Forderungen der Patienten nach radiologischer Zusatzdiagnostik.

Letztlich könnte die Vielfalt der Therapiemethoden auch eine gewisse Hilflosigkeit der Ärzte widerspiegeln, da viele Empfehlungen der Leitlinie (Einsatz von NSAR, Aktivität) schon umgesetzt wurden. Das Gros der Patienten in der Allgemeinarztpraxis hat rezidivierende und langandauernde Beschwerden, die den 
Arzt möglicherweise in Handlungsnot bringen, da evidenzbasierte Verfahren bereits eingesetzt wurden, nicht realisierbar sind oder nicht mehr ausreichen.

Die epidemiologischen Ergebnisse dieser Studie entsprechen ähnlichen Erhebungen international. Es wurde hier eine 1-Wochen-Inzidenz von 5,5\% festgestellt. Diese Daten spiegeln internationale Ergebnisse wie z.B. eine kumulative Jahresinzidenz von 6,4\% in Großbritannien durchaus wider (Croft et al. 1998). Freeborn et al. (1997) fanden in einer Arbeit von 1987 eine jährliche Inzidenzrate von 6-7\% für KS unter allen Erwachsenen. Fast 20\% der Patienten beklagten ein Rezidiv nach einem symptomfreien Intervall von 6 Monaten. Die Ergebnisse der vorliegenden Studie zeigten nach 3 Monaten komplette Schmerzfreiheit bei 8\% der Patienten. 37\% der Patienten gaben an, an vereinzelten Tagen KS zu spüren. Mehr als die Hälfte der Patienten litt häufig (einmal wöchentlich) bis täglich an Beschwerden. Dies steht im Einklang mit der oft beschriebenen Tatsache, dass sich Patienten mit KS innerhalb weniger Wochen von ihren Beschwerden erholen und funktionelle Besserung spüren, nichtsdestotrotz aber Schmerzen und Einschränkungen wiederkehren (Pengel et al. 2003).

\subsection{Beurteilung der Methode}

Es handelt es sich um eine Querschnittserhebung mit anschließender prospektiver Kohortenstudie über 3 Monate. Zur Erleichterung der Durchführung der Studie wurden Allgemeinmediziner in einem Landkreis (Südoldenburg) angeschrieben; dieses spiegelt einen Selektionseffekt wider. Aufgrund der kleinen Fallzahl und der vermutlich stärkeren Resonanz von HÄ mit Interesse an Forschungsfragen und evidenzbasierter Medizin (Selektionsbias) ist diese Studie sicherlich nicht repräsentativ.

Patientenrekrutierung für Studien durch niedergelassene Ärzte ist ein schwieriges Problem der primärmedizinischen Versorgungsforschung. Das Interesse und das Engagement der $\mathrm{HÄ}$ für die Projekte sind wichtig; deren Zeitdruck und Vergesslichkeit bezüglich des Ablaufs der Studien sind die Hauptfaktoren, welche maximalen Rekrutierungszahlen im Wege stehen (Bell-Syer, Klaber Moffett 2000). In unserer Studie gaben 17 von 35 der angeschriebenen Allgemeinmediziner ihr Einverständnis zur Teilnahme (response rate: 49\%). Als Ablehnungsgründe wurden 
Zeitmangel, Unzumutbarkeit für die Patienten und Störung des Praxisablaufs genannt.

Die Datenerhebung in den hausärztlichen Praxen mittels eines FB für Patienten wählte man aus zwei Gründen. Zum einen bedeutete diese Methode für die teilnehmenden Allgemeinmediziner keinen zusätzlichen Arbeitsaufwand wie z.B. Freistellen eines Computers für EDV-Recherche oder Zeitaufwand für ein Interview bzw. einen FB. Zum anderen versprach das direkte Ansprechen von betroffenen Patienten mittels Poster im Wartezimmer bzw. Handzettel an der Anmeldung und das Ausfüllen des anonymen FB eine größere Teilnehmerzahl an der Studie. Ein Nachteil dieser Vorgehensweise ist ein hoher Selektionseffekt seitens der Patienten, da sich vermutlich nur „studienfreudige“ Patienten mit eher rezidivierenden oder chronischen KS zur Teilnahme an der Studie bereiterklärten, die sich gegebenenfalls Heilung durch die Mitarbeit erhofften. Dies könnten vor allem Patienten mit verstärktem Krankheitserleben und einer hohen Inanspruchnahme von rückenbezogenen Gesundheitsleistungen sein. Es wurde versucht, die Arzthelferinnen zu animieren, die Patienten direkt auf die Studie aufmerksam zu machen und zur Teilnahme zu motivieren, um eine möglichst konsekutive Rekrutierung zu gewährleisten. Dem widerspricht aber eine Responsrate von 64,5\%, die auf Basis der von den HÄ geführten Strichliste zu ermitteln ist.

Offene Fragen (Freitext) gaben Möglichkeit für individuelle Anmerkungen, die für die Studie von besonderer Wichtigkeit waren und zudem qualitative Informationen enthielten. Es mag jedoch sein, dass die Antworten der Patienten erwünschtes Verhalten widerspiegeln, indem sie z.B. bereits über die Bedeutung aktivierender Maßnahmen aufgeklärt wurden und den Wunsch nach Spritzen oder Massagen nicht äußerten. Durch die anonyme Form der Fragenbeantwortung konnte keine Korrektur fehlender Werte im Datensatz durch z.B. Rücksprache mit den Patienten vorgenommen werden (Dokumentationsbias).

Die Strichlisten von Arzthelferinnen und HÄ wurden in einem Abschlussgespräch bei fast allen teilnehmenden Praxisteams als nicht störend oder zeitaufwendig und teilweise sogar als abwechslungsreich beschrieben.

Patienten, die sich mit einem anschließendem Telefoninterview einverstanden zeigten, wurden 2 bzw. 12 Wochen nach der hausärztlichen Konsultation kontaktiert und zum Verlauf ihrer Beschwerden befragt. Dies erwies sich von der Handhabung 
her erneut als relativ einfach und unproblematisch. Kritisch zu betrachten ist jedoch das unterschiedliche Erinnerungsvermögen der einzelnen Patienten an die Abläufe der vorangegangenen Wochen. So werden besonders schmerzgeplagte Patienten oder Patienten, die sich invasiven Maßnahmen unterzogen (Spritzen etc.) besser an die Konsultation erinnern können, als Patienten, die „nur“ ein Rezept erhielten und entsprechend aufgeklärt wurden (Erinnerungsbias). Der Fehler konnte durch den Einsatz von Schmerz- oder Kostentagebüchern minimiert werden. In dieser Studie bot das Ausfüllen der Schmerztagebücher durch einige teilnahmewillige Patienten die Gelegenheit, Schmerzcharakteristika über eine weitere Woche (13. bzw. 14. Woche des Follow-up) zu beobachten und in die Auswertung einzubeziehen. Underwood et al. (1999) kritisieren, dass die meisten derzeit existierenden Maßeinheiten für KS und Funktionseinschränkungen lediglich eine Beurteilung über die momentanen Schmerzen und Einschränkungen erlauben. Angebrachter erscheint die Messung dieser Parameter (z.B. mit dem modifizierten Roland-MorrisFragebogen oder der von Korff-Skala) über einen vorausgehenden 4-WochenZeitraum. Diese definierte Periode zeigt ein umfangreicheres Bild über den Patientenstatus als eine Beurteilung des gleichen an nur einem Tag. In der vorliegenden Erhebung mit Tagebüchern war allerdings mit einem hohen Selektionseffekt seitens der Patienten zu rechnen.

Trotz der aufgeführten Fehlermöglichkeiten liefert die Methode unserer Studie interessante Informationen, die Ausgangspunkt für weitere Untersuchungen an höheren Patientenfallzahlen sein können.

\subsection{Ausblick und Schlussfolgerung}

Der HA ist als primärer Ansprechpartner bei der Versorgung von Patienten mit KS nicht mehr wegzudenken. Wie unsere Studie zeigt, konsultiert fast jeder 12. Patient einer Allgemeinarztpraxis seinen HA wegen KS. Aktuelle Leitlinien belegen, dass schon mit geringem Zeitaufwand, gründlicher Anamnese und einfachen noninvasiven Tests, eine klinische Unterscheidung zwischen unkomplizierten, radikulären und komplizierten $\mathrm{KS}$ möglich ist. Hierfür müssen Patienten keine aufwendigen, invasiven und vor allem teuren Verfahren durchlaufen. Nach dem frühzeitigen Erkennen bzw. dem Ausschluss gefährlicher Verläufe (Tumor, Fraktur, 
Trauma etc.), können Patienten mit dem HA eine adäquate Therapie erarbeiten (Beratungsgespräch) und umsetzen. Da über $70 \%$ der Patienten die Behandlung ihrer Schmerzen als wichtigstes Ziel bei der hausärztlichen Konsultation ansehen, sollte eine effektive Analgesie im Vordergrund der Therapie stehen. Daneben suchen Patienten vor allem Aufklärung und Beratung. Dieses Ergebnis zeigt den Bedarf an hausärztlichen Gesprächsführungskompetenzen und in Hinblick auf den aktivierenden Therapieansatz den Bedarf an motivierender Beratung. Ein großer Anteil der hausärztlichen Kreuzschmerzpatienten leidet unter chronischen Beschwerden. Hierfür haben sich multimodale Therapiekonzepte als effektiv erwiesen. Therapieplätze bzw. entsprechende Netzwerke sind in Deutschland jedoch selten. Alternativ kann auf ambulante oder stationäre Rehabilitation ausgewichen werden, aber auch die ist nur einem Teil der Patienten mit langen Wartezeiten zugänglich.

Aufgrund der kleinen Fallzahl insbesondere bei den Telefoninterviews und den Schmerztagebüchern müssen die Ergebnisse dieser Studie mit Vorbehalt betrachtet werden. Weitere Untersuchungen - auch qualitativer Art - sind notwendig, um die Leitlinienadhärenz von $\mathrm{HÄ}$, die wesentlich auch auf der Interaktion von Patienten und Ärzten beruht, besser zu verstehen und die Voraussetzungen für mögliche qualitätssichernde Maßnahmen zu schaffen. 


\section{Zusammenfassung}

Hintergrund/ Ziel: Das klinische Wissen über das Auftreten und den Verlauf von KS in hausärztlichen Praxen ist bisher begrenzt. Für die Implementierung evidenzbasierter Leitlinien ist die Kenntnis der hausärztlichen Versorgungssituation unentbehrlich. In einer Querschnittserhebung mit anschließender prospektiver Kohortenstudie sollen Epidemiologie, Krankheitsverlauf, Behandlung sowie Krankheitsvorstellungen und Erwartungen von Kreuzschmerzpatienten in Allgemeinarztpraxen untersucht werden.

Methode: Patienten mit KS wurden vor einer HA-Konsultation in der Praxis schriftlich zu ihren Beschwerden befragt. In zwei Telefoninterviews (nach 2 und 12 Wochen) wurden der Schmerzverlauf und die stattgefundenen Versorgungsleistungen erfasst.

Ergebnisse: 17 von 35 angeschriebenen HÄ (49\%) im Bezirk Südoldenburg nahmen an der Studie teil. 127 Patienten beteiligten sich an der schriftlichen Befragung, 49 am Telefoninterview nach 2 Wochen und 40 nach 12 Wochen. Die mittlere 1Wochen-Prävalenz von Kreuzschmerzpatienten in der hausärztlichen Praxis betrug 7,9\%, die Inzidenz 5,5\%. An Rezidiven litten 19\% der Patienten. Nach 12 Wochen klagten trotz Therapie noch $55 \%$ unter fast täglichen bis häufigen Kreuzschmerzepisoden. Über die Hälfte (54\%) der teilnehmenden Patienten versuchten bereits vor der Konsultation ihre Schmerzen mit Bewegung, Wärmeanwendungen und Schmerztabletten in den Griff zu bekommen. Vom Arzt wünschten sie sich die Kontrolle ihrer Schmerzen (23\% hielten Spritzen für sinnvoll), weniger Diagnostik. In der ärztlichen Behandlung wurden NSAR sowie physikalische und physiotherapeutische Maßnahmen eingesetzt. 28,5\% von 49 Patienten erhielten intramuskuläre Injektionen. Überweisungen erfolgten zu Orthopäden und Neurologen, nicht jedoch zu Psychotherapeuten. 62,5\% von 40 Patienten wurden zu aktiver Bewegung angeregt, Bettruhe empfahl kein HA.

Schlussfolgerung: Zwar scheint ein Großteil der Patienten und Ärzte über die Bedeutung eines aktivierenden Behandlungsansatzes in der Therapie von KS informiert zu sein, doch werden trotzdem in hausärztlichen Praxen nicht evidenzbasierte und obsolete Therapieverfahren eingesetzt. Dies mag zum Teil auf die Erwartungen und Wünsche der Patienten zurückzuführen sein. 


\section{Literaturverzeichnis}

Ahrens S: Psychosomatik chronischer Schmerzsyndrome. In: Wards R. (Hrsg.). Chronischer Schmerz und Psyche. Fischer Verlag, Stuttgart 1990, 1-9

Becker A, Chenot JF, Niebling W, Kochen MM: DEGAM Leitlinie Kreuzschmerzen. Omikron Publishing, Düsseldorf 2003

Bell-Syer SEM, Klaber Moffett JA (2000): Recruiting patients to randomized trials in primary care: principles and case study. Fam Pract 17, 187-91

Bendix AF, Bendix T, Ostenfeld S, Bush E, Andersen A (1995): Active treatment programs for patients with chronic low back pain: a prospective, randomized, observer-blinded study. Eur Spine J $\underline{4}, 148-52$

Berger-Schmitt R, Kohlmann T, Raspe H (1996): Rückenschmerzen in Ost- und Westdeutschland. Gesundheitswesen $\underline{58}, 519-24$

Biering-Soerensen $F(1983)$ : A prospective study of low back pain in a general population. Scand J Rehabil Med $\underline{15}$, 71-9

Breitenfelder J (1988): Der Kreuzschmerz - Ein interdisziplinäres Problem. Krankenhausarzt $\underline{61}, 5$

Buchner M, Neubauer E, Barie A, Schiltenwolf M (2007): Komorbidität bei Patienten mit chronischen Rückenschmerzen. (Schmerz, im Druck)

Car J, Sheikh A (2003): Acute low back pain. BMJ 327, 541

Cherkin DC, Sherman KJ, Deyo RA, Shekelle PG (2003): A review of the evidence for the effectiveness, safety, and cost of acupuncture, massage therapy, and spinal manipulation for back pain. Ann Intern Med 138, 898-906

Clarke J, van Tulder M, Blomberg S, de Vet H, van der Heijden, Bronfort G (2006): Traction for Low Back Pain With or Without Sciatica: An updated systematic review within the framework of the Cochrane Collaboration. Spine $\underline{31}, 1591-99$

Clinical Standards Advisory Group. Epidemiology Review: The epidemiology and cost of back pain. Annex to the CSAG report on back pain. HMSO, London 1994 (review of epidemiological studies)

Cockburn J, Pit S (1997): Prescribing behaviour in clinical practice: patients' expectations and doctors' perceptions of patients' expectations - a questionnaire study. BMJ $\underline{315}, 520-3$

Croft PR, Joseph S, Cosgrove S, Jordan L, Papageorgiou A, Pope D, Ferry S, Jayson MIV, Silman A: Low back pain in the community and in hospitals. A report to the Clinical Standards Advisory Group of the Department of Health. HMSO, London 1994 
Croft PR, Papageorgiou A, McNally R (1997): Low back pain. In: Waddell G. The back pain revolution. $1^{\text {st }}$ Edition; Churchill-Livingstone, Edinburgh 1998, 73

Croft PR, Macfarlane GJ, Papageorgiou AC, Thomas E, Silman AJ (1998): Outcome of low back pain in general practice: a prospective study. BMJ $\underline{316}, 1356-9$

Deyo RA (1993): Newer thinking on diagnosis and therapy. Consultant $\underline{33}, 88-100$

Deyo RA, Weinstein DO (2001): Low back pain. N Engl J Med $\underline{344}$, 363-70

Eisenberg DM, Davis RB, Ettner SL, Appel S, Wilkey S, van Rompay M, Kessler RC (1998): Trends in alternative medicine use in the United States, 1990-1997: results of a follow-up national survey. JAMA $\underline{280}, 1569-1575$

Frankel BSM, Moffett JK, Keen S, Jackson D (1999): Guidelines for low back pain: changes in GP management. Fam Pract $\underline{16}, 216-22$

Freeborn DK, Shye D, Mullooly JP, Eraker S, Romeo J (1997): Primary care physicians' use of lumbar spine imaging tests. J Gen Intern Med 12, 619-25

Frost H, Lamb SE, Doll HA, Carver PT, Stewart S (2004): Randomised controlled trial of physiotherapy compared with advice for low back pain. BMJ $\underline{329}, 708$

Frymoyer JW, Pope MH, Constanza MC, Rosen, JC, Goggin JE, Wilder DG (1980): Epidemiologic studies of low back pain. Spine $\underline{5}, 419-23$

Frymoyer JW, Cats-Baril WL (1991): An overview of the incidences and costs of low back pain. Orthop Clin North Am 22, 263-71

Furlan AD, Brosseau L, Welch V, Wong J: Massage for low back pain; in: The Cochrane Library, Issue 4. John Wiley and Sons, Ltd. Chichester, 2000

Furlan AD, van Tulder MW, Cherkin D, Tsukayama H, Lao L, Koes BW, Berman B (2005): Acupuncture and dry-needling for low back pain: an updated systematic review within the framework of the cochrane collaboration. Spine $\underline{30}, 944-63$

Göbel H (2001): Epidemiologie und Kosten chronischer Schmerzen: Spezifische und unspezifische Rückenschmerzen. Schmerz $\underline{15}, 92-8$

Hagen KB, Jamtvedt G, Hilde G, Winnem M (2005): Bed rest for acute low back pain and sciatica. Spine $\underline{30}, 542-6$

Hasenbring M: Chronifizierung bandscheibenbedingter Schmerzen. Schattauer, Stuttgart 1992

Hayden JA, van Tulder MW, Malmivaara A, Koes BW: Exercise therapy for treatment of non-specific low back pain. The Cochrane Library, Issue 3. John Wiley and Sons, Ltd. Chichester, 2005

Heliovaara M, Impivaara O, Sievers K, Melkas T, Knekt P, Korpi J, Aromaa A (1987): Lumbar disc syndrome in Finland. J Epidemiol Community Health $\underline{41}, 251-8$ 
Henley $E$ (2000): Understanding and treating low back pain in family practice. Fam Pract $\underline{49}, 793-95$

Heymans MW, van Tulder MW, Esmail R, Bombardier C, Koes BW: Back schools for non-specific low back pain. The Cochrane Library, Issue 4. John Wiley and Sons, Ltd. Chichester, 2004

Hildebrandt VH, Proper KI, van den Berg R, Douwes M, van den Heuvel SG, van Buuren S (2000): Cesar therapy is temporarly more effective in patients with chronic low back pain than standard treatment by family practioner: randomized, controlled and blinded clinical trial with one year follow-up. Ned Tijdschr Geneeskd 144, 225864

Keel P, Schütz-Petitjean D: Einleitung. In: Keel P, Schütz-Petitjean D (eds). Chronifizierung von Rückenschmerzen: Hintergründe, Auswege. Eular Verlag, Basel 1996, 11-30

Keen S, Dowell AC, Hurst K, Klaber Moffett JA, Tovey P, Williams R (1999): Individuals with low back pain: how do they view physical activity? Fam Pract $\underline{16}$, 3945

Keller S: Motivation zur Verhaltensänderung - Das Transtheoretische Modell in Forschung und Praxis. Lambertus, Freiburg 1999

Khadilkar A, Milne S, Brosseau L, Wells G, Tugwell P, Robinson V, Shea B, Saginur M (2005): Transcutaneous electrical nerve stimulation (TENS) for chronic low back pain. Spine $\underline{30}, 2657-66$

Kochen MM (1999): Erwartungen von Patienten an die ärztliche Ausbildung. Z Ärztl Fortb Qualitätssich $\underline{93}$, 599-604

Koes BW, Scholten RJ, Mens JM, Bouter LM (1997): Efficacy of non-steroidal antiinflammatory drugs for low back pain: a systematic review of randomised clinical trials. Ann Rheum Dis $\underline{56}, 214-23$

Koes BW, van Tulder MW, Ostelo R, Kim Burton A, Waddell G (2001): Clinical guidelines for the management of low back pain in primary care: an international comparison. Spine 26, 2504-13

Mason V: The prevalence of back pain in Great Britain. Office of Population Censuses and Surveys, Social Survey Division. HMSO, London 1994, 1-24

McKinley RK, Middleton JF (1999): What do patients want from doctors? Content analysis of written patient agendas for the consultation. $\mathrm{Br} \mathrm{J}$ Gen Pract $\underline{49}, 796-800$

Nachemson A, Bigos SJ: The low back. In: Cruess J, Rennie WRJ, editors. Adult Orthopedics. Churchill-Livingstone, New York 1984, 843-937

Negrini S, Politano E, Carabalona R, Mambrini A (2001): General practioners' management of low back pain: impact of clinical guidelines in a non-English-speaking country. Spine 26 , 2727-33 
Nelemans PJ, de Bie RA, de Vet HCW, Sturmans F: Injection therapy for subacute and chronic benign low back pain. The Cochrane Library, Issue 4. John Wiley and Sons, Ltd. Chichester, 1999

Nyiendo J, Haas M, Goodwin P (2000): Patient characteristics, practice activities and one-month outcomes for chronic, recurrent low back pain treated by chiropractors and family medicine physicians: a practice-based feasibility study. J Manipulative Physiol Ther $\underline{23}, 239-45$

Ostelo RWJG, van Tulder MW, Vlaeyen JWS, Linton SJ, Morley SJ, Assendelft WJJ: Behaviorial treatment for chronic low back pain. The Cochrane Library, Issue 1. John Wiley and Sons, Ltd. Chichester, 2004

Papageorgiou AC, Croft PR, Ferry S, Jayson MIV, Silman AJ (1995): Estimating the prevalence of low back pain in the general population. Evidence from the South Manchester back pain survey. Spine 20, 1889-94

Pengel LHM, Herbert RD, Maher CG, Refshauge KM (2003): Acute low back pain: systematic review of its prognosis. BMJ $\underline{327}, 323-8$

Pfingsten M, Hildebrandt J (2001): Treatment of chronic low back pain through intensive activation - an assessment of 10 years. Anasthesiol Intensivmed Notfall Schmerzther $\underline{36}, 580-9$

Prochaska JO, Velicer WF (1997): The Transtheoretical model of health behaviour change. Am J Health Promot 12, 38-48

Raspe HH, Kohlmann T (1993): Rückenschmerzen - eine Epidemie unserer Tage? Dtsch Ärztebl 44, 2920-5

Raspe HH, Kohlmann T (1994): Die aktuelle Rückenschmerzepidemie. Ther Umsch $\underline{51}, 367-74$

Raspe HH, Wasmus A, Greif G, Kohlmann T, Kindel P, Mahrenholz M (1990): Rückenschmerzen in Hannover. Akt Rheumat $\underline{15}$, 32-37

Rogers WA (2002): Whose autonomy? Which choice? A study of GP's attitudes towards patient autonomy in the management of low back pain. Fam Pract $\underline{19}$, 140-5

Sachverständigenrat zur Begutachtung der Entwicklung im Gesundheitswesen. Bedarfsgerechtigkeit und Wirtschaftlichkeit: Band III. 3: Ausgewählte Erkrankungen: Rückenleiden, Krebserkrankungen und depressive Störungen. Bundesministerium für Gesundheit. Baden-Baden 2002

Schers H, Braspenning J, Drijver R, Wensing M, Grol R (2000): Low back pain in general practice: reported management and reasons for not adhering to the guidelines in the Netherlands. Br J Gen Pract $\underline{50}$, 640-44

Schiottz-Christensen B, Nielsen GL, Hansen VK, Schodt T, Sorensen HAT, Olesen F (1999): Long-term prognosis of acute low back pain in patients seen in general practice: a 1 year prospective follow-up study. Fam Pract $\underline{16}, 223-32$ 
Schipperges H: Erwartungen an den Arzt von morgen - Medizin und Grenzgebiete, Perspektiven für die 90'er Jahre (Medicenale XX Band 2, 2. Auflage). Medice Hausdruck, Iserlohn 1990, 1095-1110

Schlemmer J: Was der Patient von seinem Arzt zu erhoffen wagt - Medizin und Grenzgebiete, Perspektiven für die 90'er Jahre (Medicenale XX Band 2, 2. Auflage). Medice Hausdruck, Iserlohn 1990, 1111-8

Schochat T, Jackel WH (1998): Prevalence of low back pain in the population. Rehabilitation $\underline{37}, 216-23$

Skelton AM, Murphy EA, Murphy RJL, O'Dowd TC (1995): General practitioner perceptions of low back pain patients. Fam Pract 12, 44-8

Skelton AM, Murphy EA, Murphy RJL, O'Dowd TC (1996): Patients' views of low back pain and its management in gerneral. Br J Gen Pract $\underline{46}$, 153-6

Skovron ML, Szpalski M, Nordin M, Melot C, Cukier D (1994): Sociocultural factors and back pain. A population-based study in Belgian adults. Spine $\underline{19}, 129-137$

Southgate LJ, Bass MJ (1983): Determination of worries and expectations of family practice patients. J Fam Pract $\underline{16}$, 339-44

Stahmann S: Das Profil von Rückenschmerzen in einer ländlichen Allgemeinarztpraxis. Med. Diss. Lübeck 2001

Staiger TO, Jarvik JG, Deyo RA, Martin B, Braddock CH $3^{\text {rd }}$ (2005): Brief report: Patient-physician agreement as a predictor of outcomes in patients with back pain. $\mathrm{J}$ Gen Intern Med. 10, 935-7

Underwood MR, Barnett AG, Vickers MR (1999): Evaluation of two time-specific back pain: outcome measures. Spine $\underline{24}, 1104-12$

van den Hoogen HJ, Koes BW, van Eijk JT, Bouter LM, Deville W (1998): On the course of low back pain in general practice: a one year follow up study. Ann Rheum Dis $\underline{57}, 13-9$

van Tulder MW, Touray T. Furlan AD, Solway S. Bouter LM: Muscle relaxants for non-specific low back pain. The Cochrane Library, Issue 4. John Wiley and Sons Ltd. Chichester, 2003

van Tulder MW, Koes BW, Malmivaara A (2006):Outcome of non-invasive treatment modalities on back pain: an evidence-based review. Eur Spine J 1, 64-81

Waddell G (1982): An approach to backache. Br J Hosp Med 23,187-219

Waddell G (1987): A new clinical model for the treatment of low back pain. Spine $\underline{12}$, $632-44$

Waddell G (Ed.): The back pain revolution. $1^{\text {st }}$ Edition; Churchill-Livingstone, Edinburgh 1998 
Waddell G, Burton AK (2005): Concepts of rehabilitation for the management of low back pain. Best Pract Res Clin Rheumatol $\underline{4}$, 655-70

Waddell D, Feder G, Lewis M (1997): Systematic reviews of bedrest and advice to stay active for acute low back pain. Br J Gen Prac $\underline{47}, 647-52$

Walsh K, Cruddas M, Coggon D (1992): Low back pain in eight areas of Britain. J Epidemiol Community Health $\underline{46}, 227-230$

Waxman R, Tennant A, Helliwell P (1998): Community survey of factors associated with consultation for low back pain. BMJ $\underline{317}, 1564-7$

White AA, Gordon SL (1982): Synopsis: workshop on idiopathic low back pain. Spine $\underline{7}, 141-9$

Wörz R, Müller-Schwefe G, Stroehmann I, Zeuner L, Zieglgänsberger W, Zimmermann M (2000): Rückenschmerzen: Leitlinien der medikamentösen Therapie. Sonderdruck. MMW 142, 27-33 


\section{Abkürzungsverzeichnis}

\begin{tabular}{ll} 
AU & Arbeitsunfähigkeit \\
bzw. & beziehungsweise \\
ca. & circa \\
DEGAM & Deutsche Gesellschaft für Allgemeinmedizin und Familienmedizin \\
EDV & Elektronische Datenverarbeitung \\
etc. & et cetera \\
evtl. & eventuell \\
FB & Fragebogen \\
ggf. & gegebenenfalls \\
HA/HÄ & Hausarzt(in)/ Hausärzte(innen) \\
insg. & insgesamt \\
KS & Kreuzschmerzen \\
NSAR & Nicht steroidale Antirheumatika \\
o.g. & oben genannt \\
Prof. & Professor \\
RCT/ RCTs & Randomised Controlled Trial/s \\
S. & siehe \\
TENS & Transkutane elektrische Nervenstimulation \\
USA & United States of America \\
z.B. & zum Beispiel \\
\hline
\end{tabular}


9. Anhang

9.1 Handzettel für den Arzttresen

Liebe Patientin, lieber Patient,

\section{haben Sie heute Kreuzschmerzen?}

Auch wenn heute sehr viel über Ursachen und Behandlung von Kreuzschmerzen bekannt ist, wissen wir wenig darüber.

- wie Patienten ihre Beschwerden erleben

- und was Sie sich von ihrer Behandlung erhoffen.

Die Universität Göttingen führt zu diesen Fragen eine Studie in unserer Praxis durch.

Sollten Sie heute Kreuzschmerzen haben, möchten wir Sie bitten, einen kleinen Fragebogen auszufüllen. Er liegt an der Anmeldung für Sie bereit .

\section{Vielen Dank!}

Verantwortlich: Dr. A. Becker, Abt. Allgemeinmedizin, Universität Göttingen 


\subsection{Zettel für die Anmeldung bzw. Strichliste für den Hausarzt}

a) Zettel für die Arzthelferinnen an der Anmeldung:

„Bitte führen Sie eine Liste aller Patienten, die in dieser Woche die Praxis aufsuchen, unabhängig vom Grund ihres Besuches!"

\begin{tabular}{|l|l|}
\hline & Anzahl der Patienten \\
\hline Montag & \\
\hline Dienstag & \\
\hline Mittwoch & \\
\hline Donnerstag & \\
\hline Freitag & \\
\hline Gesamt: & \\
\hline
\end{tabular}

b) Zettel für den Hausarzt:

„Bitte führen Sie eine Liste aller Patienten, die Sie aufgesucht haben und vermerken diejenigen Patienten, die Sie in dieser Woche mit Kreuzschmerzen konsultieren! “

\begin{tabular}{|l|l|l|}
\hline & $\begin{array}{c}\text { Anzahl der Patienten } \\
\text { insgesamt }\end{array}$ & Anzahl der Kreuzschmerzpatienten \\
\hline Montag & & \\
\hline Dienstag & & \\
\hline Mittwoch & & \\
\hline Donnerstag & & \\
\hline Freitag & & \\
\hline Gesamt: & & \\
\hline
\end{tabular}




\subsection{Fragebogen}

\section{Kreuzschmerzen in der Hausarztpraxis}

1. Kreuzschmerzen sind mal stärker, mal schwächer. Oft dauern sie mehrere Tage oder Wochen an.

Haben Sie das erste Mal in Ihrem Leben Kreuzschmerzen?

$$
\square \mathrm{Ja} \quad \square \text { Nein }
$$

Wenn nein, wann hatten Sie das letzte Mal Kreuzschmerzen?

$\square$ vor weniger als einem Monat $\quad \square$ vor 1-6 Monaten $\quad \square$ vor mehr als 6 Monaten

2. Wie stark waren Ihre Schmerzen durchschnittlich in den letzten $\mathbf{2 4}$ Stunden?

(O bedeutet keine Schmerzen, 10 sind die stärksten Schmerzen, die Sie sich vorstellen können)

$$
\begin{aligned}
& \text { (1) - (1) - (2) - (3) - (4) - (5) - (6) - (7) - (8) - (9) - (10) } \\
& \text { Keine Schmerzen }
\end{aligned}
$$

3. Haben Sie selbst schon etwas versucht, um Ihre jetzigen Schmerzen in den Griff zu bekommen?

$\square$ Nein Ja, nämlich: $\square$ Schmerztablette $\quad \square$ Wärmflasche

$\square$ Bettruhe $\quad \square$ Bewegung (z.B. Gymnastik, Schwimmen)

Sonstiges:

4. Was ist Ihrer Meinung nach zur Zeit die beste Behandlung für Ihre Beschwerden? (Bitte wählen Sie nur eine zutreffende Antwort aus!)
$\square$ Bettruhe
$\square$ Tablette gegen die Schmerzen
$\square$ Krankengymnastik
$\square$ Einrenken
$\square$ Massagen
$\square$ eine Spritze gegen die Schmerzen
$\square$ Bewegung
$\square$ „Quaddeln“ (mehrere Injektionen in den schmerzenden Bereich)
Sonstiges: 
5. Sollten Ihrer Ansicht nach noch weitere Untersuchungen durchgeführt werden?

$\square$ Nein $\square$ Ja, welche?

6. Was ist für Sie bei Ihrem heutigen Hausarztbesuch am Wichtigsten? ( Bitte wählen Sie nur eine zutreffende Antwortmöglichkeit aus!)

$\square$ Behandlung der Schmerzen

$\square$ Tips \& Ratschläge für Zuhause

$\square$ Krankschreibung

$\square$ Weitere Diagnostik (Überweisung zum Orthopäden; Röntgen)

Sonstiges

7. Welche Fragen in Bezug auf Ihre Schmerzen möchten Sie Ihrem Arzt heute unbedingt stellen?

1.

2.

8. Würden Sie uns jetzt noch einige Angaben zu Ihrer Person machen?

Alter:

$\square$ weiblich $\quad \square$ männlich

Vielen Dank für das Ausfüllen des Fragebogens!

Bitte wenden! 


\subsection{Telefoninterview nach 2 und 12 Wochen}

Telefoninterview mit Patient

am 01

Einige kurze Fragen zu Ihrem Arztbesuch....

1. Sind Ihnen Medikamente verschrieben worden?

Wenn Ja, welche (auf Verpackung hinweisen)

Wie lange soll die Einnahme erfolgen?

2. Haben Sie eine Spritze bekommen?

$\square \mathrm{Ja}$

$\square$ Nein
Wenn Ja, welche?
$\square$ ins Gesäß
$\square$ in den Rücken
$\square$ Quaddeln

3. Welche weitere Behandlung wurde verordnet oder gemacht (am Tag der Konsultation)?

$\square$ Kurzwelle

$\square$ Krankengymnastik

$\square$ Einrenken

$\square$ Akupunktur

$\square$ Massage

4. Wurden noch weitere Tests gemacht, z.B. ein Röntgenbild?

$\square \mathrm{Ja}$,

5. Haben Sie eine Überweisung zu einem Facharzt erhalten?

$\square \mathrm{Ja}$, zum

6. Sind Ihnen vielleicht auch Tips und Anregungen für Zuhause mitgegeben worden?

Welche?

$\square$ Wärme $\quad \square$ auf Sitzposition achten

$\square$ Bettruhe $\quad \square$ Aktivität(welche?

7. Sind Sie bei Ihrem Arztbesuch wegen Rückenschmerzen krank geschrieben worden?

$\square$ Ja, seit wann?

$\square$ Nein

8. Haben Sie einen Rentenantrag gestellt?

$\square \mathrm{Ja}$

$\square$ Nein

9. Vor dem Besuch bei Ihrem Hausarzt hatten Sie ja gewisse Erwartungen.....

Was war für Sie am Wichtigsten bei diesem Arztbesuch(siehe Frage 6 im FB) ? Haben sich Ihre Erwartungen erfüllt? 
Jetzt würde ich Ihnen gern noch einige Fragen zu Ihrem Wohlbefinden in den letzten 24 Stunden stellen...

10. Wie stark waren Ihre Schmerzen durchschnittlich in den letzten 24 Stunden?

$$
\begin{aligned}
& \text { (1) - (1) - (2) - (3) - (4) - (5) - (6) - (7) - (8) - (9) - (10) } \\
& \text { maximal denkbare Schmerzen }
\end{aligned}
$$

11. Wurden Sie heute durch Ihre Schmerzen in Ihren Tätigkeiten und Bedürfnissen eingeschränkt?

$$
\begin{aligned}
& \text { (0) - (1) - (2) - (3) - (4) - (5) - (6) - (7) - (8) - (9) - (10) } \\
& \text { sehr stark beeinträchtigt }
\end{aligned}
$$

12. Haben Sie heute, außer durch Medikamente selbst Einfluss auf Ihre Schmerzen nehmen können?

$$
\begin{aligned}
& \text { (0) - (1) - (2) - (3) - (4) - (5) - (6) - (7) - (8) - (9) - (10) } \\
& \text { gar nicht } \quad \text { sehr gut }
\end{aligned}
$$

Wenn ja, wie?

13. Hat sich Ihre Beweglichkeit verbessert?

$\square \mathrm{Ja} \quad \square$ Nein

14. Wie viele Minuten waren Sie in den letzten 24 Stunden körperlich [im sportlichen Sinn] aktiv? (Gehen, Laufen, Radfahren, Schwimmen) [ $\rightarrow$ ggf. auch Hausarbeit, Beruf, Alltag]

15. Wie wohl haben Sie sich heute gefühlt?

$$
\begin{aligned}
& \text { (0) - (1) - (2) - (3) - (4) - (5) - (6) - (7) - (8) - (9) - (10) } \\
& \text { sehr schlecht }
\end{aligned}
$$

16. Glauben Sie, dass sich Ihre Kreuzschmerzen wieder bessern werden?
$\square$ eher ja
$\square$ eher nein

17. Was glauben Sie, ist die Ursache Ihrer Kreuzschmerzen?

18. Haben Sie den Eindruck, dass sich Ihre Stimmung auf Ihre Schmerzen auswirken könnte?

$\square \mathrm{Ja}$

Nein 


\subsection{Schmerztagebuch}

\section{Schmerztagebuch}

1. Tag

Datum:

\section{Alle Angaben beziehen sich auf die letzten 24 Stunden!}

Wie stark waren Ihre Schmerzen durchschnittlich in den letzten 24 Stunden?

(0) - (1) - (2) - (3) - (4) - (5) - (6) - (7) - (8) - (9) - (1)
keine Schmerzen

Haben Sie in den letzten 24 Stunden Schmerzmittel genommen? $\square$ Ja $\quad \square$ Nein

Wenn Ja, welche Medikamente oder Spritzen?

2 Haben Sie heute wegen Ihrer Schmerzen einen Arzt aufgesucht? $\square$ Ja $\square$ Nein

Wenn Ja, wen?

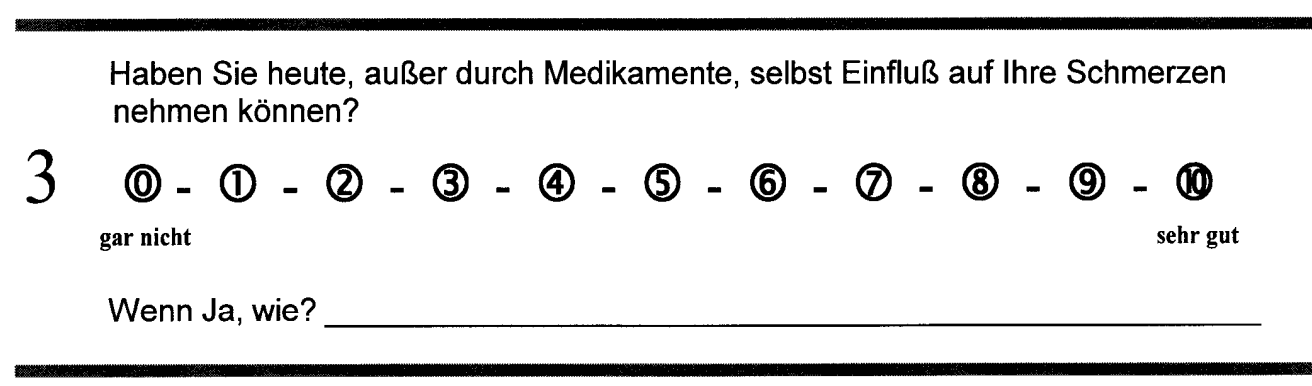

Wurden Sie heute durch Ihre Schmerzen in Ihren Tätigkeiten und Bedürfnissen eingeschränkt?

$$
4 \text { (1) - (1) - (2) - (3) - (4) - (5) - (6) - (7) - (8) - (9) - (1) }
$$

Wieviel Minuten waren Sie heute neben Ihren üblichen Aktivitäten sportlich aktiv (zügiges Gehen, Laufen, Radfahren, Schwimmen, Gartenarbeit usw.)?

5 Minuten, z.B.:

Wie wohl haben Sie sich heute gefühlt?
6 (0) - (1) -
(2) -
(3) - (4) - (5) -
(6) - (7) -
(8) - (9) - (1)
sehr gut




\section{Danksagung}

Für die freundliche Überlassung des Themas der Dissertation danke ich Herrn Prof. Dr. MM Kochen, MPH, FRCGP.

Mein besonderer Dank gilt Frau Prof. Dr. A. Becker, MPH, für die engagierte und hervorragende Betreuung meiner Arbeit.

Weiterhin möchte ich den Ärztinnen, Ärzten und Arzthelferinnen der teilnehmenden Arztpraxen für die Zusammenarbeit danken. 


\section{Lebenslauf}

Am 28.06.1976 wurde ich in Annaberg-Buchholz geboren.

Von 1982-1989 besuchte ich die Grund- und Gesamtschule in Oschatz. 1989 wechselte ich auf das allgemeine Gymnasium der Stadt Lohne, Niedersachsen. Nach dem 10. Schuljahr absolvierte ich von 1993-94 ein High-School-Jahr in Montgomery, Alabama, USA. Am 09.06.1997 erwarb ich in Lohne die allgemeine Hochschulreife.

Im Wintersemester 1997 nahm ich mein Studium der Humanmedizin an der Semmelweis Universität in Budapest, Ungarn auf. Ich beendete den vorklinischen Studienteil mit erfolgreichem Abschluss des Physikums am 25.08.1999. Den klinischen Teil des Medizinstudiums begann ich im Wintersemester 1999 an der Georg-August-Universität Göttingen. Nach Bestehen des ersten (29.08.2000) und zweiten (21.03.2003) Staatsexamens in Göttingen, absolvierte ich nach dem praktischen Jahr in Westerstede (Lehrkrankenhaus der Universität Göttingen) den dritten Abschnitt der ärztlichen Prüfung am 27.04.2004.

Seit dem 01.06.2004 bin ich als Assistenzärztin der Abteilung für Anästhesie, Intensiv- und Notfallmedizin in der Ammerlandklinik Westerstede, Niedersachsen tätig. 